\title{
Transition-Metal-Free Self-Hydrogen-Transferring Allylic Isomerization
}

\author{
Hong-Xing Zheng, ${ }^{\dagger}$ Zu-Feng Xiao, ${ }^{\dagger}$ Chuan-Zhi Yao, ${ }^{\dagger}$ Qiang-Qiang Li, ${ }^{\dagger}$ Xiao-Shan Ning, ${ }^{\dagger}$ \\ Yan-Biao Kang, ${ }^{*}, \dagger$ and Yong Tang ${ }^{\dagger, \dagger}$ \\ ${ }^{\dagger}$ Center of Advanced Nanocatalysis, Department of Chemistry, University of Science and Technology of China, Hefei, Anhui \\ 230026, China \\ \$The State Key Laboratory of Organometallic Chemistry, Shanghai Institute of Organic Chemistry, CAS, 345 Lingling Lu, \\ Shanghai 200032, China \\ E-mail: ybkang@ustc.edu.cn
}

\section{Contents}

1. General Information

S1

2. General Procedures

$\mathrm{S} 1-\mathrm{S} 16$

3. Isotopic Labelling Experiments and Kinetic Study

S16-S28

4. EPR experiments and radical trapping experiments

S28-S30

5. ICP-AES analysis

S30

6. References

S31-S32

7. NMR Spectra

S33-S81

\section{General information}

${ }^{1} \mathrm{H}$ NMR and ${ }^{13} \mathrm{C}$ NMR spectra were recorded on a Bruker 400 spectrometer; chemical shifts are reported in $\delta$ units relative to $\mathrm{CHCl}_{3}\left({ }^{1} \mathrm{H} \delta=7.26,{ }^{13} \mathrm{C} \delta=77.36\right)$ or TMS $\left({ }^{1} \mathrm{H} \delta=0.00,{ }^{13} \mathrm{C} \delta=0.00\right)$. ICPAES and elemental analysis were tested by the service at University of Science and Technology of China. Solvents were pre-dried over activated $4 \AA$ molecular sieves and heated to reflux over sodium (toluene, THF, octane) or calcium hydride $\left(\mathrm{CH}_{3} \mathrm{CN}\right.$, DMF, DME, 1,4-dioxane, DMSO) under an argon atmosphere and collected by distillation.

\section{General procedures}

\subsection{Isomerization of Allylic Alcohols (Table 1)}

1a $(0.5 \mathrm{mmol})$ was weighed directly into a Schlenk tube and dried under vacuum for $15 \mathrm{~min}$. Then solvent (toluene, $1 \mathrm{~mL}$ ) was added and stirred. Base and ligands were added subsequently and stirred at 
$90{ }^{\circ} \mathrm{C}$ for 2 hours. The reaction mixture was directly examined by ${ }^{1} \mathrm{H}$ NMR spectrometer to determine the conversion and selectivity using 1,4-dioxane $(10.7 \mu \mathrm{L})$ and 1,3-dimethoxybenzene $(22 \mu \mathrm{L})$ as internal standards.

\subsection{Screening bases (Table S1)}

1a $(0.5 \mathrm{mmol})$ was weighed directly into a Schlenk tube and dried under vacuum for $15 \mathrm{~min}$. Then toluene $(1 \mathrm{~mL})$ was added under stirring. A base ( ${ }^{t} \mathrm{BuONa}, \mathrm{KOH}, \mathrm{NaH}$, KHMDS, $\mathrm{LiHMDS},{ }^{n} \mathrm{BuLi}, \mathrm{K}_{2} \mathrm{CO}_{3}$, $\left.\mathrm{CsCO}_{3}, 20 \mathrm{~mol} \%\right), 1,10$-phenanthroline (20 mol \%) were added subsequently and stirred at $90{ }^{\circ} \mathrm{C}$ for 2 $\mathrm{h}$. The mixture was directly examined on ${ }^{1} \mathrm{H}$ NMR spectrometer to determine the conversion and selectivity by using 1,4-dioxane (10.7 $\mu \mathrm{L})$ and 1,3-dimethoxybenzene $(22 \mu \mathrm{L})$ as internal standards.

Table S1. Screening bases (Data listed in Table 1 were omitted.)

\begin{tabular}{|c|c|c|c|c|}
\hline entry & base [mol \%] & $\mathbf{3 a}[\mathrm{mol} \%]$ & $\mathbf{T}\left[{ }^{\circ} \mathrm{C}\right]$ & conv. $[\%]$ \\
\hline 1 & KOH (20) & 20 & 90 & 83 \\
\hline 2 & $\mathrm{NaH}(20)$ & 20 & 90 & 32 \\
\hline 3 & KHMDS (20) & 20 & 90 & 60 \\
\hline 4 & LiHMDS (20) & 20 & 90 & 42 \\
\hline 5 & ${ }^{n} \operatorname{BuLi}(20)$ & 20 & 90 & 49 \\
\hline 6 & ${ }^{t} \mathrm{BuONa}(20)$ & 20 & 90 & 97 \\
\hline \multirow[t]{2}{*}{7} & LiHMDS (200) & - & r.t. & trace \\
\hline & ${ }^{n} \mathrm{BuLi}(200)$ & - & r.t. & trace \\
\hline 8 & ${ }^{t} \mathrm{BuOK}(200)$ & - & r.t. & 58 \\
\hline 9 & $\mathrm{~K}_{2} \mathrm{CO}_{3}(20)$ & 20 & 90 & trace \\
\hline 10 & $\mathrm{CsCO}_{3}(20)$ & 20 & 90 & trace \\
\hline
\end{tabular}

\subsection{Screening additive (Table S2)}

1a $(0.5 \mathrm{mmol})$ was weighed directly into a Schlenk tube and dried under vacuum for $15 \mathrm{~min}$. Then solvent (toluene, $1 \mathrm{ml}$ ) was added and stirred. additive (phenanthroline, neocuprorine, TEMPOL, 2,2'bipyridine, NBS, BPO, NHPI, 2,6-di-tert-butylpyridine, Proton-Sponge, TMEDA $5 \mathrm{~mol} \%$ ), ${ }^{t} \mathrm{BuONa}$ (5 mol \%, $2.5 \mathrm{mg}$ ) were added subsequently and stirred at $90{ }^{\circ} \mathrm{C}$ for $2 \mathrm{~h}$. After finished, the mixture was directly examined on ${ }^{1} \mathrm{H}$ NMR spectrometer to determine the conversion and selectivity by using 1,4dioxane $(10.7 \mu \mathrm{L})$ and 1,3-dimethoxybenzene $(22 \mu \mathrm{L})$ as internal standards.

Table S2. Screening additive (Data listed in Table 1 were omitted.)

\begin{tabular}{ccc|ccc}
\hline entry & aditive & conv. [\%] & entry & aditive & conv. [\%] \\
\hline 1 & neocuprorine & 84 & 4 & NHPI (2-hydroxyisoindoline-1,3-dione) & 0
\end{tabular}




\begin{tabular}{ccc|ccc}
2 & TEMPOL & 15 & 5 & Proton Sponge & 24 \\
3 & NBS & 0 & 6 & TMEDA & 14 \\
\hline
\end{tabular}

\subsection{Screening solvents (Table S3)}

1 a $(0.5 \mathrm{mmol})$ was weighed directly into a Schlenk tube and dried under vacuum for $15 \mathrm{~min}$. Then solvent (toluene, THF, DME, 1,4-dioxane, $\mathrm{CH}_{3} \mathrm{CN}$, DMF, DMSO and octane, $1 \mathrm{~mL}$ ) was added and stirred. ${ }^{t} \mathrm{BuONa}(5 \mathrm{~mol} \%, 2.5 \mathrm{mg}), 1,10$-phenanthroline $(5 \mathrm{~mol} \%, 4.5 \mathrm{mg})$ were then added and stirred at 90 or $75{ }^{\circ} \mathrm{C}$ for $2 \mathrm{~h}$. After finished, the reaction mixture was directly examined on ${ }^{1} \mathrm{H}$ NMR spectrometer to determine the conversion and selectivity by using 1,4-dioxane $(10.7 \mu \mathrm{L})$ and 1,3-dimethoxybenzene $(22$ $\mu \mathrm{L})$ as internal standards.

\section{Table S3. Screening solvents}

\begin{tabular}{cccc}
\hline entry & solvent & $\mathrm{T}\left[{ }^{\circ} \mathrm{C}\right]$ & conv. $[\%]$ \\
\hline 1 & 1,4 -dioxane & 90 & 8 \\
2 & $\mathrm{THF}$ & 75 & 0 \\
3 & $\mathrm{CH}{ }_{3} \mathrm{CN}$ & 75 & 26 \\
4 & $\mathrm{DMF}$ & 90 & 0 \\
5 & $\mathrm{DMSO}$ & 90 & 74 \\
6 & DME & 90 & 0 \\
7 & octane & 90 & 64 \\
8 & toluene & 90 & 97 \\
\hline
\end{tabular}

\subsubsection{Preparation of starting materials 1}

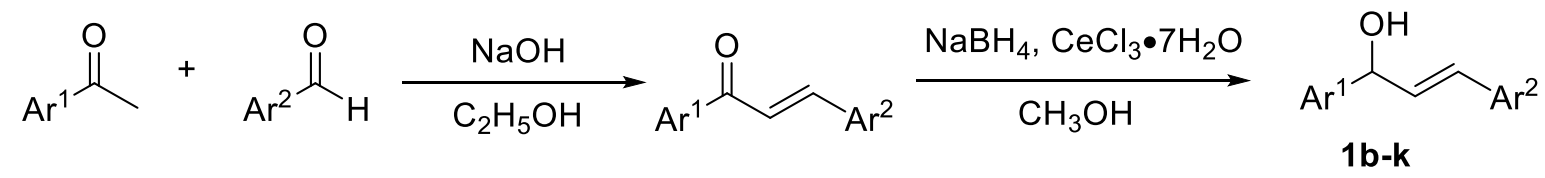

Compounds 1b-k were prepared as described in a reported protocol. ${ }^{1,2}$ General procedures: corresponding benzaldehyde $(10 \mathrm{mmol})$ was dissolved in ethanol $(20 \mathrm{~mL})$. Then aqueous $\mathrm{NaOH}(2.0$ equiv $)$ was added dropwise followed by the slow addition of the corresponding ketone $(10 \mathrm{mmol})$. The reaction mixture was stirred at room temperature until the complete consumption of the starting materials (monitored by TLC). The reaction mixture was diluted with water. The precipitate was collected by filtration and washed with water and EtOH. Allylic Alcohols were prepared by reduction of $\alpha, \beta$-unsaturated carbonyl compounds with $\mathrm{NaBH}_{4}$ or $\mathrm{LiAlH}_{4}$ in the presence of $\mathrm{CeCl}_{3}$. The reduction was quenched by addition of a mixed solution of saturated $\mathrm{NH}_{4} \mathrm{Cl}$ and $1 \mathrm{M} \mathrm{HCl}$ and extracted with $\mathrm{CH}_{2} \mathrm{Cl}_{2}$. The combined organic 
extracts were washed with brine, dried over anhydrous $\mathrm{MgSO}_{4}$, filtered, concentrated and purified on silica gel chromatography to give the allylic alcohols.

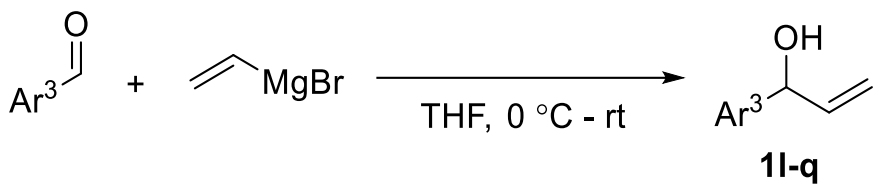

Compounds 11-q were prepared as described in a reported protocol. ${ }^{3}$ General procedure: vinyl magnesium bromide (2.0 equiv., 1.0 M in THF) was added slowly to a stirred solution of freshly distilled aldehyde (1.0 equiv) in dry THF $(0.5 \mathrm{M})$, which was maintained an internal temperature below $5^{\circ} \mathrm{C}$. After 15 min, the reaction was allowed to warm to r.t. and stirred for additional 1-3 $\mathrm{h}$. Then the reaction was quenched by adding saturated $\mathrm{NH}_{4} \mathrm{Cl}$ aq. and extracted with $\mathrm{Et}_{2} \mathrm{O}$. The combined organic extracts were washed with brine, dried over anhydrous $\mathrm{MgSO}_{4}$, filtered, concentrated and purified on silica gel chromatography (EtOAc/petroleum ether as eluent) to give the allylic alcohol.<smiles>OC(/C=C/c1ccc(F)cc1)c1ccccc1</smiles>

\section{(E)-3-(4-fluorophenyl)-1-phenylprop-2-en-1-ol (1b)}

Prepared according to the general procedure method $1 \mathrm{~h}$, white solid, $306 \mathrm{mg}, 68 \% .{ }^{1} \mathbf{H} \mathbf{~ N M R}(400 \mathrm{MHz}$, $\left.\mathrm{CDCl}_{3}\right) \delta 7.42-7.26(\mathrm{~m}, 7 \mathrm{H}), 7.00-6.94(\mathrm{~m}, 2 \mathrm{H}), 6.62(\mathrm{~d}, J=16.0 \mathrm{~Hz}, 1 \mathrm{H}), 6.28(\mathrm{dd}, J=16.0,6.4 \mathrm{~Hz}$, $1 \mathrm{H}), 5.34(\mathrm{~d}, J=5.6 \mathrm{~Hz}, 1 \mathrm{H}), 2.17(\mathrm{br} \mathrm{s}, 1 \mathrm{H}){ }^{4}$<smiles>CC=CC=CC(O)c1ccccc1</smiles>

\section{(E/Z)-1-phenylhexa-2,4-dien-1-ol (1c)}

Prepared according to the general procedure method $15 \mathrm{~min}$, yellow solid, $1.8 \mathrm{~g}, 70 \%$. ${ }^{1} \mathbf{H}$ NMR (400 $\left.\mathrm{MHz}_{\mathrm{CDCl}}\right) \delta: 7.40-7.28(\mathrm{~m}, 5 \mathrm{H}), 6.65(\mathrm{dd}, J=15.2,11.2 \mathrm{~Hz}, 0.12 \mathrm{H}), 6.27(\mathrm{dd}, J=15.2,10.4 \mathrm{~Hz}$, $0.87 \mathrm{H}), 6.09-5.99(\mathrm{~m}, 1 \mathrm{H}), 5.86(\mathrm{~d}, J=6.8 \mathrm{~Hz}, 0.23 \mathrm{H}), 5.79-5.70(\mathrm{~m}, 1.66 \mathrm{H}), 5.60-5.54(\mathrm{~m}, 0.14 \mathrm{H})$, $5.29(\mathrm{~d}, J=6.4 \mathrm{~Hz}, 0.13 \mathrm{H}), 5.23(\mathrm{~d}, J=5.6 \mathrm{~Hz}, 0.79 \mathrm{H}), 1.99(\mathrm{~d}, J=3.2 \mathrm{~Hz}, 0.13 \mathrm{H}), 1.95(\mathrm{~d}, J=3.2 \mathrm{~Hz}$, $0.84 \mathrm{H}), 1.83-1.79(\mathrm{~m}, 0.38 \mathrm{H}), 1.76(\mathrm{~d}, J=7.2 \mathrm{~Hz}, 2.62 \mathrm{H}) .^{5}$<smiles>OC(/C=C/c1ccccc1)c1ccc(Cl)cc1</smiles>

(E)-1-(4-chlorophenyl)-3-phenylprop-2-en-1-ol (1d) 
Prepared according to the general procedure method $10 \mathrm{~min}$, colorless oil, $574 \mathrm{mg}, 79 \% .{ }^{1} \mathbf{H}$ NMR (400 $\left.\mathrm{MHz}, \mathrm{CDCl}_{3}\right) \delta 7.37-7.23(\mathrm{~m}, 9 \mathrm{H}), 6.64(\mathrm{~d}, J=15.6 \mathrm{~Hz}, 1 \mathrm{H}), 6.30(\mathrm{dd}, J=15.6,6.6 \mathrm{~Hz}, 1 \mathrm{H}), 5.32(\mathrm{t}, J$ $=7.0 \mathrm{~Hz}, 1 \mathrm{H}), 2.21($ br s, $1 \mathrm{H}){ }^{6}$<smiles>OC(/C=C/c1ccccc1)c1cccc(Cl)c1</smiles>

\section{(E)-1-(3-chlorophenyl)-3-phenylprop-2-en-1-ol (1e)}

Prepared according to the general procedure method $1 \mathrm{~h}$, colorless oil, $2.3366 \mathrm{~g}, 96 \%$. ${ }^{1} \mathbf{H}$ NMR (400 $\left.\mathrm{MHz} \mathrm{CDCl}_{3}\right) \delta 7.45-7.25(\mathrm{~m}, 9 \mathrm{H}), 6.70(\mathrm{~d}, J=15.6 \mathrm{~Hz}, 1 \mathrm{H}), 6.34(\mathrm{dd}, J=15.6,6.8 \mathrm{~Hz}, 1 \mathrm{H}), 5.37(\mathrm{~d}, J$ $=6.8 \mathrm{~Hz}, 1 \mathrm{H}), 2.12-2.08(\mathrm{~m}, 1 \mathrm{H}){ }^{7}$<smiles>COc1ccc(C(O)/C=C/c2ccccc2)cc1</smiles>

\section{(E)-1-(4-methoxyphenyl)-3-phenylprop-2-en-1-ol (1f)}

Prepared according to the general procedure method $1 \mathrm{~h}$, colorless oil, $1.478 \mathrm{~g}, 61 \%$. ${ }^{1} \mathbf{H}$ NMR $(400 \mathrm{MHz}$, $\left.\mathrm{CDCl}_{3}\right) \delta 8.10-7.90(\mathrm{~m}, 6 \mathrm{H}), 7.63-7.58(\mathrm{~m}, 1 \mathrm{H}), 7.37(\mathrm{~d}, J=15.6 \mathrm{~Hz}, 2 \mathrm{H}), 7.09(\mathrm{dd}, J=15.6,6.4 \mathrm{~Hz}$, $1 \mathrm{H}), 6.04-6.03(\mathrm{~m}, 1 \mathrm{H}), 4.51(\mathrm{~s}, 3 \mathrm{H}), 2.90(\mathrm{br} \mathrm{s}, 1 \mathrm{H}){ }^{6}$<smiles>Cc1ccc(C(O)/C=C/c2ccccc2)cc1</smiles>

\section{(E)-3-phenyl-1-(p-tolyl)prop-2-en-1-ol (1g)}

Prepared according to the general procedure method $1 \mathrm{~h}$, white solid, $664 \mathrm{mg}, 98 \%$. ${ }^{1} \mathbf{H ~ N M R}(400 \mathrm{MHz}$, $\left.\mathrm{CDCl}_{3}\right) \delta 7.39-7.36(\mathrm{~m}, 2 \mathrm{H}), 7.32-7.27(\mathrm{~m}, 4 \mathrm{H}), 7.24-7.20(\mathrm{~m}, 1 \mathrm{H}), 7.17(\mathrm{~d}, J=8.4 \mathrm{~Hz}, 2 \mathrm{H}), 6.67(\mathrm{~d}, J$ $=16.0 \mathrm{~Hz}, 1 \mathrm{H}), 6.37(\mathrm{dd}, J=16.0,6.6 \mathrm{~Hz}, 1 \mathrm{H}), 5.34(\mathrm{dd}, J=6.0,2.8 \mathrm{~Hz}, 1 \mathrm{H}), 2.34(\mathrm{~s}, 3 \mathrm{H}), 2.04(\mathrm{~d}, J=$ $3.2 \mathrm{~Hz}, 1 \mathrm{H}) .^{8}$<smiles>Cc1ccc(/C=C/C(O)c2ccc(C)cc2)cc1</smiles>

\section{(E)-1,3-di-p-tolylprop-2-en-1-ol (1h)}

Prepared according to the general procedure method $15 \mathrm{~min}$, white solide, $636 \mathrm{mg}, 90 \% .{ }^{1} \mathbf{H}$ NMR (400 $\left.\mathrm{MHz}, \mathrm{CDCl}_{3}\right) \delta 7.29(\mathrm{dd}, J=15.6,8.0 \mathrm{~Hz} 4 \mathrm{H}), 7.17(\mathrm{~d}, J=8.0 \mathrm{~Hz}, 2 \mathrm{H}), 7.11(\mathrm{~d}, J=8.0,2 \mathrm{H}), 6.63(\mathrm{~d}, J$ $=16.0 \mathrm{~Hz}, 1 \mathrm{H}), 6.32(\mathrm{dd}, J=16.0,6.4 \mathrm{~Hz}, 1 \mathrm{H}), 5.33(\mathrm{~d}, J=6.4 \mathrm{~Hz}, 1 \mathrm{H}), 2.35(\mathrm{~s}, 3 \mathrm{H}), 2.32(\mathrm{~s}, 3 \mathrm{H}), 2.02$ $(\mathrm{d}, J=2.8 \mathrm{~Hz}, 1 \mathrm{H}) .{ }^{9}$ 
<smiles>Cc1ccc(C(O)/C=C/c2ccc(F)cc2)cc1</smiles>

(E)-3-(4-fluorophenyl)-1-(p-tolyl)prop-2-en-1-ol (1i)

Prepared according to the general procedure method $1 \mathrm{~h}$, white solid, $824 \mathrm{mg}, 45 \% .{ }^{1} \mathbf{H}$ NMR $(400 \mathrm{MHz}$, $\left.\mathrm{CDCl}_{3}\right) \delta 7.36-7.31(\mathrm{~m}, 4 \mathrm{H}), 7.19(\mathrm{~d}, J=8.0 \mathrm{~Hz}, 2 \mathrm{H}), 6.99(\mathrm{tt}, J=9.0,2.2 \mathrm{~Hz}, 2 \mathrm{H}), 6.64(\mathrm{~d}, J=15.6 \mathrm{~Hz}$, 1H), $6.30(\mathrm{dd}, J=16.0,6.4 \mathrm{~Hz}, 1 \mathrm{H}), 5.36-5.33(\mathrm{~m}, 1 \mathrm{H}), 2.36$ (s, 3H), 2.08-2.05 (m, 1H). ${ }^{13} \mathrm{C}$ NMR (100 $\left.\mathrm{MHz}, \mathrm{CDCl}_{3}\right) \delta 163.6,161.2,139.9,137.7,132.8(\mathrm{~d}), 131.5$ (d), 129.4, 129.1, 128.2, 128.1, 126.3, 115.6, 115.4, 74.9, 21.2. HRMS (ESI) calcd for $\mathrm{C}_{16} \mathrm{H}_{15} \mathrm{FONa}[\mathrm{M}+\mathrm{Na}]^{+}$265.1005, found 265.1009.<smiles>Cc1ccc(C(O)/C=C/c2ccc(C(F)(F)F)cc2)cc1</smiles>

(E)-1-(p-tolyl)-3-(4-(trifluoromethyl)phenyl)prop-2-en-1-ol (1j)

Prepared according to the general procedure method $1 \mathrm{~h}$, white solid, $709 \mathrm{mg}, 81 \%$. ${ }^{1} \mathbf{H ~ N M R}(400 \mathrm{MHz}$, $\left.\mathrm{CDCl}_{3}\right) \delta 7.55(\mathrm{~d}, J=8.0 \mathrm{~Hz}, 2 \mathrm{H}), 7.47(\mathrm{~d}, J=8.0 \mathrm{~Hz}, 2 \mathrm{H}), 7.32-7.30(\mathrm{~m}, 2 \mathrm{H}), 7.19(\mathrm{~d}, J=8.0 \mathrm{~Hz}, 2 \mathrm{H})$, $6.72(\mathrm{~d}, J=15.6 \mathrm{~Hz}, 1 \mathrm{H}), 6.47(\mathrm{dd}, J=15.6,5.6 \mathrm{~Hz}, 1 \mathrm{H}), 5.38(\mathrm{~d}, J=5.6 \mathrm{~Hz}, 1 \mathrm{H}), 2.36(\mathrm{~s}, 3 \mathrm{H}), 2.02$ (br s, 1H). ${ }^{13} \mathrm{C}$ NMR $\left(100 \mathrm{MHz}, \mathrm{CDCl}_{3}\right) \delta 140.3,139.6,138.0,134.4,129.8,129.6,129.4,128.7,126.9$, 126.5, 125.6 (q), 122.9, 74.8, 21.3. HRMS (EI) calcd for $\mathrm{C}_{17} \mathrm{H}_{15} \mathrm{~F}_{3} \mathrm{O}^{+}[\mathrm{M}]^{+}$292.1075, found 292.1081.<smiles>OC(/C=C/c1ccccn1)c1ccccc1</smiles>

\section{(E)-1-phenyl-3-(pyridin-2-yl)prop-2-en-1-ol (1k)}

Prepared according to the general procedure method $1 \mathrm{~h}$, yellow oil, $1.3153 \mathrm{~g}, 48 \% .{ }^{1} \mathbf{H}$ NMR $(400 \mathrm{MHz}$, $\left.\mathrm{CDCl}_{3}\right) \delta 8.49(\mathrm{dt}, J=4.8,0.8 \mathrm{~Hz}, 1 \mathrm{H}), 7.61(\mathrm{td}, J=7.6,2.0 \mathrm{~Hz}, 1 \mathrm{H}), 7.46-7.43(\mathrm{~m}, 2 \mathrm{H}), 7.38-7.34(\mathrm{~m}$, 2H), 7.32-7.27 (m, 2H), $7.12(\mathrm{ddd}, J=7.6,4.8,1.2 \mathrm{~Hz}, 1 \mathrm{H}), 6.89(\mathrm{dd}, J=16.0,5.6 \mathrm{~Hz}, 1 \mathrm{H}), 6.81(\mathrm{dd}, J$ $=16.0,0.8 \mathrm{~Hz}, 1 \mathrm{H}), 5.44(\mathrm{~d}, J=5.6 \mathrm{~Hz}, 1 \mathrm{H}), 3.32(\mathrm{~s}, 1 \mathrm{H}) .{ }^{13} \mathbf{C} \mathbf{N M R}\left(100 \mathrm{MHz}, \mathrm{CDCl}_{3}\right) \delta 155.2,149.4$, 142.5, 136.6, 136.5, 129.5, 128.6, 127.8, 126.5, 122.3, 121.8, 74.5. HRMS (ESI) calcd for $\mathrm{C}_{14} \mathrm{H}_{14} \mathrm{NO}$ $[\mathrm{M}+\mathrm{H}]^{+} 212.1075$, found 212.1078 .<smiles>CC(=CC(O)c1ccccc1)c1ccccc1</smiles>

(E)-1,3-diphenylbut-2-en-1-ol (11) 
Prepared according to the general procedure method $1 \mathrm{~h}$, yellow oil, $1.950 \mathrm{mg}, 61 \% .{ }^{\mathbf{1}} \mathbf{H} \mathbf{N M R}(400 \mathrm{MHz}$, $\left.\mathrm{CDCl}_{3}\right) \delta 7.46-7.24(\mathrm{~m}, 10 \mathrm{H}), 6.01(\mathrm{dq}, J=8.6,1.2 \mathrm{~Hz}, 1 \mathrm{H}), 5.65(\mathrm{~d}, J=8.6 \mathrm{~Hz}, 1 \mathrm{H}), 2.20(\mathrm{~d}, J=1.2$ $\mathrm{Hz}, 3 \mathrm{H}), 2.00(\mathrm{~s}, 1 \mathrm{H}){ }^{8}$

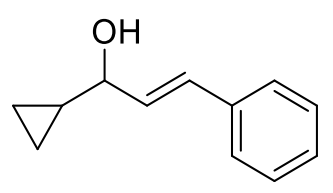

(E)-1-cyclopropyl-3-phenylprop-2-en-1-ol (1m)

Prepared according to the general procedure method $1 \mathrm{~h}$, colorless oil, $490 \mathrm{mg}, 100 \%$. ${ }^{1} \mathbf{H}$ NMR (400 $\left.\mathrm{MHz} \mathrm{CDCl}_{3}\right) \delta 7.41-7.39(\mathrm{~m}, 2 \mathrm{H}), 7.32(\mathrm{t}, J=7.6 \mathrm{~Hz}, 2 \mathrm{H}), 7.26-7.22(\mathrm{~m}, 1 \mathrm{H}), 5.61(\mathrm{~d}, J=16.0 \mathrm{~Hz}, 1 \mathrm{H})$, $6.32(\mathrm{dd}, J=16.0,6.2 \mathrm{~Hz}), 3.65(\mathrm{t}, J=7.2,1 \mathrm{H}), 1.82(\mathrm{~s}, 1 \mathrm{H}), 1.13-1.05(\mathrm{~m}, 1 \mathrm{H}), 0.64-0.54(\mathrm{~m}, 2 \mathrm{H})$, $0.45-0.40(\mathrm{~m}, 1 \mathrm{H}), 0.36-0.30(\mathrm{~m}, 1 \mathrm{H}){ }^{10}$<smiles>OC(/C=C/c1ccccc1)C1CCCCC1</smiles>

\section{(E)-1-cyclohexyl-3-phenylprop-2-en-1-ol (1n)}

Prepared according to the general procedure method $1 \mathrm{~h}$, yellow oil, 1.3576g, 63\%. ${ }^{1} \mathbf{H}$ NMR $(400 \mathrm{MHz}$, $\left.\mathrm{CDCl}_{3}\right) \delta 7.40-7.38(\mathrm{~m}, 2 \mathrm{H}), 7.33-7.29(\mathrm{~m}, 2 \mathrm{H}), 7.24-7.22(\mathrm{~m}, 1 \mathrm{H}), 6.55(\mathrm{~d}, J=16.0 \mathrm{~Hz}, 1 \mathrm{H}), 6.23(\mathrm{dd}$, $J=16.0,7.0 \mathrm{~Hz}, 1 \mathrm{H}), 4.02(\mathrm{t}, \mathrm{J}=6.8 \mathrm{~Hz}, 1 \mathrm{H}), 1.92(\mathrm{~d}, \mathrm{~J}=7.0 \mathrm{~Hz}, 1 \mathrm{H}), 1.80-1.65(\mathrm{~m}, 4 \mathrm{H}), 1.55-1.46(\mathrm{~m}$, $1 \mathrm{H}), 1.31-1.00(\mathrm{~m} 6 \mathrm{H}) .^{11}$<smiles>C=CC(O)c1cccc(Cl)c1</smiles>

\section{1-(3-chlorophenyl)prop-2-en-1-ol (10)}

Prepared according to the general procedure method $3 \mathrm{~h}$, yellow oil, $856 \mathrm{mg}, 98 \% .{ }^{1} \mathbf{H}$ NMR $(400 \mathrm{MHz}$, $\left.\mathrm{CDCl}_{3}\right) \delta 7.38-7.37(\mathrm{~m}, 1 \mathrm{H}), 7.31-7.23(\mathrm{~m}, 3 \mathrm{H}), 6.00(\mathrm{ddd}, J=16.8,10.4,6.4 \mathrm{~Hz}, 1 \mathrm{H}), 5.36(\mathrm{dt}, J=17.0$ $1.4 \mathrm{~Hz}, 1 \mathrm{H}), 5.23(\mathrm{dt}, J=10.4,1.2 \mathrm{~Hz}, 1 \mathrm{H}), 5.18(\mathrm{~s}, 1 \mathrm{H}), 2.10(\mathrm{br} \mathrm{s}, 1 \mathrm{H}){ }^{12}$<smiles>C=CC(O)c1ccc(C)cc1</smiles>

\section{1-(p-tolyl)prop-2-en-1-ol (1p)}

Prepared according to the general procedure method $3 \mathrm{~h}$, yellow oil, $616 \mathrm{mg}, 62 \% .{ }^{1} \mathbf{H}$ NMR $(400 \mathrm{MHz}$, $\left.\mathrm{CDCl}_{3}\right) \delta 7.26(\mathrm{~d}, J=8.0 \mathrm{~Hz}, 2 \mathrm{H}), 7.17(\mathrm{~d}, J=8.0 \mathrm{~Hz}, 2 \mathrm{H}), 6.05(\mathrm{ddd}, J=16.8,10.4,6.4 \mathrm{~Hz}, 1 \mathrm{H}), 5.34$ 
$(\mathrm{dt}, J=16.8,1.4 \mathrm{~Hz}, 1 \mathrm{H}), 5.20-5.17(\mathrm{~m}, 2 \mathrm{H}), 2.35(\mathrm{~s}, 3 \mathrm{H}), 1.93(\mathrm{~d} . J=3.6 \mathrm{~Hz}, 1 \mathrm{H}) .{ }^{13}$<smiles>C=CC(O)c1ccc(C(F)(F)F)cc1</smiles>

\section{1-(4-(trifluoromethyl)phenyl)prop-2-en-1-ol (1q)}

Prepared according to the general procedure method $3 \mathrm{~h}$, yellow oil, $696 \mathrm{mg}, 69 \% .{ }^{\mathbf{1}} \mathbf{H} \mathbf{~ N M R}(400 \mathrm{MHz}$, $\left.\mathrm{CDCl}_{3}\right) \delta 7.61(\mathrm{~d}, J=8.4 \mathrm{~Hz}, 2 \mathrm{H}), 7.50-7.48(\mathrm{~m}, 2 \mathrm{H}), 6.05-5.95(\mathrm{~m}, 1 \mathrm{H}), 5.39-5.33(\mathrm{~m}, 1 \mathrm{H}), 5.25-5.21$ $(\mathrm{m}, 1 \mathrm{H}), 2.40$ (br s, 1H). ${ }^{13}$<smiles>C=CC(O)c1ccc2ccccc2c1</smiles>

\section{1-(naphthalen-2-yl)prop-2-en-1-ol (1r)}

Prepared according to the general procedure method $1 \mathrm{~h}$, colorless oil, $1.026 \mathrm{~g}, 56 \% .{ }^{1} \mathbf{H ~ N M R}(400 \mathrm{MHz}$, $\left.\mathrm{CDCl}_{3}\right) \delta 7.87-7.82(\mathrm{~m}, 4 \mathrm{H}), 7.53-7.47(\mathrm{~m}, 3 \mathrm{H}), 6.13(\mathrm{ddd}, J=16.4,10.4,6.0 \mathrm{~Hz}, 1 \mathrm{H}), 5.41(\mathrm{dt}, J=17.2$, $1.4 \mathrm{~Hz}, 1 \mathrm{H}), 5.34(\mathrm{~s}, 1 \mathrm{H}), 5.25(\mathrm{dt}, J=10.4,1.4 \mathrm{~Hz}, 1 \mathrm{H}), 2.60(\mathrm{br} \mathrm{s}, 1 \mathrm{H}){ }^{13}$<smiles>C=CC(O)c1ccccn1</smiles>

\section{1-(pyridin-2-yl)prop-2-en-1-ol (1s)}

Prepared according to the general procedure method $1 \mathrm{~h}$, yellow oil, $427 \mathrm{mg}, 63 \% .{ }^{1} \mathbf{H} \mathbf{N M R}(400 \mathrm{MHz}$, $\left.\mathrm{CDCl}_{3}\right) \delta 8.55-8.53(\mathrm{~m}, 1 \mathrm{H}), 7.69(\mathrm{td}, J=7.6,1.8 \mathrm{~Hz}, 1 \mathrm{H}), 7.30(\mathrm{dd}, J=7.8,0.6 \mathrm{~Hz}, 1 \mathrm{H}), 7.23-7.19(\mathrm{~m}$, 1H), 5.97 (ddd, $J=17.0,10.2,6.8 \mathrm{~Hz}, 1 \mathrm{H}), 5.45$ (dt, $J=17.0,1.2 \mathrm{~Hz}, 1 \mathrm{H}), 5.24$ (dt, $J=10.0,1.2 \mathrm{~Hz}, 1 \mathrm{H})$, $5.18(\mathrm{~d}, J=6.0 \mathrm{~Hz}, 1 \mathrm{H}), 4.79(\mathrm{~s}, 1 \mathrm{H}) .{ }^{14}$<smiles>C=CC(O)c1ccc(Cl)cc1</smiles>

\section{1-(4-chlorophenyl)prop-2-en-1-ol (1t)}

Prepared according to the general procedure method $1 \mathrm{~h}$, yellow oil, $665 \mathrm{mg}, 66 \%$. ${ }^{1} \mathbf{H}$ NMR $(400 \mathrm{MHz}$, $\left.\mathrm{CDCl}_{3}\right) \delta$ 7.34-7.28 (m, 4H), 6.04-5.95 (m, 1H), $5.33(\mathrm{dq}, J=17.0,1.6 \mathrm{~Hz}, 1 \mathrm{H}), 5.22-5.16(\mathrm{~m}, 2 \mathrm{H}), 2.14$ (brs, $1 \mathrm{H}){ }^{15}$

\subsubsection{Preparation of starting materials 4}




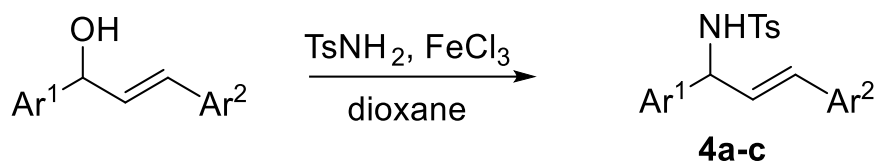

Compounds 4a-c were prepared as described in a reported protocol. ${ }^{4}$ General procedure: a corresponding allylic alcohol (1.0 equiv) was dissolved in dioxane in the open air, $\mathrm{TsNH}_{2}$ (2.0 equiv) and $\mathrm{FeCl}_{3}(0.05$ equiv) was added. The reaction mixture was then stirred for $24 \mathrm{~h}$ at room temperature. The reaction was quenched by adding saturated $\mathrm{NaCl}$ aq. and extracted with ethyl acetate. The combined organic extracts were dried over anhydrous $\mathrm{MgSO}_{4}$, filtered, concentrated and purified on silica gel chromatography (EtOAc/petroleum ether as eluent) to give the allylic amine.<smiles>[NH3+]C(/C=C/c1ccccc1)c1ccccc1</smiles>

\section{(E)-N-(1,3-diphenylallyl)-4-methylbenzenesulfonamide (4a)}

Prepared according to the general procedure method $24 \mathrm{~h}$, white solid, $808 \mathrm{mg}, 89 \% .{ }^{1} \mathbf{H} \mathbf{~ N M R}(400 \mathrm{MHz}$, $\left.\mathrm{CDCl}_{3}\right) \delta 7.67(\mathrm{~d}, J=8.4 \mathrm{~Hz}, 2 \mathrm{H}), 7.30-7.16(\mathrm{~m}, 12 \mathrm{H}), 6.37(\mathrm{~d}, J=15.6 \mathrm{~Hz}, 1 \mathrm{H}), 6.09(\mathrm{dd}, J=15.6,6.8$ $\mathrm{Hz}, 1 \mathrm{H}), 5.13(\mathrm{t}, J=6.6 \mathrm{~Hz}, 1 \mathrm{H}), 4.82(\mathrm{~d}, J=7.2 \mathrm{~Hz}, 1 \mathrm{H}), 2.35(\mathrm{~s}, 3 \mathrm{H}) .{ }^{16}$<smiles>Cc1ccc(/C=C/C([NH3+])c2ccc(C)cc2)cc1</smiles>

\section{(E)-N-(1,3-di-p-tolylallyl)-4-methylbenzenesulfonamide (4b)}

Prepared according to the general procedure method $24 \mathrm{~h}$, white solid, $618 \mathrm{mg}, 63 \%$. ${ }^{1} \mathbf{H}$ NMR $(400 \mathrm{MHz}$, $\left.\mathrm{CDCl}_{3}\right) \delta 7.65(\mathrm{~d}, J=8.4 \mathrm{~Hz}, 2 \mathrm{H}), 7.15(\mathrm{~d}, J=8.0 \mathrm{~Hz}, 2 \mathrm{H}), 7.09-7.04(\mathrm{~m}, 8 \mathrm{H}), 6.31(\mathrm{dd}, J=15.6,1.0$ $\mathrm{Hz}, 1 \mathrm{H}), 6.01(\mathrm{dd}, J=16.0,6.8 \mathrm{~Hz}, 1 \mathrm{H}), 5.06(\mathrm{t}, J=6.8 \mathrm{~Hz}, 1 \mathrm{H}), 4.86(\mathrm{~d}, J=6.8 \mathrm{~Hz}, 1 \mathrm{H}), 2.34(\mathrm{~s}, 3 \mathrm{H})$, $2.32(\mathrm{~s}, 3 \mathrm{H}), 2.30(\mathrm{~s}, 3 \mathrm{H}) .^{17}$<smiles>[NH3+]C(/C=C/c1cccc(Cl)c1)c1ccccc1</smiles>

\section{(E)-N-(3-(3-chlorophenyl)-1-phenylallyl)-4-methylbenzenesulfonamide (4c)}

Prepared according to the general procedure method $24 \mathrm{~h}$, white solid, $556 \mathrm{mg}, 67 \%$. ${ }^{1} \mathbf{H}$ NMR $(400 \mathrm{MHz}$, $\left.\mathrm{CDCl}_{3}\right) \delta 7.68(\mathrm{~d}, J=8.0 \mathrm{~Hz}, 2 \mathrm{H}), 7.29-7.06(\mathrm{~m}, 12 \mathrm{H}), 6.32(\mathrm{~d}, J=7.6 \mathrm{~Hz}, 1 \mathrm{H}), 6.08(\mathrm{dd}, J=16.0,6.8$ $\mathrm{Hz}, 1 \mathrm{H}), 5.13(\mathrm{t}, J=6.8 \mathrm{~Hz}, 1 \mathrm{H}), 4.92(\mathrm{~d}, J=6.8 \mathrm{~Hz}, 1 \mathrm{H}), 2.37$ (s, 3H). ${ }^{18}$

\subsubsection{General procedure for allylic isomerization of alcohols}


Allylic alcohol $1(0.5 \mathrm{mmol})$ was weighed directly into a Schlenk tube. After drying under high vacuum for $15 \mathrm{~min}$, toluene $(1 \mathrm{~mL})$ was added, followed by the addition of ${ }^{t} \mathrm{BuONa}$ and 1,10-phenanthroline under stirring. The resulting reaction mixture was stirred at $80{ }^{\circ} \mathrm{C}$ and monitored by TLC. The crude reaction mixture was directly purified by silica gel column (EtOAc/petroleum ether as eluent).

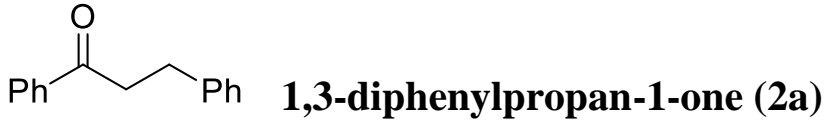

Prepared according to the general procedure method $1 \mathrm{~h}$, white solid, $101 \mathrm{mg}, 96 \% .{ }^{1} \mathbf{H}$ NMR $(400 \mathrm{MHz}$, $\left.\mathrm{CDCl}_{3}\right) \delta$ 7.98-7.95 (m, 2H), 7.58-7.54 (m, 1H), 7.48-7.44 (m, 2H), 7.33-7.19(m, 5H), 3.33-3.30 (m, $2 \mathrm{H}), 3.10-3.06(\mathrm{~m}, 2 \mathrm{H}){ }^{19}$

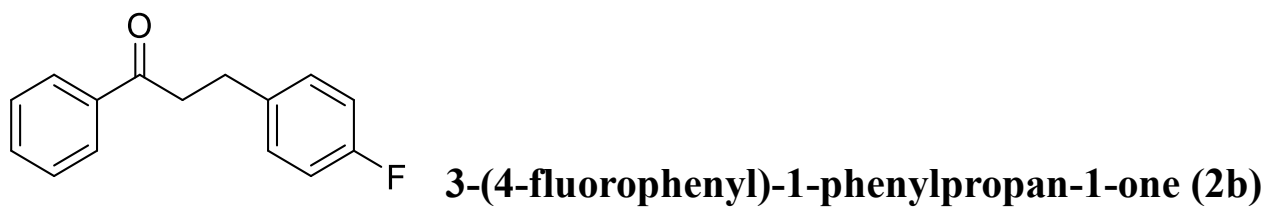

Prepared according to the general procedure method $3 \mathrm{~h}$, white solid, $98.2 \mathrm{mg}, 86 \% .{ }^{1} \mathbf{H}$ NMR $(400 \mathrm{MHz}$, $\left.\mathrm{CDCl}_{3}\right) \delta$ 7.97-7.94 (m, 2H), $7.56(\mathrm{tt}, J=7.4,1.4 \mathrm{~Hz}, 1 \mathrm{H}), 7.48-7.43(\mathrm{~m}, 2 \mathrm{H}), 7.23-7.19(\mathrm{~m}, 2 \mathrm{H}), 7.00-$ $6.95(\mathrm{~m}, 2 \mathrm{H}), 3.30-3.27(\mathrm{~m}, 2 \mathrm{H}), 3.05(\mathrm{t}, J=7.6 \mathrm{~Hz}, 2 \mathrm{H}){ }^{\mathbf{2 0}}$<smiles>C/C=C/CCC(=O)c1ccccc1</smiles>

\section{(E)-1-phenylhex-4-en-1-one (2c)}

Prepared according to the general procedure method using $20 \mathrm{~mol} \%$ of $\mathbf{3 a}-{ }^{t} \mathrm{BuONa}$ for $4 \mathrm{~h}$, yellow oil, $72.4 \mathrm{mg}, 83 \% .{ }^{1} \mathbf{H}$ NMR $\left(400 \mathrm{MHz}, \mathrm{CDCl}_{3}\right) \delta$ 7.97-7.95 (m, 2H), $7.55(\mathrm{t}, J=7.4 \mathrm{~Hz}, 1 \mathrm{H}), 7.46(\mathrm{t}, J=$ $7.8 \mathrm{~Hz}, 2 \mathrm{H}), 7.52-7.49(\mathrm{~m}, 2 \mathrm{H}), 3.03(\mathrm{t}, J=7.4 \mathrm{~Hz}, 2 \mathrm{H}), 2.45-2.39(\mathrm{~m}, 1 \mathrm{H}), 1.65-1.64(\mathrm{~m}, 3 \mathrm{H}){ }^{21}$<smiles>O=C(CCc1ccccc1)c1ccc(Cl)cc1</smiles>

\section{1-(4-chlorophenyl)-3-phenylpropan-1-one (2d)}

Prepared according to the general procedure method $2 \mathrm{~h}$, white solid, $105 \mathrm{mg}, 86 \% .{ }^{1} \mathbf{H}$ NMR $(400 \mathrm{MHz}$, $\left.\mathrm{CDCl}_{3}\right) \delta 7.89(\mathrm{dt}, J=9.2,2.2 \mathrm{~Hz}, 2 \mathrm{H}), 7.43(\mathrm{dt}, J=9.2,2.2 \mathrm{~Hz}, 2 \mathrm{H}), 7.33-7.28(\mathrm{~m}, 2 \mathrm{H}), 7.26-7.19$ (m, $3 \mathrm{H}) 3.29-3.25(\mathrm{~m}, 2 \mathrm{H}), 3.06(\mathrm{t}, J=7.8 \mathrm{~Hz}, 2 \mathrm{H}){ }^{20}$<smiles>O=C(CCc1ccccc1)c1cccc(Cl)c1</smiles> 
Prepared according to the general procedure method $1 \mathrm{~h}$, yellow oil, xxx mg, 93\%. ${ }^{1} \mathbf{H}$ NMR $(400 \mathrm{MHz}$, $\left.\mathrm{CDCl}_{3}\right) \delta 7.91(\mathrm{~s}, 1 \mathrm{H}), 7.81(\mathrm{~d}, J=7.6 \mathrm{~Hz}, 1 \mathrm{H}), 7.51(\mathrm{ddd}, J=8.0,2.2,1.0 \mathrm{~Hz}, 1 \mathrm{H}), 7.37(\mathrm{t}, J=8.0 \mathrm{~Hz}$ $1 \mathrm{H}), 7.31-7.18(\mathrm{~m}, 5 \mathrm{H}), 3.28-3.24(\mathrm{~m}, 2 \mathrm{H}), 3.05(\mathrm{t}, J=7.6 \mathrm{~Hz}, 2 \mathrm{H}){ }^{22}$<smiles>COc1ccc(C(=O)CCc2ccccc2)cc1</smiles>

\section{1-(4-methoxyphenyl)-3-phenylpropan-1-one (2f)}

Prepared according to the general procedure method using $20 \mathrm{~mol} \%$ of $\mathbf{3 a}{ }^{t}{ }^{t} \mathrm{BuONa}$ for $4 \mathrm{~h}$, white solid, $92.6 \mathrm{mg}, 77 \% .{ }^{1} \mathbf{H}$ NMR (400 MHz, CDCl3) $\delta 7.95$ (dt, J=9.6, $\left.2.4 \mathrm{~Hz}, 2 \mathrm{H}\right), 7.32-7.18$ (m, 5H), 6.93 (dt, $J=9.6,2.4 \mathrm{~Hz}, 2 \mathrm{H}), 3.87(\mathrm{~s}, 3 \mathrm{H}), 3.27-3.23(\mathrm{~m}, 2 \mathrm{H}), 3.08-3.04(\mathrm{~m}, 2 \mathrm{H}){ }^{20}$<smiles>Cc1ccc(C(=O)CCc2ccccc2)cc1</smiles>

\section{3-phenyl-1-(p-tolyl)propan-1-one (2g)}

Prepared according to the general procedure method using $20 \mathrm{~mol} \%$ of $\mathbf{3 a}-{ }^{t} \mathrm{BuONa}$ for $1 \mathrm{~h}$, white solid, $88 \mathrm{mg}, 78 \%{ }^{1} \mathbf{H}$ NMR (400 MHz, $\left.\mathrm{CDCl}_{3}\right) \delta 7.86(\mathrm{dd}, J=8.2,1.8 \mathrm{~Hz}, 2 \mathrm{H}), 7.32-7.18$ (m, 7H), 3.29-3.25 $(\mathrm{m}, 2 \mathrm{H}), 3.06(\mathrm{t}, J=7.6 \mathrm{~Hz}, 2 \mathrm{H}), 2.40(\mathrm{~s}, 3 \mathrm{H}){ }^{20}$

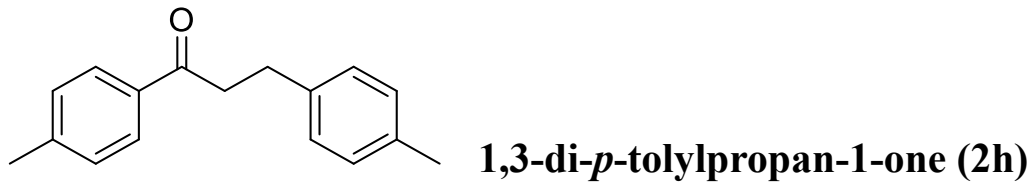

Prepared according to the general procedure method using $20 \mathrm{~mol} \%$ of $\mathbf{3 a}-{ }^{t} \mathrm{BuONa}$ for $1 \mathrm{~h}$, white solid, $93.1 \mathrm{mg}, 78 \% .{ }^{1} \mathbf{H}$ NMR (400 MHz, $\left.\mathrm{CDCl}_{3}\right) \delta$ 7.87-7.84 (m, 2H), 7.25-7.23 (m, 2H), 7.16-7.09 (m, 4H), $3.27-3.22(\mathrm{~m}, 2 \mathrm{H}), 3.02(\mathrm{t}, J=7.6 \mathrm{~Hz}, 2 \mathrm{H}), 2.40(\mathrm{~s}, 3 \mathrm{H}), 2.32(\mathrm{~s}, 3 \mathrm{H}) .^{23}$

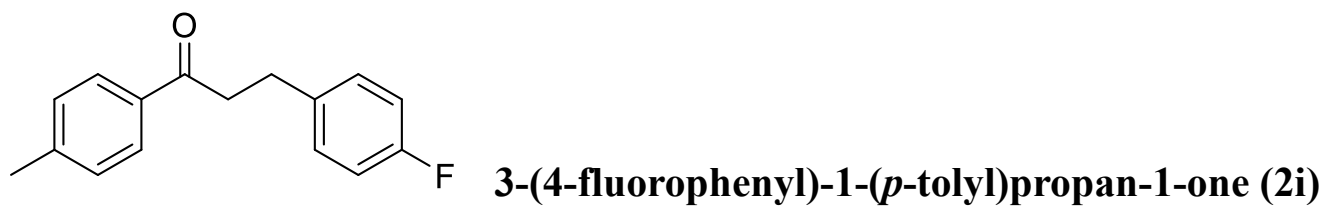

Prepared according to the general procedure method $2 \mathrm{~h}$, white solid, $97.3 \mathrm{mg}, 81 \% .{ }^{1} \mathbf{H} \mathbf{N M R}(400 \mathrm{MHz}$, $\left.\mathrm{CDCl}_{3}\right) \delta 7.85(\mathrm{dt}, J=8.4,1.8 \mathrm{~Hz}, 2 \mathrm{H}), 7.26-7.19(\mathrm{~m}, 4 \mathrm{H}), 7.00-6.94(\mathrm{~m}, 2 \mathrm{H}), 3.27-3.23(\mathrm{~m}, 2 \mathrm{H}), 3.04$ (t, $J=7.6 \mathrm{~Hz}, 2 \mathrm{H}), 2.41(\mathrm{~s}, 3 \mathrm{H}) .{ }^{13} \mathbf{C} \mathbf{N M R}\left(100 \mathrm{MHz}, \mathrm{CDCl}_{3}\right) \delta 198.8,162.6,160.4,144.0,137.1(\mathrm{~d}, J=$ $15.2 \mathrm{~Hz}), 134.4,129.9(\mathrm{~d}), 129.4,128.2,115.4,115.2,40.4,29.4,21.7$. HRMS (ESI) calcd for $\mathrm{C}_{16} \mathrm{H}_{16} \mathrm{FO}^{+}$ $[\mathrm{M}+\mathrm{H}]^{+}$243.1180, found 243.1179. 
<smiles>Cc1ccc(C(=O)CCc2ccc(C(F)(F)F)cc2)cc1</smiles>

\section{1-(p-tolyl)-3-(4-(trifluoromethyl)phenyl)propan-1-one (2j)}

Prepared according to the general procedure method, 2 h, white solid, $139 \mathrm{mg}, 95 \%{ }^{1} \mathbf{H} \mathbf{~ N M R}(400 \mathrm{MHz}$, $\left.\mathrm{CDCl}_{3}\right) \delta 7.86(\mathrm{dt}, J=8.6,1.8 \mathrm{~Hz}, 2 \mathrm{H}), 7.55(\mathrm{~d}, J=8.0 \mathrm{~Hz}, 2 \mathrm{H}), 7.37(\mathrm{~d}, J=8.0 \mathrm{~Hz}, 2 \mathrm{H}), 7.26(\mathrm{~d}, J=8.0$ $\mathrm{Hz}, 2 \mathrm{H}), 3.32-3.28(\mathrm{~m}, 2 \mathrm{H}), 3.13(\mathrm{t}, J=7.4 \mathrm{~Hz}, 2 \mathrm{H}) .{ }^{13} \mathbf{C ~ N M R}\left(100 \mathrm{MHz}, \mathrm{CDCl}_{3}\right) \delta 198.3,145.6,144.2$, 134.1, 129.4, 128.6, 128.1, 125.4, 39.7, 29.6, 21.7. HRMS (ESI) calcd for $\mathrm{C}_{17} \mathrm{H}_{16} \mathrm{~F}_{3} \mathrm{O}^{+}[\mathrm{M}+\mathrm{H}]^{+}$293.1148, found 293.1139 .<smiles>O=C(CCc1ccccn1)c1ccccc1</smiles>

\section{1-phenyl-3-(pyridin-2-yl)propan-1-one (2k)}

Prepared according to the general procedure method using $20 \mathrm{~mol} \%$ of $\mathbf{3 a}-{ }^{t} \mathrm{BuONa}$ for $\mathrm{h} 3 \mathrm{~h}$, yellow oil, $91 \mathrm{mg}, 86 \%{ }^{1}{ }^{1} \mathrm{H}$ NMR $\left(400 \mathrm{MHz}, \mathrm{CDCl}_{3}\right) \delta 8.51(\mathrm{~d}, J=4.8 \mathrm{~Hz}, 2 \mathrm{H}), 8.00(\mathrm{~d}, J=8.0 \mathrm{~Hz}, 2 \mathrm{H}), 7.60-7.52$ $(\mathrm{m}, 2 \mathrm{H}), 7.44(\mathrm{t}, J=7.6 \mathrm{~Hz}, 2 \mathrm{H}), 7.26(\mathrm{~d}, J=8.0 \mathrm{~Hz}, 1 \mathrm{H}), 7.10(\mathrm{t}, J=6.2 \mathrm{~Hz}, 1 \mathrm{H}), 3.51(\mathrm{t}, J=7.2 \mathrm{~Hz}$ $2 \mathrm{H}), 3.24(\mathrm{t}, J=7.2 \mathrm{~Hz}, 2 \mathrm{H}){ }^{24}$<smiles>CC(CC(=O)c1ccccc1)c1ccccc1</smiles>

\section{1-phenyl-3-phenylbutan-1-one (2l)}

Prepared according to the general procedure method using $20 \mathrm{~mol} \%$ of $\mathbf{3 a}{ }^{t}{ }^{t} \mathrm{BuONa}$ at $110^{\circ} \mathrm{C}, 6 \mathrm{~h}$, yellow solid, $72.2 \mathrm{mg}, 64 \% .{ }^{1} \mathbf{H}$ NMR (400 $\mathrm{MHz}^{\left.\mathrm{CDCl}_{3}\right)} \delta 7.95-7.92(\mathrm{~m}, 2 \mathrm{H}), 7.55(\mathrm{tt}, J=7.4,1.4 \mathrm{~Hz}, 1 \mathrm{H})$, 7.46-7.42 (m, 2H), 7.33-7.22 (m, 4H), 7.21-7.18 (m, 1H), 3.56-3.47 (m, 1H), 3.31 (dd, J=16.4, 3.8 Hz, $1 \mathrm{H}), 3.19(\mathrm{dd}, J=16.4,8.4 \mathrm{~Hz}, 1 \mathrm{H}), 1.35(\mathrm{~d}, J=6.8 \mathrm{~Hz}, 3 \mathrm{H}){ }^{25}$<smiles>O=C(CCc1ccccc1)C1CC1</smiles>

\section{1-cyclopropyl-3-phenylpropan-1-one (2m)}

Prepared according to the general procedure method using $20 \mathrm{~mol} \%$ of $\mathbf{3 a}$ and $50 \mathrm{~mol} \%$ of ${ }^{t} \mathrm{BuONa}$ at $110^{\circ} \mathrm{C}, 3 \mathrm{~h}$, colorless oil, $63.3 \mathrm{mg}, 73 \% .{ }^{1} \mathbf{H}$ NMR (400 MHz, $\left.\mathrm{CDCl}_{3}\right) \delta$ 7.30-7.26 (m, 2H), 7.20-7.17 $(\mathrm{m}, 3 \mathrm{H}), 2.95-9.85(\mathrm{~m}, 4 \mathrm{H}), 1.94-1.88(\mathrm{~m}, 1 \mathrm{H}), 1.04-1.00(\mathrm{~m}, 1 \mathrm{H}), 0.88-0.830(\mathrm{~m}, 1 \mathrm{H}){ }^{26}$ 
<smiles>O=C(CCc1ccccc1)C1CCCCC1</smiles>

\section{1-cyclopropyl-3-phenylpropan-1-one (2n)}

Prepared according to the general procedure method using $20 \mathrm{~mol} \%$ of 3a and $100 \mathrm{~mol} \%$ of ${ }^{t} \mathrm{BuONa}, 4$ h, colorless oil, $52.8 \mathrm{mg}, 73 \%$. ${ }^{1} \mathbf{H}$ NMR (400 MHz, $\left.\mathrm{CDCl}_{3}\right) \delta 7.29-7.25$ (m, 2H), 7.19-7.16 (m, 3H), 2.88 $(\mathrm{t}, \mathrm{J}=7.4 \mathrm{~Hz}, 2 \mathrm{H}), 2.77-2.73(\mathrm{~m}, 2 \mathrm{H}), 2.34-2.27$ (m, 1H), 1.81-1.74 (m, 4H), 1.66-1.63 (m, 1H), 1.35$1.21(\mathrm{~m}, 5 \mathrm{H}){ }^{27}$<smiles>CCC(=O)c1cccc(Cl)c1</smiles>

\section{1-(3-chlorophenyl)propan-1-one (20)}

Prepared according to the general procedure method $3 \mathrm{~h}$, colorless oil, $66.8 \mathrm{mg}, 80 \% .{ }^{1} \mathbf{H ~ N M R}(400 \mathrm{MHz}$, $\left.\mathrm{CDCl}_{3}\right) \delta 7.93(\mathrm{t}, J=1.8 \mathrm{~Hz}, 1 \mathrm{H}), 7.83(\mathrm{dt}, J=8.0,1.2 \mathrm{~Hz}, 1 \mathrm{H}), 7.52(\mathrm{dq}, J=8.0,1.0 \mathrm{~Hz}, 1 \mathrm{H}), 7.40(\mathrm{t}, J$ $=8.0 \mathrm{~Hz}, 1 \mathrm{H}), 2.98(\mathrm{q}, J=7.2 \mathrm{~Hz}, 2 \mathrm{H}), 1.23(\mathrm{t}, J=7.2 \mathrm{~Hz}, 3 \mathrm{H}){ }^{28}$<smiles>CCC(=O)c1ccc(C)cc1</smiles>

\section{1-(p-tolyl)propan-1-one (2p)}

Prepared according to the general procedure method $4 \mathrm{~h}$, colorless oil, $55 \mathrm{mg}, 74 \% .{ }^{1} \mathbf{H} \mathbf{~ N M R}(400 \mathrm{MHz}$, $\left.\mathrm{CDCl}_{3}\right) \delta 7.87(\mathrm{~d}, J=8.0 \mathrm{~Hz}, 2 \mathrm{H}), 7.26(\mathrm{~d}, J=8.0 \mathrm{~Hz}, 3 \mathrm{H}), 2.99(\mathrm{q}, J=7.2 \mathrm{~Hz}, 2 \mathrm{H}), 2.42(\mathrm{~s}, 3 \mathrm{H}), 1.22(\mathrm{t}$, $J=7.2 \mathrm{~Hz}, 3 \mathrm{H}){ }^{29}$<smiles>CCC(=O)c1ccc(C(F)(F)F)cc1</smiles>

\section{1-(4-(trifluoromethyl)phenyl)propan-1-one (2q)}

Prepared according to the general procedure method $2 \mathrm{~h}$, colorless oil, $71 \mathrm{mg}, 88 \%{ }^{1} \mathbf{H} \mathbf{~ N M R}(400 \mathrm{MHz}$, $\left.\mathrm{CDCl}_{3}\right) \delta 8.07(\mathrm{~d}, J=8.0 \mathrm{~Hz}, 2 \mathrm{H}), 7.73(\mathrm{~d}, J=8.0 \mathrm{~Hz}, 2 \mathrm{H}), 3.04(\mathrm{q}, J=7.2 \mathrm{~Hz}, 2 \mathrm{H}), 1.25(\mathrm{t}, J=7.2 \mathrm{~Hz}$, $3 \mathrm{H}){ }^{30}$<smiles>CCC(=O)c1ccc2ccccc2c1</smiles>

\section{1-(naphthalen-2-yl)propan-1-one (2r)}

Prepared according to the general procedure method $2 \mathrm{~h}$, yellow solid, $73.6 \mathrm{mg}, 80 \% .{ }^{1} \mathbf{H ~ N M R}(400 \mathrm{MHz}$, $\left.\mathrm{CDCl}_{3}\right) \delta 8.46(\mathrm{~s}, 1 \mathrm{H}), 8.03(\mathrm{dd}, J=8.6,1.8 \mathrm{~Hz}, 1 \mathrm{H}), 7.95(\mathrm{~d}, J=8.0 \mathrm{~Hz}, 1 \mathrm{H}), 7.87(\mathrm{t}, J=8.0 \mathrm{~Hz}, 2 \mathrm{H})$, 


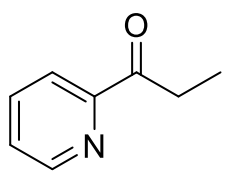

\section{1-(pyridin-2-yl)propan-1-one (2s)}

Prepared according to the general procedure method $1 \mathrm{~h}$, yellow oil, $57 \mathrm{mg}, 84 \% .{ }^{1} \mathbf{H}$ NMR $(400 \mathrm{MHz}$, $\left.\mathrm{CDCl}_{3}\right) \delta 8.69(\mathrm{dq}, J=4.8,0.8 \mathrm{~Hz}, 1 \mathrm{H}), 8.06(\mathrm{dt}, J=7.6,1.2 \mathrm{~Hz}, 1 \mathrm{H}), 7.84(\mathrm{td}, J=7.6,1.8 \mathrm{~Hz}, 1 \mathrm{H}), 7.47$ $(\mathrm{ddd}, J=7.6,4.8,1.6 \mathrm{~Hz}, 1 \mathrm{H}), 3.26(\mathrm{q}, J=7.2 \mathrm{~Hz}, 2 \mathrm{H}), 1.23(\mathrm{t}, J=7.2 \mathrm{~Hz}, 3 \mathrm{H}){ }^{32}$<smiles>CCC(=O)c1ccc(Cl)cc1</smiles>

\section{1-(4-chlorophenyl)propan-1-one (2t)}

Prepared according to the general procedure method $3 \mathrm{~h}$, yellow oil, $59.5 \mathrm{mg}, 71 \%{ }^{1} \mathbf{H} \mathbf{~ N M R}(400 \mathrm{MHz}$, $\left.\mathrm{CDCl}_{3}\right) \delta 7.90(\mathrm{dt}, J=8.8,2.4 \mathrm{~Hz}, 2 \mathrm{H}), 7.43(\mathrm{dt}, J=8.8,2.4 \mathrm{~Hz}, 2 \mathrm{H}), 2.97(\mathrm{q}, J=7.2 \mathrm{~Hz}, 2 \mathrm{H}), 1.22(\mathrm{t}, J$ $=7.2 \mathrm{~Hz}, 3 \mathrm{H}) .^{31}$

\subsubsection{General procedure for allylic isomerization of imines}

Allylic imine $4(0.25 \mathrm{mmol})$ was weighed directly into a Schlenk tube. After drying under high vacuum for $15 \mathrm{~min}$, toluene $(1 \mathrm{~mL})$ was added, followed by the addition of ${ }^{t} \mathrm{BuONa}$ and 1,10 -phenanthroline under stirring. The resulting reaction mixture was stirred at $110^{\circ} \mathrm{C}$ for $24 \mathrm{~h}$. The crude reaction mixture was directly purified by silica gel column (EtOAc/petroleum ether as eluent).

\section{$\overbrace{\mathrm{Ph}}^{\mathrm{O}} \overbrace{1,3-\text { diphenylpropan-1-one (2a) }}$}

Prepared according to the general procedure from 4a, ${ }^{t} \mathrm{BuONa}(0.125 \mathrm{mmol}, 50 \mathrm{~mol} \%), 1,10$-phenanthroline (0.05 mmol, $20 \mathrm{~mol} \%$ ), white solid, $46 \mathrm{mg}, 87 \%$. ${ }^{1} \mathbf{H} \mathbf{N M R}\left(400 \mathrm{MHz}, \mathrm{CDCl}_{3}\right) \delta 7.98-7.95(\mathrm{~m}$, 2H), 7.58-7.54 (m, 1H), 7.48-7.44 (m, 2H), 7.33-7.19(m, 5H), 3.33-3.30 (m, 2H), 3.10-3.06 (m, 2H). ${ }^{19}$<smiles>Cc1ccc(CCC(=O)c2ccc(C)cc2)cc1</smiles>

\section{1,3-di-p-tolylpropan-1-one (2h)}

Prepared according to the general procedure from $\mathbf{4 b},{ }^{t} \mathrm{BuONa}(0.25 \mathrm{mmol}), 1,10$-phenanthroline $(0.25$ mmol), white solid, $56 \mathrm{mg}, 93 \% .{ }^{1} \mathbf{H}$ NMR (400 MHz, $\left.\mathrm{CDCl}_{3}\right) \delta$ 7.87-7.84 (m, 2H), 7.25-7.23 (m, 2H), 7.16-7.09 (m, 4H), 3.27-3.22 (m, 2H), $3.02(\mathrm{t}, J=7.6 \mathrm{~Hz}, 2 \mathrm{H}), 2.40(\mathrm{~s}, 3 \mathrm{H}), 2.32(\mathrm{~s}, 3 \mathrm{H}) .{ }^{23}$ 
<smiles>O=C(CCc1cccc(Cl)c1)c1ccccc1</smiles>

\section{3-(3-chlorophenyl)-1-phenylpropan-1-one (2u)}

Prepared according to the general procedure method from $4 \mathbf{c},{ }^{t} \mathrm{BuONa}(0.25 \mathrm{mmol}), 1,10$-phenanthroline (0.125 mmol, $50 \mathrm{~mol} \%$ ), yellow solid, $50 \mathrm{mg}, 82 \% .{ }^{1} \mathbf{H}$ NMR (400 MHz, $\left.\mathrm{CDCl}_{3}\right) \delta 7.98-7.95(\mathrm{~m}, 2 \mathrm{H})$, 7.57 (tt, $J=7.2,1.4 \mathrm{~Hz}, 1 \mathrm{H}), 7.49-7.44(\mathrm{~m}, 2 \mathrm{H}), 7.26-7.13(\mathrm{~m}, 4 \mathrm{H}), 3.32-3.28(\mathrm{~m}, 2 \mathrm{H}), 3.05(\mathrm{t}, J=7.6$ $\mathrm{Hz}, 2 \mathrm{H}) .{ }^{33}$

\subsection{Synthesis of $5 a$ from $2 a($ from 1a)}

Ketone $2 \mathrm{a}(0.5 \mathrm{mmol})$ was added to a solution of $\mathrm{TfOH}(4.4 \mathrm{~mL})$ and heated to $80{ }^{\circ} \mathrm{C}$ for $22 \mathrm{~h}$. The reaction mixture was poured into a large excess of ice water and extracted with $\mathrm{Et}_{2} \mathrm{O}$. The organic layer was washed with brine, dried over $\mathrm{Na}_{2} \mathrm{SO}_{4}$, and concentrated under reduced pressure. The residue was purified by flash column chromatography (EtOAc/petroleum ether as eluent). yellow oil, $80.3 \mathrm{mg}, 83 \% .{ }^{34}$<smiles>C1=C(c2ccccc2)c2ccccc2C1</smiles>

\section{3-phenyl-1H-indene (5a)}

${ }^{1} \mathbf{H}$ NMR $\left(400 \mathrm{MHz}, \mathrm{CDCl}_{3}\right) \delta$ 7.60-7.58 (m, 3H), $7.52(\mathrm{~d}, J=7.2 \mathrm{~Hz}, 1 \mathrm{H}), 7.45-7.41$ (m, 2H), 7.37$7.29(\mathrm{~m}, 2 \mathrm{H}), 7.24(\mathrm{t}, J=3.2 \mathrm{~Hz}, 1 \mathrm{H}), 6.56(\mathrm{t}, J=2.0 \mathrm{~Hz}, 1 \mathrm{H}), 3.48(\mathrm{~s}, 2 \mathrm{H}){ }^{34}$ 
2.9. Monitoring deprotonation of $1 \mathrm{a}$ in large excess of ${ }^{t} \mathrm{BuONa}$

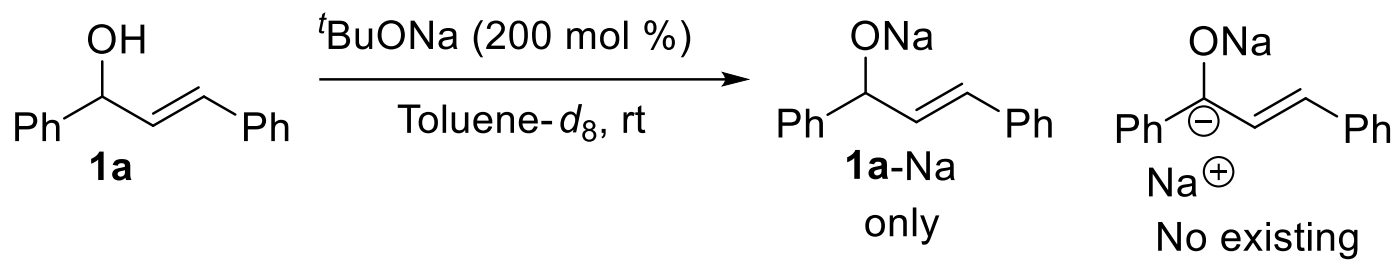

$1 \mathrm{a}(0.125 \mathrm{mmol})$ was weighed directly into a NMR tube and solvent (toluene- $\left.d_{8} 0.4 \mathrm{~mL}\right)$, was added and stirred. Then the reaction mixture was directly examined on ${ }^{1} \mathrm{H}$ NMR spectrometer. Then ${ }^{t} \mathrm{BuONa}$ (200 mol \%, $25 \mathrm{mg}$ ) was added and shaken vigorously. The reaction mixture was directly examined on ${ }^{1} \mathrm{H}$ NMR spectrometer and 1a-Na was observed as only product. There is no di-anion.
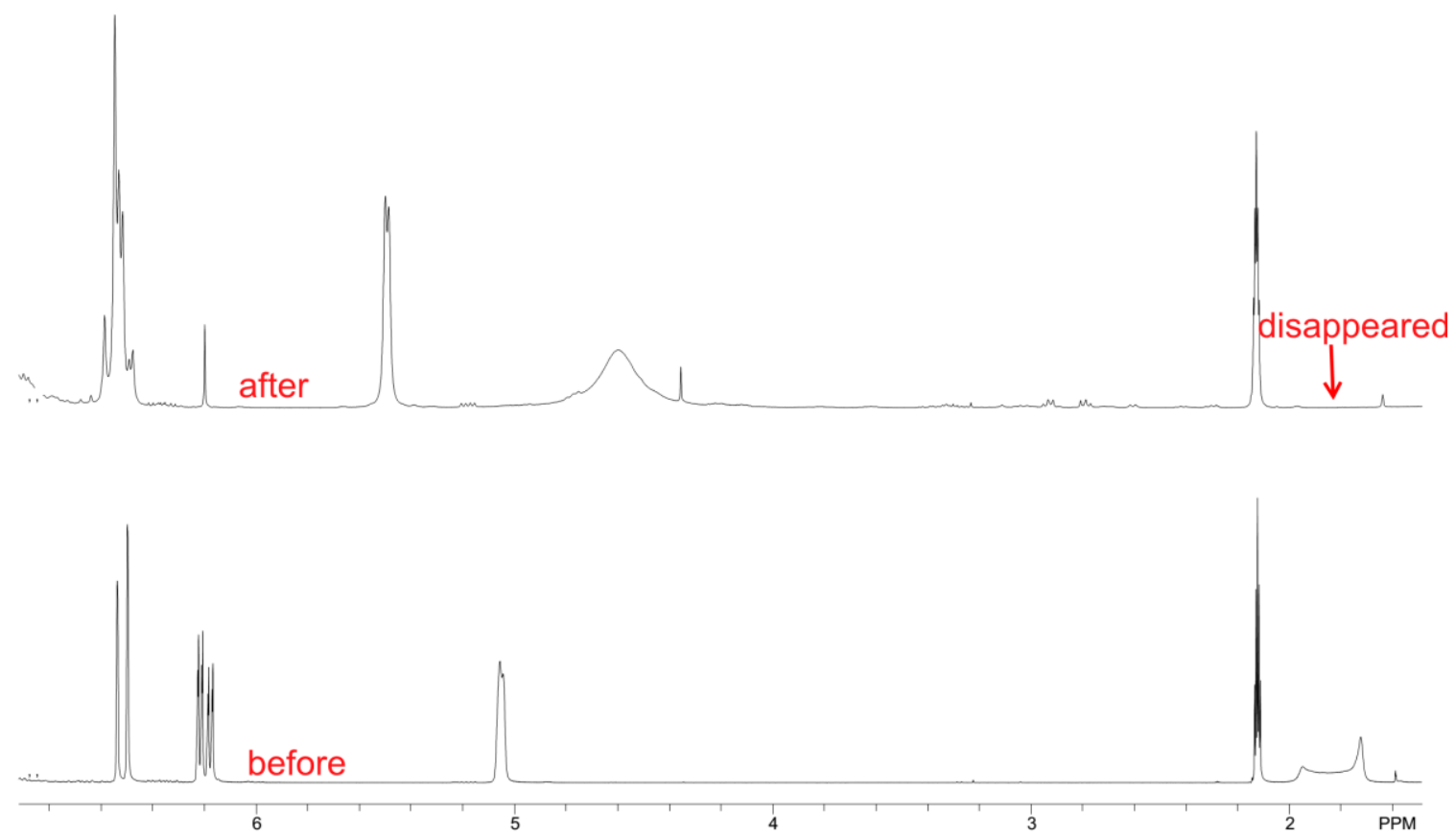

\section{Isotopic Labelling Experiments and Kinetic Studies}

\subsection{Isotopic Labelling Experiments}

Preparation of deuterated substrate 1a- $d$ :

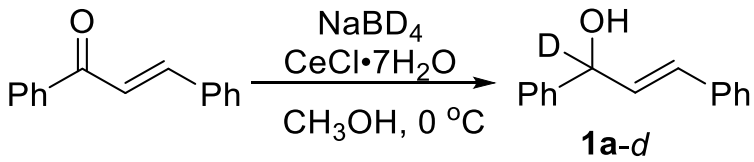

Chalcone was reduced with $\mathrm{NaBD}_{4}$ in the presence of $\mathrm{CeCl}_{3} \cdot 7 \mathrm{H}_{2} \mathrm{O}$. The reduction was quenched by addition of a mixed solution of saturated $\mathrm{NH}_{4} \mathrm{Cl}$ and $1 \mathrm{M} \mathrm{HCl}$ and extracted with $\mathrm{CH}_{2} \mathrm{Cl}_{2}$. The combined organic extracts were washed with brine, dried over anhydrous $\mathrm{MgSO}_{4}$, filtered, concentrated under reduced pressure and purified by silica gel chromatography to give the $1 \mathbf{a}-d .{ }^{1} \mathrm{H} \mathrm{NMR}\left(400 \mathrm{MHz}, \mathrm{CDCl}_{3}\right)$ 
$\delta$ 7.44-7.21 (m, 10H), $6.68(\mathrm{~d}, J=15.6 \mathrm{~Hz}, 1 \mathrm{H}), 6.38(\mathrm{~d}, J=16.0 \mathrm{~Hz}, 1 \mathrm{H}), 5.38(\mathrm{~d}, J=6.8 \mathrm{~Hz}, 1 \mathrm{H}), 2.12$ $(\mathrm{s}, 1 \mathrm{H})$.

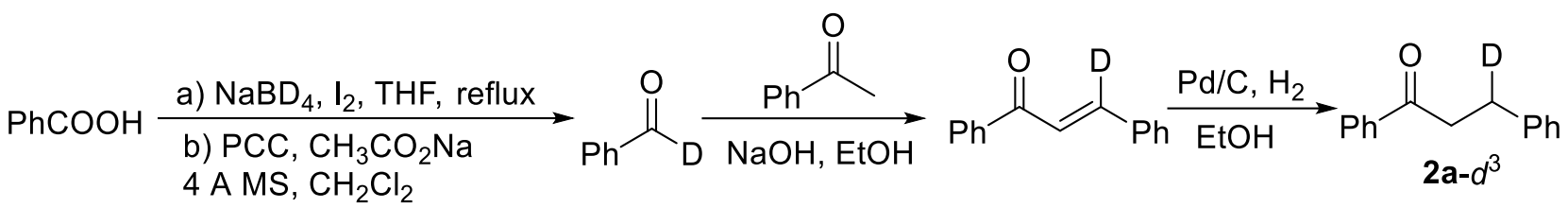

A $100 \mathrm{~mL}$ round-bottom flask was charged with benzoic acid (977 mg, $8 \mathrm{mmol})$ and THF (20 mL, predried over sodium). $\mathrm{NaBD}_{4}(500 \mathrm{mg}, 12 \mathrm{mmol})$ was added and cooled in an ice bath. A solution of $\mathrm{I}_{2}$ $(2.03 \mathrm{~g}, 8.0 \mathrm{mmol})$ in THF $(10 \mathrm{~mL})$ was added dropwise over $30 \mathrm{~min}$ with vigorous evolution of hydrogen. After $\mathrm{H}_{2}$ evolution had ceased, the flask was heated to reflux for $12 \mathrm{~h}$ and then cooled to room temperature, diluted with methanol until the solid disappeared. After stirring $30 \mathrm{~min}$, the solvent was removed by rotary evaporation leaving a white paste which was dissolved by addition of $20 \%$ aqueous $\mathrm{KOH}(20 \mathrm{~mL})$. The solution was stirred for $4 \mathrm{~h}$ and extracted with $\mathrm{CH}_{2} \mathrm{Cl}_{2}(30 \mathrm{~mL} \times 2)$. The combined organic extracts were washed with brine, dried over anhydrous $\mathrm{MgSO}_{4}$, filtered, and concentrated under vacuum. The residue deuterated benzyl alcohol was used in the next step without purification.

A $100 \mathrm{~mL}$ round-bottom flask was charged with the residue deuterated benzyl alcohol $\mathrm{CH}_{3} \mathrm{COONa}(8$ mol \%), $4 \AA \mathrm{MS}$ and $\mathrm{CH}_{2} \mathrm{Cl}_{2}(20 \mathrm{~mL}), \mathrm{PCC}$ (1.5 equiv) was added slowly to a stirred solution. After addition completed, the reaction was stirred for another $3 \mathrm{~h}$. Then the reaction was filtrated by Celite. The organic extracts was concentrated under vacuum and purified by silica gel chromatography to give the deuterated benzaldehyde.

Deuterated benzaldehyde was dissolved in ethanol $(20 \mathrm{~mL})$. Then aqueous $\mathrm{NaOH}$ (2.0 equiv) solution was added dropwise followed by slow addition of the corresponding acetophenone. The reaction mixture was stirred at room temperature until the complete consumption of the starting materials (TLC). After completion, the reaction mixture was diluted with water and the precipitate was collected by filtration and washed with water and $\mathrm{EtOH}$, then $\alpha, \beta$-unsaturated deuterated carbonyl compound was obtained.

The $\alpha, \beta$-unsaturated deuterated carbonyl and $\mathrm{Pd} / \mathrm{C}$ were weighed directly into a Schlenk tube, dried under vacuum for $15 \mathrm{~min}$ and charged with $\mathrm{H}_{2}$. Then $\mathrm{EtOH}(10 \mathrm{~mL})$ was added and stirred for $24 \mathrm{~h}$. After completion, the reaction mixture was filtered, concentrated under vacuum and purified by silica gel chromatography to give the $\mathbf{2 a}-d^{3} .{ }^{1} \mathbf{H} \mathbf{N M R}\left(400 \mathrm{MHz}, \mathrm{CDCl}_{3}\right) \delta 7.97-7.94(\mathrm{~m}, 2 \mathrm{H}), 7.55(\mathrm{tt}, J=7.4,1.4 \mathrm{~Hz}$, 1H), 7.47-7.43 (m, 2H), 7.32-7.25 (m, 4H), $7.20(\mathrm{tt}, J=7.0,1.8 \mathrm{~Hz}, 1 \mathrm{H}), 3.29$ (d, $J=7.6 \mathrm{~Hz}, 2 \mathrm{H}), 3.05$ $(\mathrm{t}, J=7.6 \mathrm{~Hz}, 1 \mathrm{H}) .{ }^{35}$ 

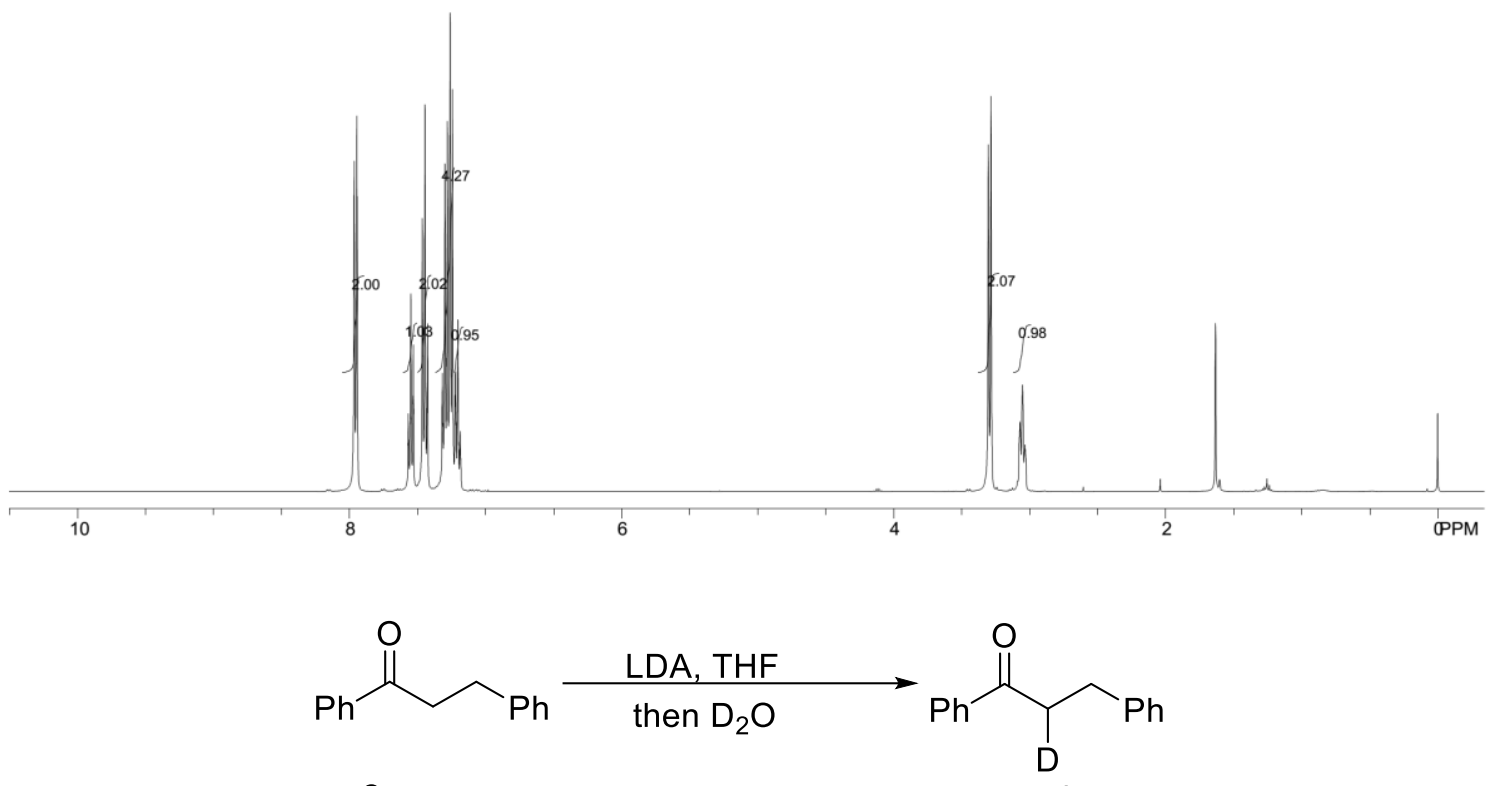

$2 a$

$2 \mathrm{a}-d^{2}$

2a $(0.5 \mathrm{mmol})$ was weighed directly into a Schlenk tube and dried under vacuum for $15 \mathrm{~min}$. Then solvent (THF, $1 \mathrm{~mL}$ ) was added and stirred under $-78^{\circ} \mathrm{C}$. LDA was added subsequently and stirred, After $15 \mathrm{~min}$, the reaction was allowed to warm to r.t. and stirred for additional $2 \mathrm{~h}$. Then the reaction was quenched by adding $\mathrm{D}_{2} \mathrm{O}$ and extracted with $\mathrm{Et}_{2} \mathrm{O}$. The combined organic extracts were washed with brine, dried over anhydrous $\mathrm{MgSO}_{4}$, filtered, concentrated under vacuum and purified by silica gel chromatography to give the $\mathbf{2 a}-d^{2} .{ }^{1} \mathbf{H}$ NMR $\left(400 \mathrm{MHz}, \mathrm{CDCl}_{3}\right) \delta 7.97-7.95(\mathrm{~m}, 2 \mathrm{H}), 7.55(\mathrm{tt}, J=7.4,1.4 \mathrm{~Hz}$, 1H), 7.45-7.43 (m, 2H), 7.32-7.18 (m, 5H), 3.32-3.26 (m, 1.25H), $3.07(\mathrm{t}, J=7.4 \mathrm{~Hz}, 2 \mathrm{H}){ }^{35}$ 

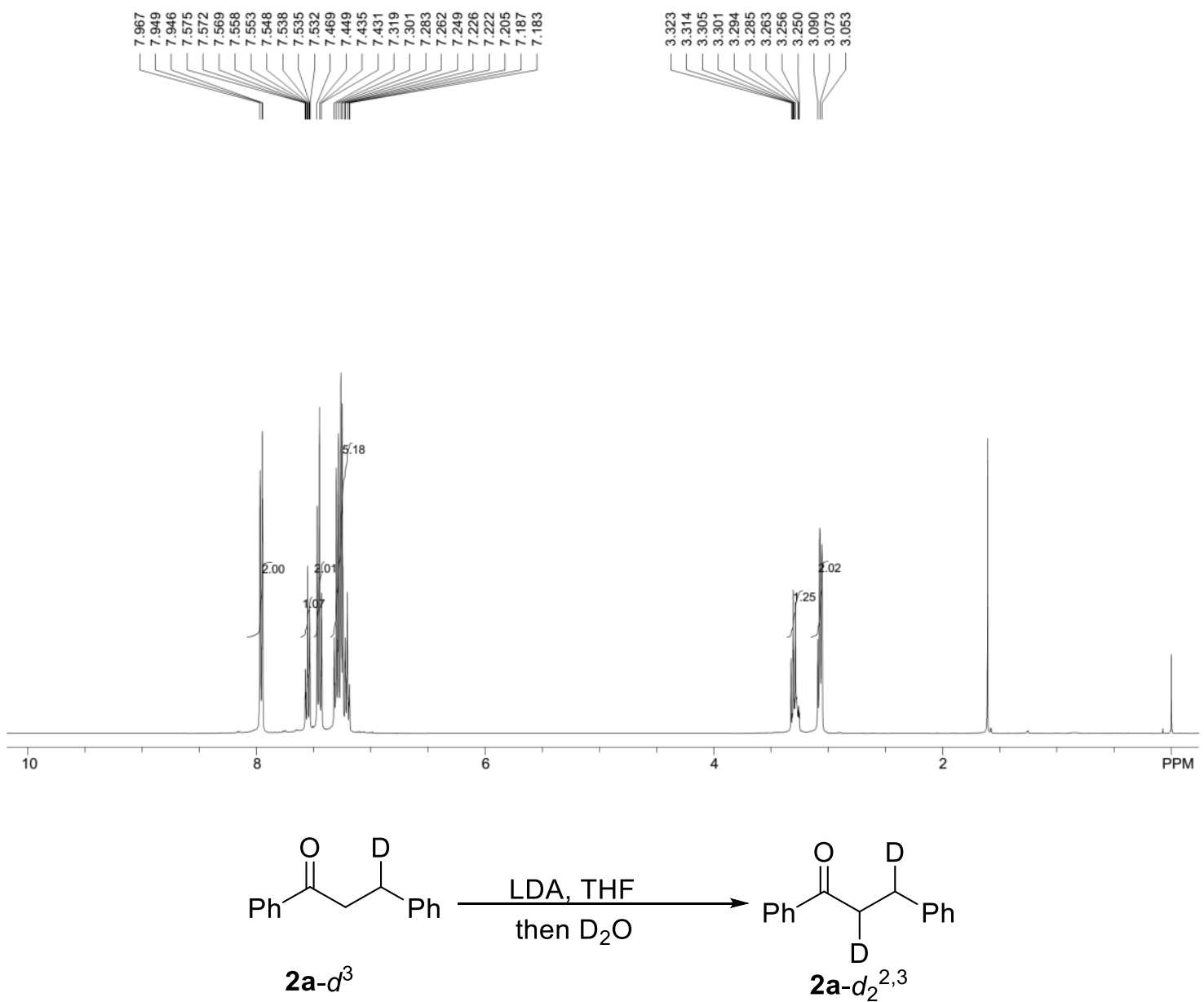

2a- $\mathrm{d}^{3}(0.5 \mathrm{mmol})$ was weighed directly into a Schlenk tube and dried under vacuum for $15 \mathrm{~min}$. Then solvent (THF, $1 \mathrm{~mL}$ ) was added and stirred under $-78^{\circ} \mathrm{C}$. LDA was added subsequently and stirred, After $15 \mathrm{~min}$, the reaction was allowed to warm to r.t. and stirred for additional $2 \mathrm{~h}$. Then the reaction was quenched by adding $\mathrm{D}_{2} \mathrm{O}$ and extracted with $\mathrm{Et}_{2} \mathrm{O}$. The combined organic extracts were washed with brine, dried over anhydrous $\mathrm{MgSO}_{4}$, filtered, concentrated under vacuum and purified by silica gel chromatography to give the $\mathbf{2 a}-d_{2}{ }^{2,3} .{ }^{1} \mathbf{H}$ NMR $\left(400 \mathrm{MHz}, \mathrm{CDCl}_{3}\right) \delta$ 7.97-7.95 (m, 2H), 7.55 (tt, $J=7.4,1.4$ $\mathrm{Hz}, 1 \mathrm{H}), 7.47-7.43(\mathrm{~m}, 2 \mathrm{H}), 7.32-7.18(\mathrm{~m}, 5 \mathrm{H}), 3.31-3.26(\mathrm{~m}, 1.23 \mathrm{H}), 3.05(\mathrm{t}, J=6.8 \mathrm{~Hz}, 1 \mathrm{H}){ }^{36}$ 


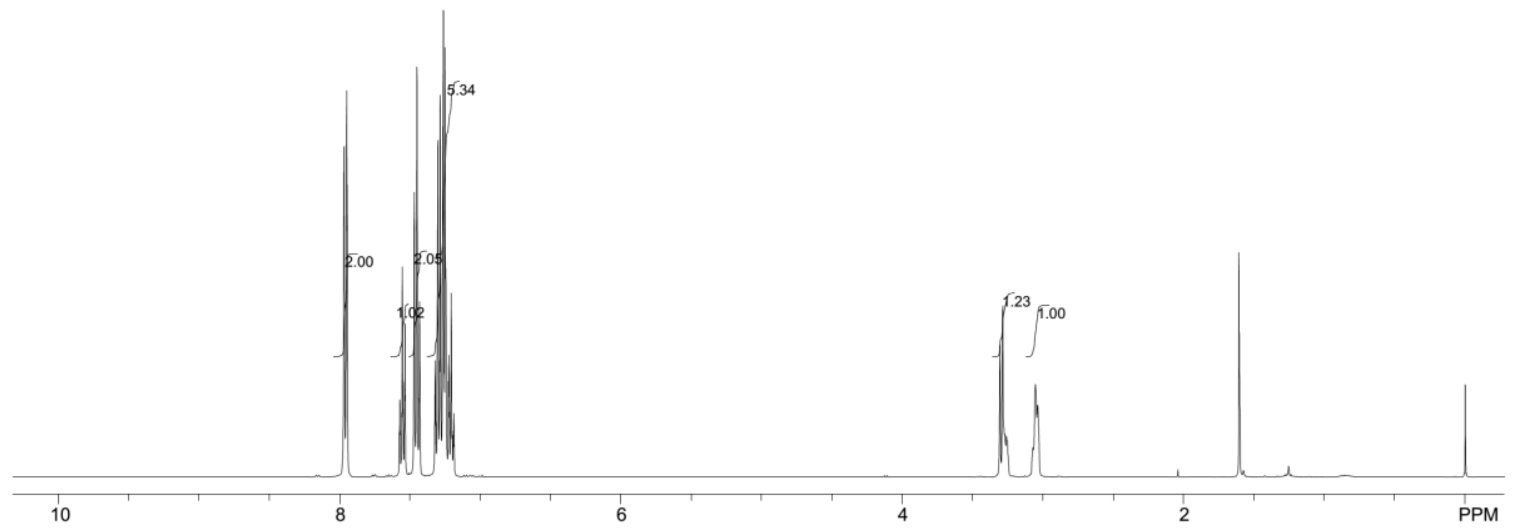

\subsubsection{Deuterium Labeling Experiments (Scheme 3)}

1a-d $(0.5 \mathrm{mmol})$ was weighed directly into a Schlenk tube and dried under vacuum for $15 \mathrm{~min}$. Then solvent (toluene, $1 \mathrm{~mL}$ ) was added and stirred. ${ }^{t} \mathrm{BuONa}(5 \mathrm{~mol} \%), 1,10$-phenanthroline $(5 \mathrm{~mol} \%)$ were then added and stirred at $90{ }^{\circ} \mathrm{C}$. The reaction was monitored by TLC. The mixture was purified by silica gel chromatography directly without further handling to give the desired product. 


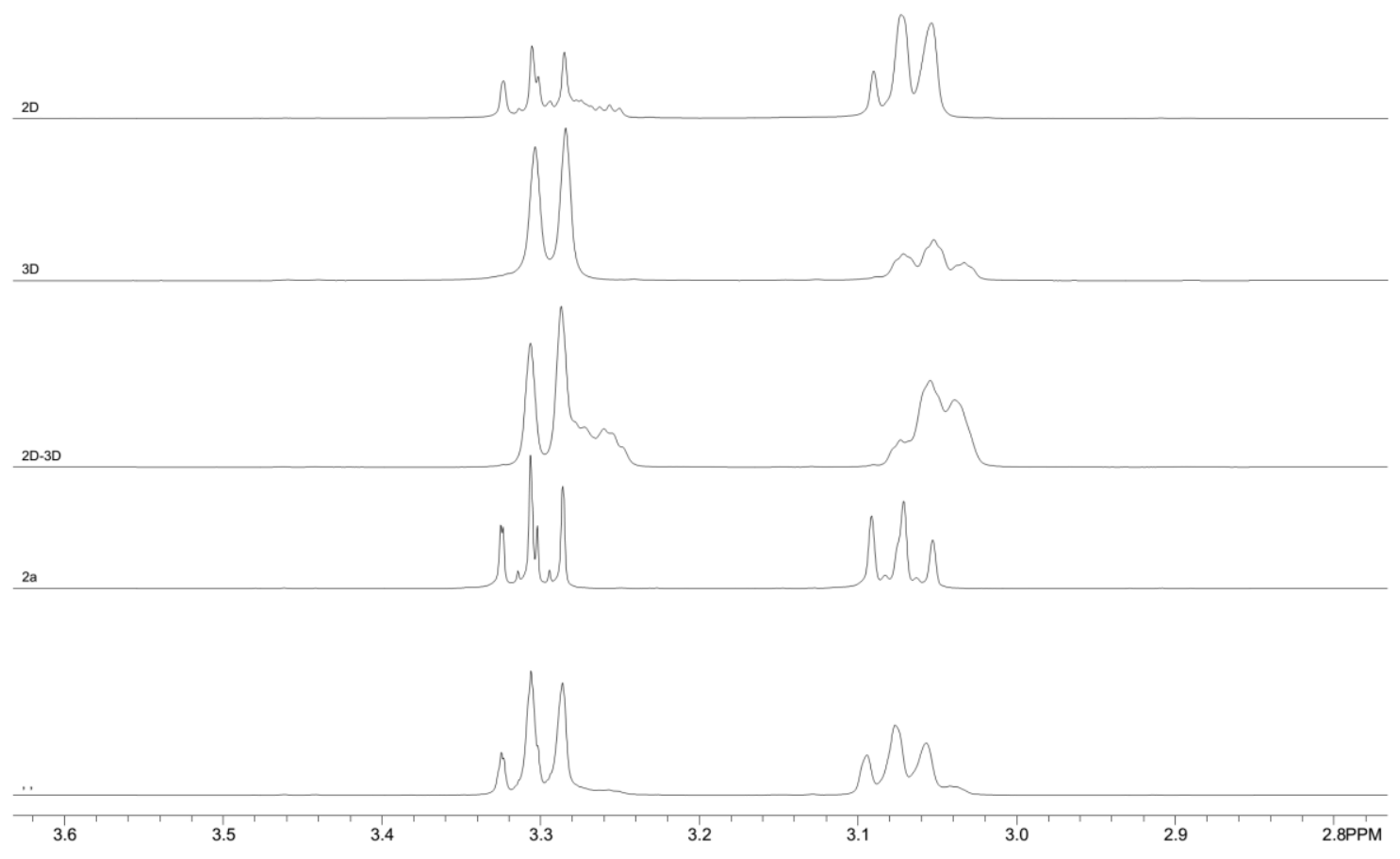

1a- $d(0.5 \mathrm{mmol})$ was weighed directly into a Schlenk tube and dried under vacuum for $15 \mathrm{~min}$. Then solvent (toluene, $1 \mathrm{~mL}$ ) was added and stirred. ${ }^{t} \mathrm{BuOK}$ (200 mol \%), then added and stirred at room temperature for 2 hours. The mixture was purified by silica gel chromatography directly without further handling to give the product.

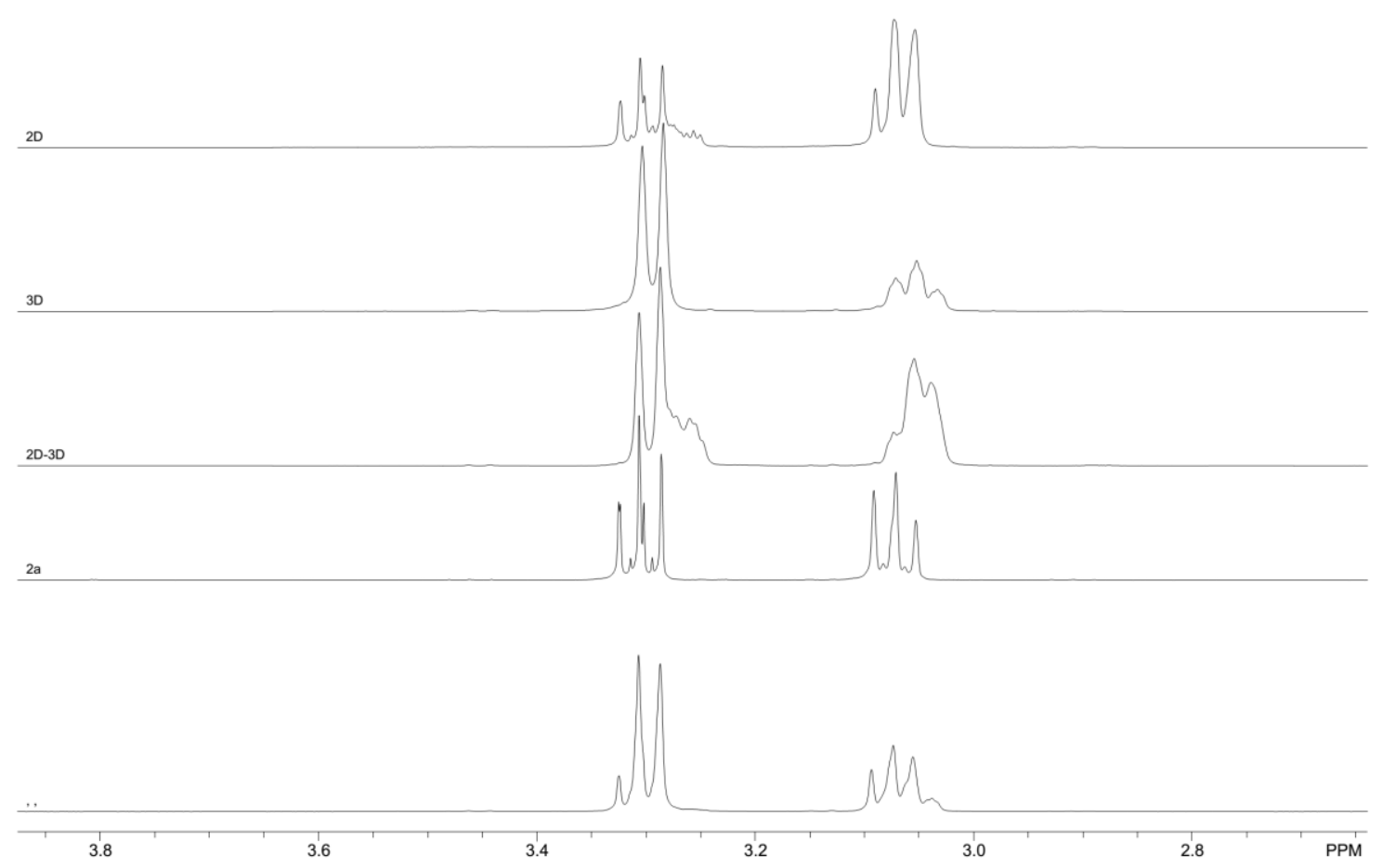

1a $(0.5 \mathrm{mmol})$ was weighed directly into a Schlenk tube and dried under vacuum for $15 \mathrm{~min}$. Then solvent (toluene, $1 \mathrm{~mL}$ ) was added and stirred. $\mathrm{D}_{2} \mathrm{O}(100 \mathrm{~mol} \%), 1,10$-phenanthroline $(10 \mathrm{~mol} \%)$ and 
${ }^{t} \mathrm{BuONa}(110 \mathrm{~mol} \%)$ then added and stirred at room temperature for 1.5 hours. The mixture was purified by silica gel chromatography directly without further handling to give the product.

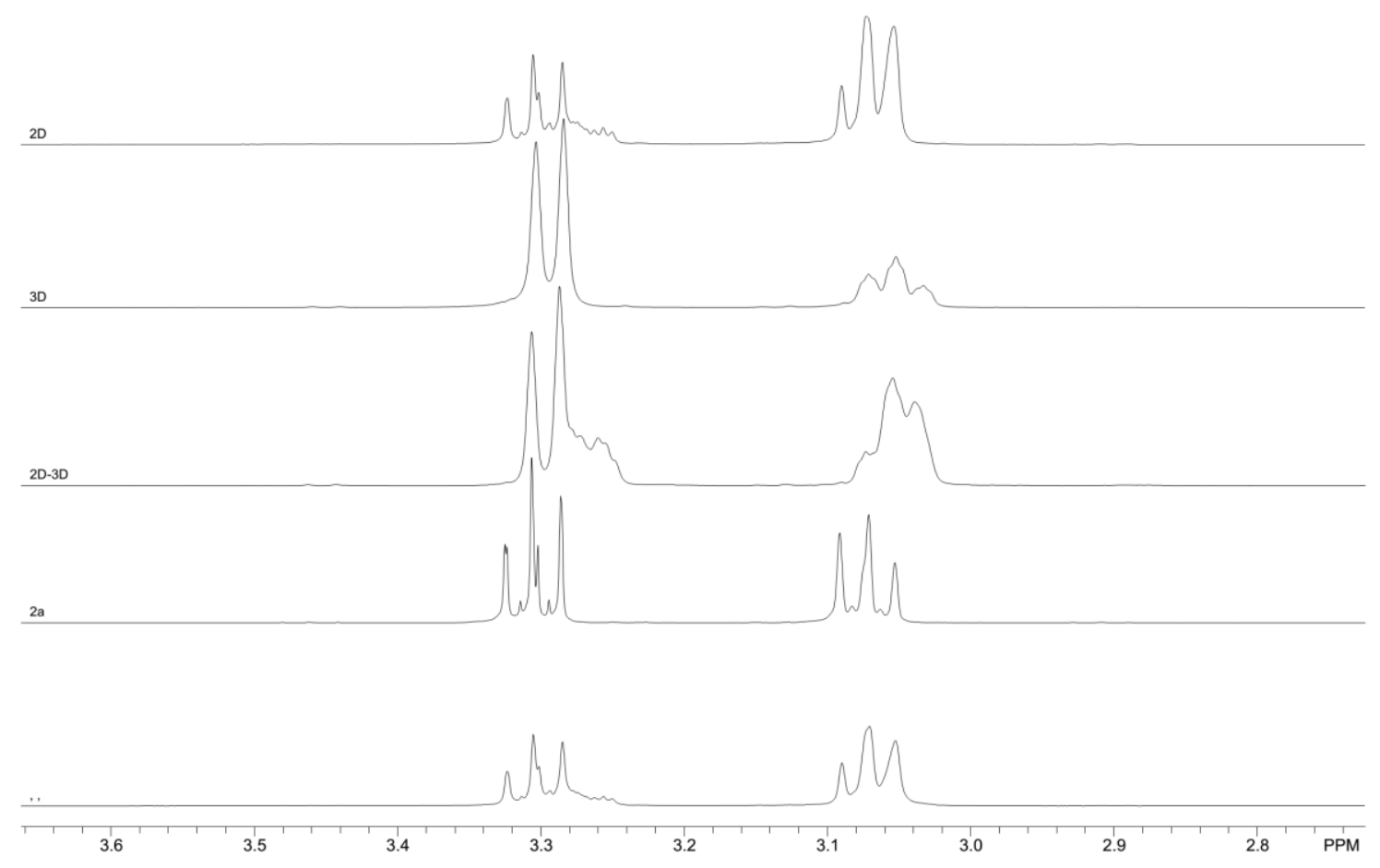

$1 \mathrm{a}(0.5 \mathrm{mmol})$ was weighed directly into a Schlenk tube and dried under vacuum for $15 \mathrm{~min}$. Then solvent (toluene, $1 \mathrm{~mL}$ ) was added and stirred. $\mathrm{D}_{2} \mathrm{O}(100 \mathrm{~mol} \%)$ and $t \mathrm{BuONa}(300 \mathrm{~mol} \%)$ then added and stirred at room temperature for 1.5 hours. The mixture was purified by silica gel chromatography directly without further handling to give the product. 


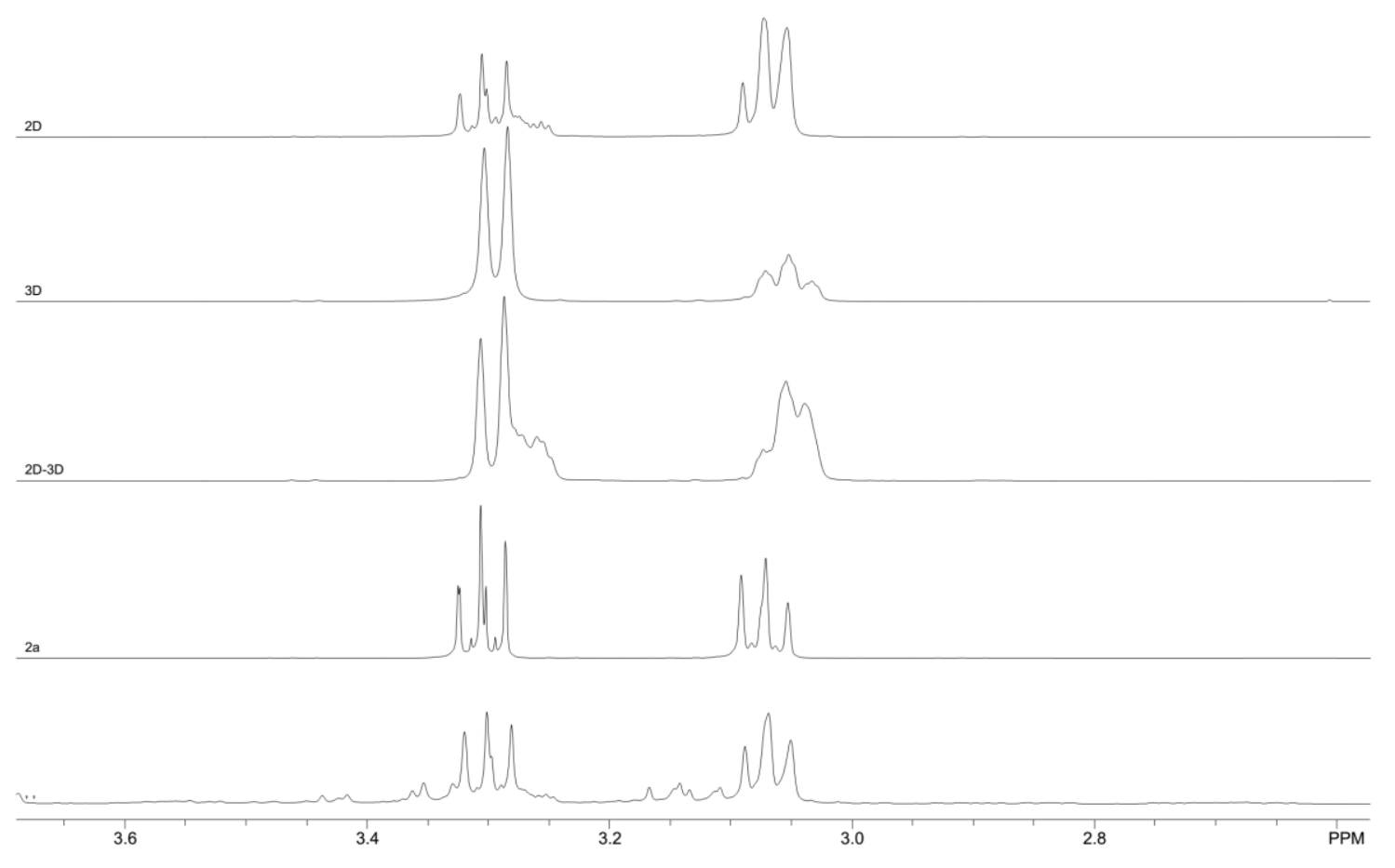

\subsubsection{Kinetic isotopic effect}

Two parallel reactions with 1a and 1a- $d$ were ran at same time. 1a or 1a- $d(0.5 \mathrm{mmol})$ was weighed directly into a Schlenk tube and dried under vacuum for $15 \mathrm{~min}$. Then solvent (toluene, $1 \mathrm{~mL}$ ) was added and stirred. 1,10-phenanthroline was then added and 1,3-dimethoxybenzene (22 $\mu \mathrm{L})$ was added as internal standard, the resulting mixture was stirred at $90{ }^{\circ} \mathrm{C}$. The sample mixture was used to collect ${ }^{1} \mathrm{H}$ NMR (400 $\left.\mathrm{MHz}{ }^{1} \mathrm{H} \mathrm{NMR}\right)$ data. The recovery of $\mathbf{1 a} / \mathbf{1 a}-d$ was determined by 1,3-dimethoxybenzene from the ${ }^{1} \mathrm{H}$ NMR spectra.

\subsection{Kinetic study}

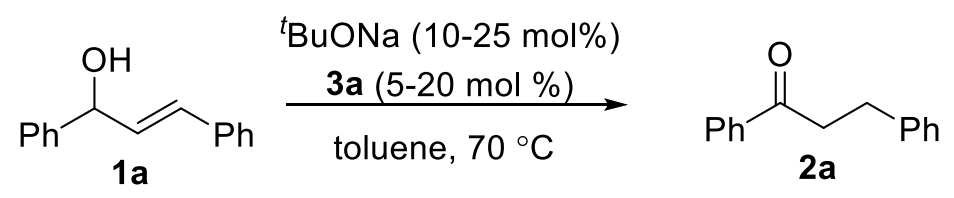

All data was collected on $400 \mathrm{MHz}{ }^{1} \mathrm{H}$ NMR using 1,3-dimethoxybenzene as the internal standard. The initial rates were calculated as the slopes of time zero on the conversion curves of $[(E)$-1,3-diphenylprop2-en-1-ol] against time.

Reaction conditions (initial): (E)-1,3-diphenylprop-2-en-1-ol(1.0 mol, $210.26 \mathrm{mg})$ was weighed into a Schlenk tube. After dried in vacuo for $15 \mathrm{~min}$, then $2 \mathrm{~mL}$ toluene was added. Under stirring, ${ }^{t} \mathrm{BuONa}(2.5-$ $12.5 \mathrm{~mol} \%, 2.5-12.5 \mathrm{mg})$ and 3a (5-20 mol \%, 4.5-36 mg) were added. 1,3-Dimethoxybenzene (22 $\mu \mathrm{L})$ 
was added as internal standard and the mixture was stirred at $70{ }^{\circ} \mathrm{C}, 535 \mathrm{rpm}$. Timing begins with adding $t \mathrm{BuONa}$, at regular intervals $40 \mu \mathrm{L}$ mixture solution was sampled from the Schlenk tube. The sample mixture was used to collect ${ }^{1} \mathrm{H}$ NMR (400 MHz ${ }^{1} \mathrm{H}$ NMR) data. The recovery of (E)-1,3-diphenylprop-2en-1-ol was determined by 1,3-dimethoxybenzene from the ${ }^{1} \mathrm{H}$ NMR spectra.

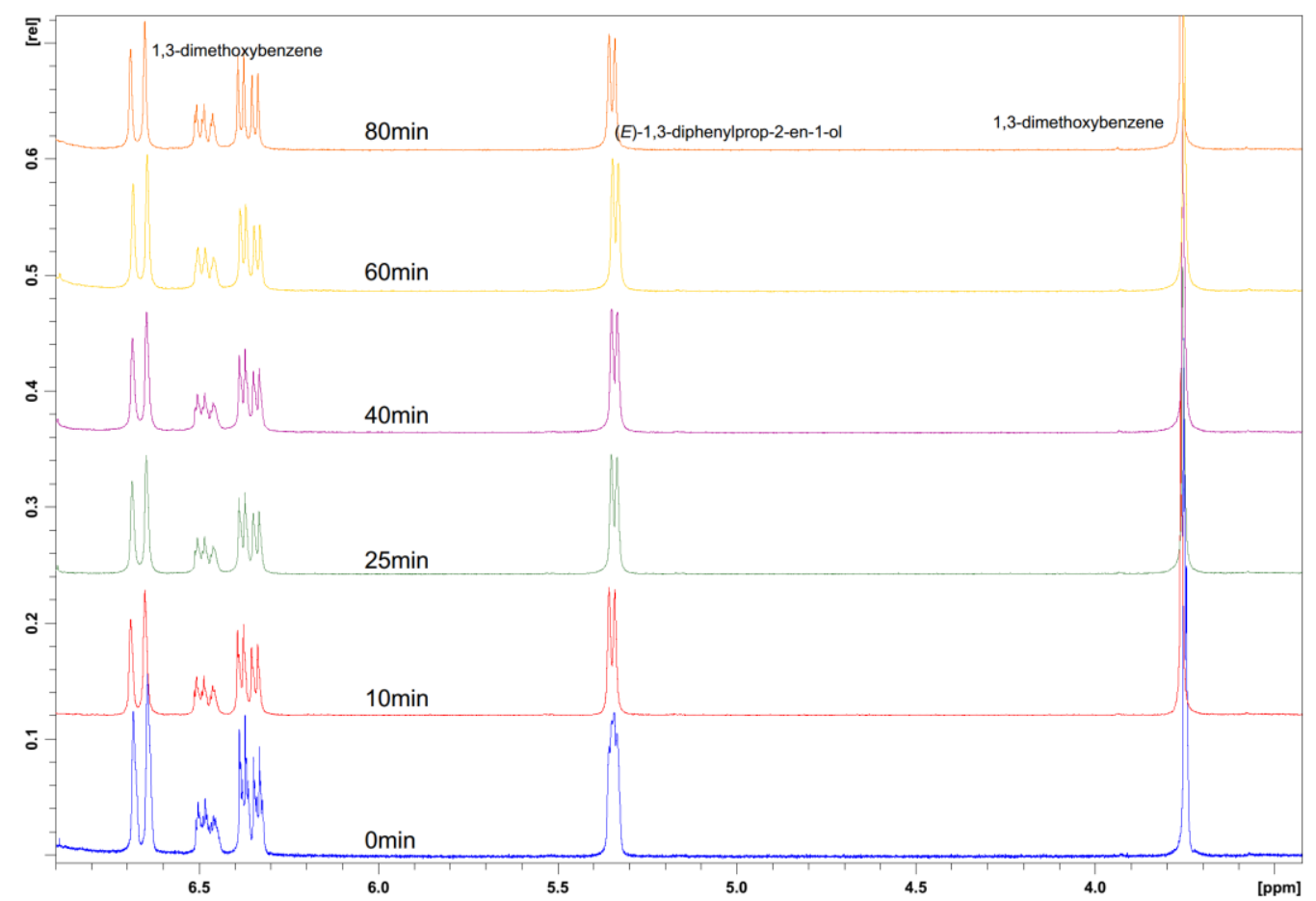

Figure S1. ${ }^{1} \mathrm{H}$ NMR for $[(E)-1,3$-diphenylprop-2-en-1-ol $]=0.5 \mathrm{M}(1 \mathrm{mmol}, 210.26 \mathrm{mg}),{ }^{t} \mathrm{BuONa}(2.5$ $\mathrm{mol} \%, 2.5 \mathrm{mg})$ and 3a $(20 \mathrm{~mol} \%, 36 \mathrm{mg})$, toluene $(2 \mathrm{~mL}), 70{ }^{\circ} \mathrm{C}, 535 \mathrm{rpm}, 1,3$-dimethoxybenzene $(22$ $\mu \mathrm{L})$ as internal standard.

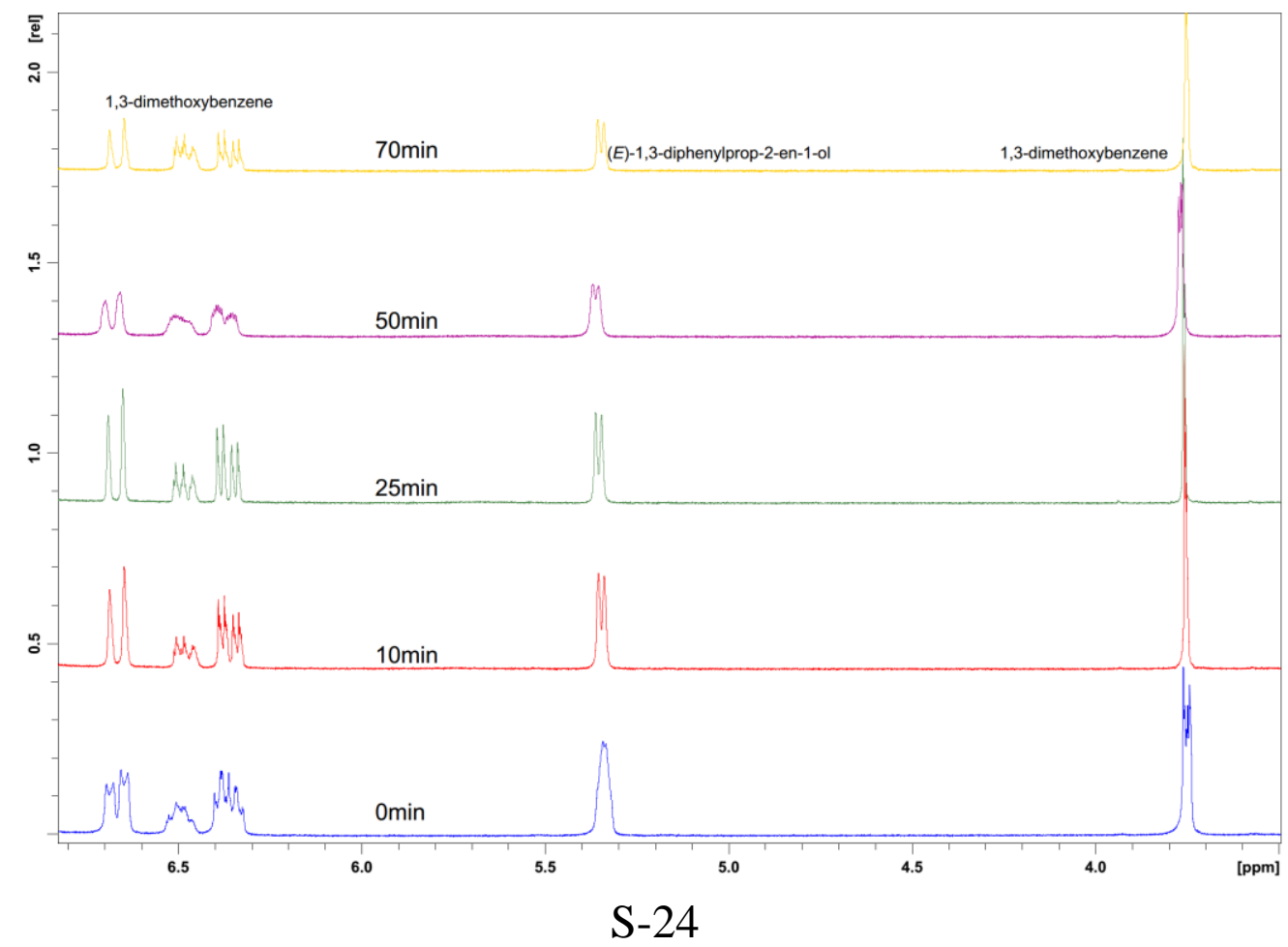


Figure S2. ${ }^{1} \mathrm{H}$ NMR for $[(E)-1,3$-diphenylprop-2-en-1-ol $]=0.5 \mathrm{M}(1 \mathrm{mmol}, 210.26 \mathrm{mg}),{ }^{t} \mathrm{BuONa}(5$ $\mathrm{mol} \%, 5 \mathrm{mg})$ and 3a $(20 \mathrm{~mol} \%, 36 \mathrm{mg})$, toluene $(2 \mathrm{~mL}), 70{ }^{\circ} \mathrm{C}, 535 \mathrm{rpm}, 1,3$-dimethoxybenzene $(22 \mu \mathrm{L})$ as internal standard.

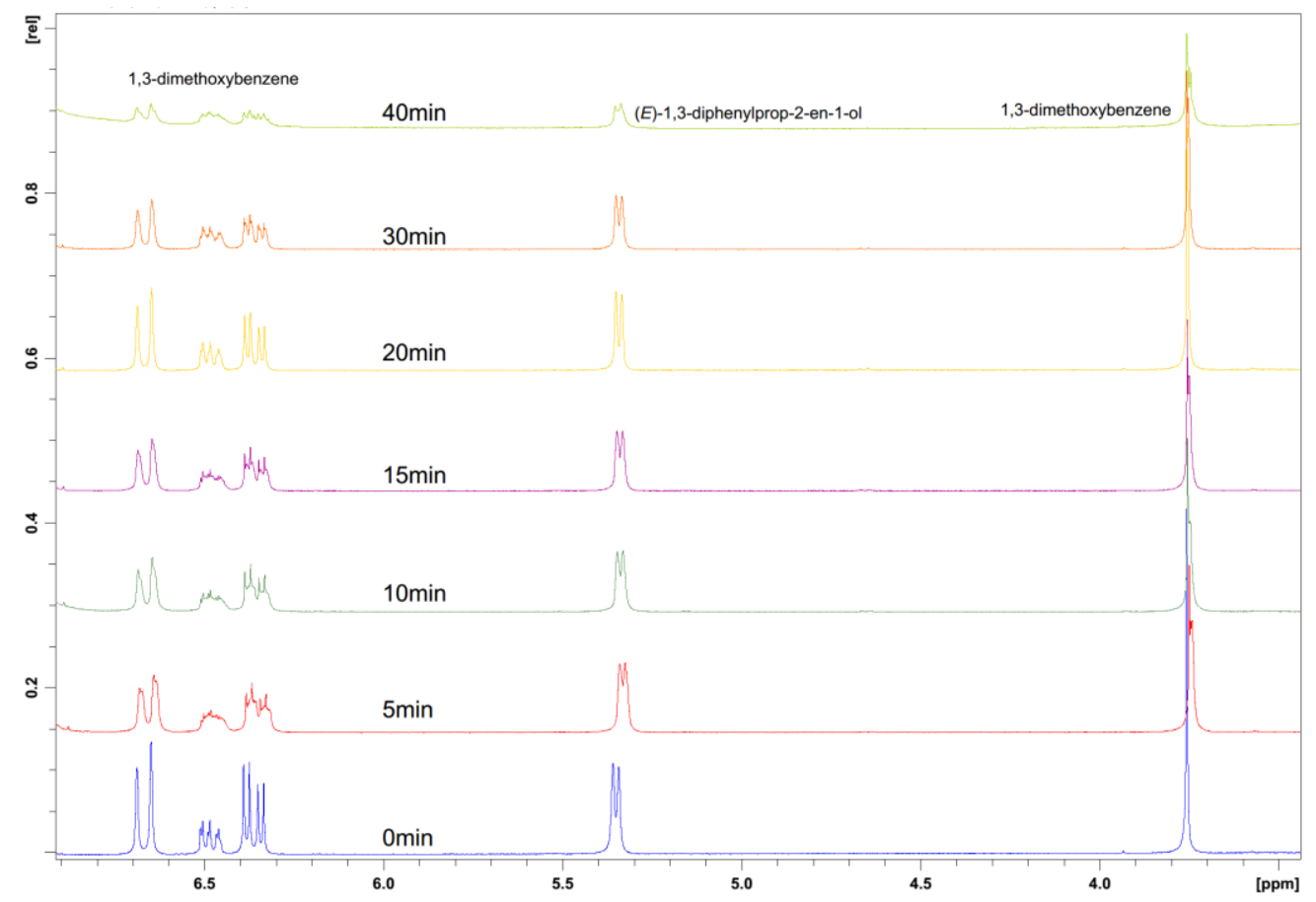

Figure S3. ${ }^{1} \mathrm{H}$ NMR for $[(E)-1,3$-diphenylprop-2-en-1-ol $]=0.5 \mathrm{M}(1 \mathrm{mmol}, 210.26 \mathrm{mg}),{ }^{t} \mathrm{BuONa}(7.5$ $\mathrm{mol} \%, 7.5 \mathrm{mg})$ and $\mathbf{3 a}(20 \mathrm{~mol} \%, 36 \mathrm{mg})$, toluene $(2 \mathrm{~mL}), 70{ }^{\circ} \mathrm{C}, 535 \mathrm{rpm}, 1,3$-dimethoxybenzene $(22$ $\mu \mathrm{L})$ as internal standard.

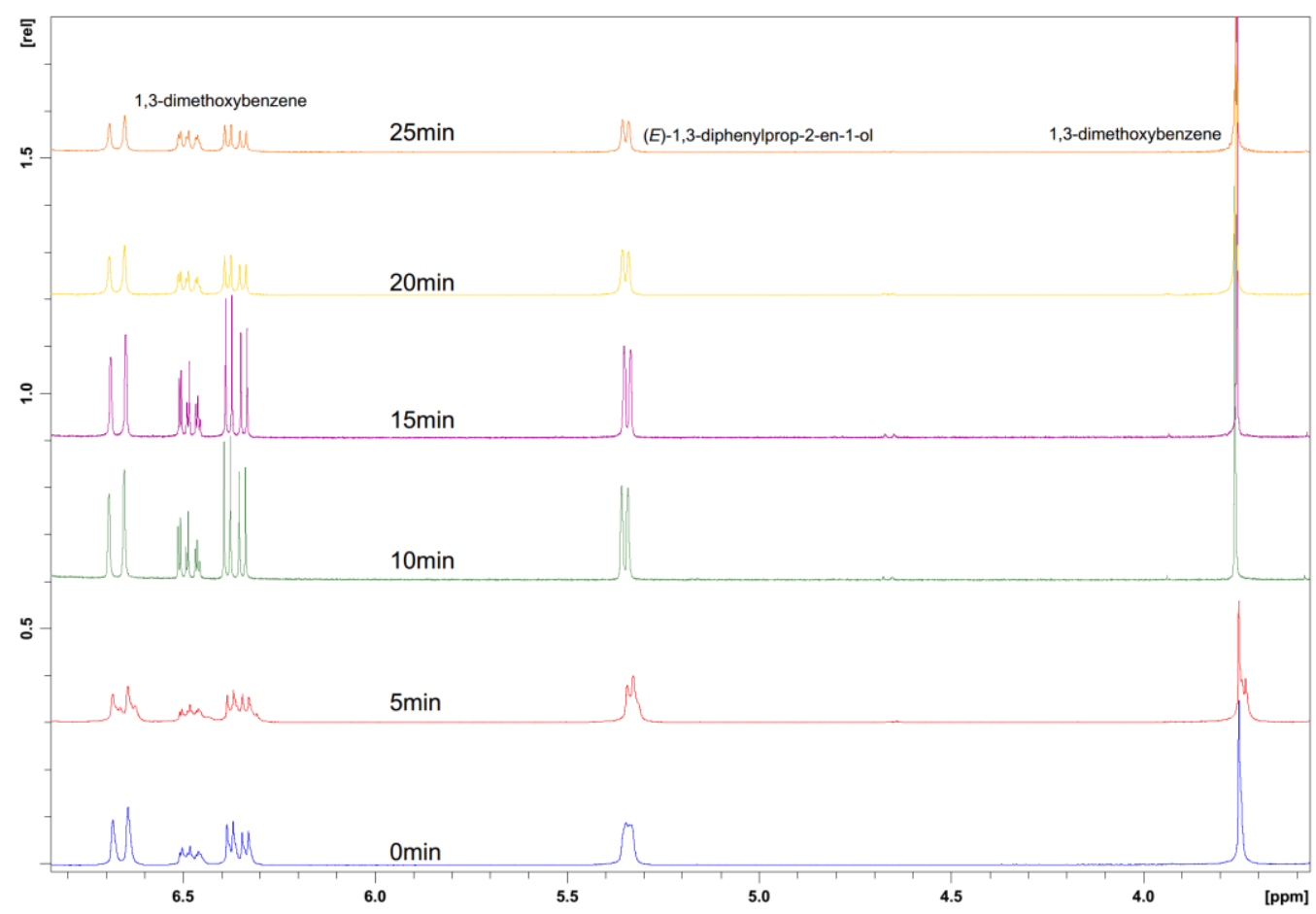

Figure S4. ${ }^{1} \mathrm{H}$ NMR for $[(E)-1,3$-diphenylprop-2-en-1-ol $]=0.5 \mathrm{M}(1 \mathrm{mmol}, 210.26 \mathrm{mg}),{ }^{t} \mathrm{BuONa}(10$ $\mathrm{mol} \%, 10 \mathrm{mg})$ and $3 \mathrm{a}(20 \mathrm{~mol} \%, 36 \mathrm{mg})$, toluene $(2 \mathrm{~mL}), 70{ }^{\circ} \mathrm{C}, 535 \mathrm{rpm}, 1,3$-dimethoxybenzene $(22$ S-25 
$\mu \mathrm{L})$ as internal standard.

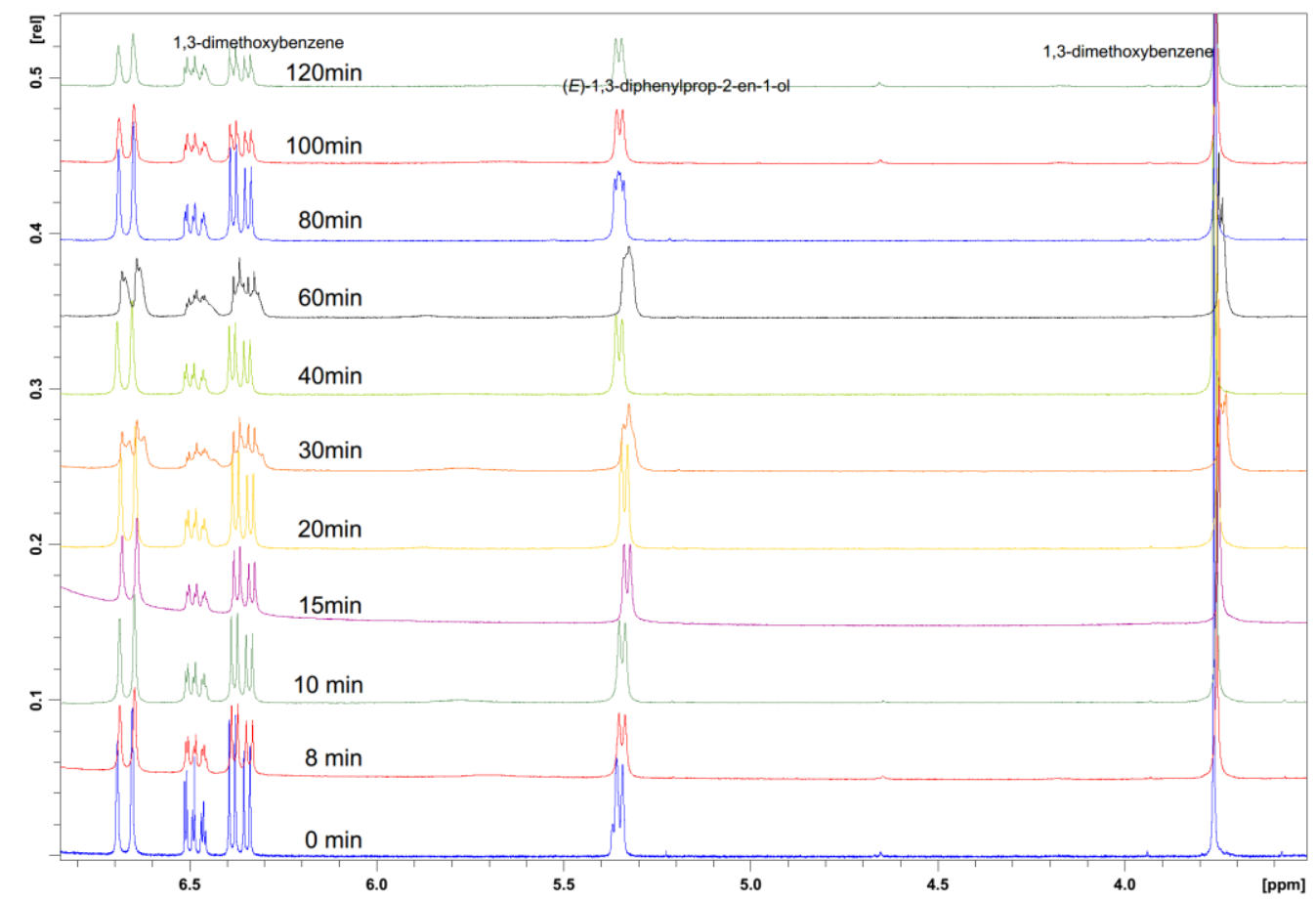

Figure S5. ${ }^{1} \mathrm{H}$ NMR for $[(E)-1,3$-diphenylprop-2-en-1-ol $]=0.5 \mathrm{M}(1 \mathrm{mmol}, 210.26 \mathrm{mg}),{ }^{t} \mathrm{BuONa}(10$ $\mathrm{mol} \%, 10 \mathrm{mg})$ and $3 \mathbf{a}(5 \mathrm{~mol} \%, 9 \mathrm{mg})$, toluene $(2 \mathrm{~mL}), 70{ }^{\circ} \mathrm{C}, 535 \mathrm{rpm}, 1,3$-dimethoxybenzene $(22 \mu \mathrm{L})$ as internal standard.

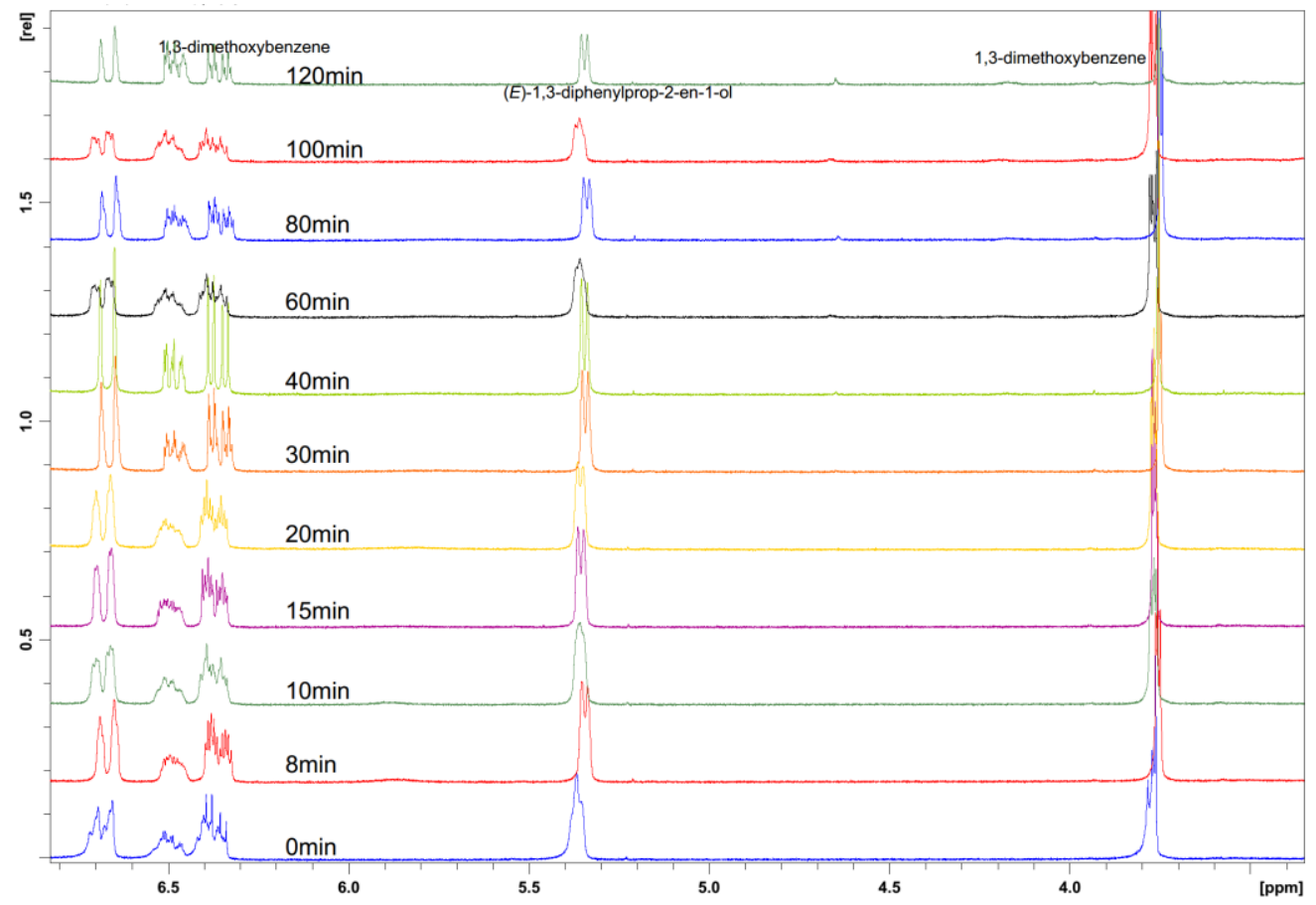

Figure S6. ${ }^{1} \mathrm{H}$ NMR for $\left[(E)-1,3\right.$-diphenylprop-2-en-1-ol] $=0.5 \mathrm{M}(1 \mathrm{mmol}, 210.26 \mathrm{mg}),{ }^{t} \mathrm{BuONa}(10$ $\mathrm{mol} \%, 10 \mathrm{mg})$ and $\mathbf{3 a}(7.5 \mathrm{~mol} \%, 13.5 \mathrm{mg})$, toluene $(2 \mathrm{~mL}), 70{ }^{\circ} \mathrm{C}, 535 \mathrm{rpm}, 1,3$-dimethoxybenzene $(22$ $\mu \mathrm{L})$ as internal standard. 


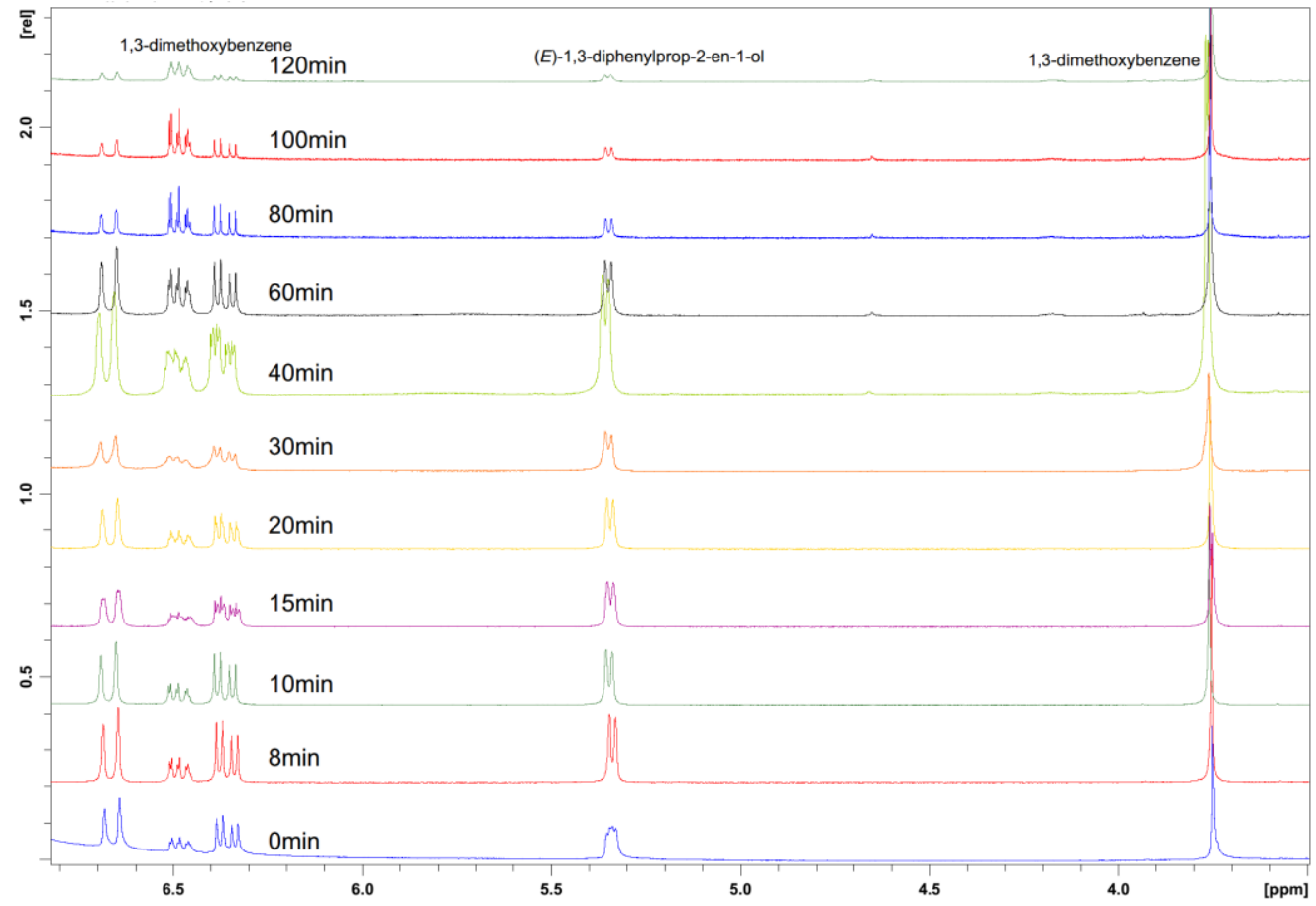

Figure S7. ${ }^{1} \mathrm{H}$ NMR for $[(E)-1,3-d i p h e n y l p r o p-2-e n-1-o l]=0.5 \mathrm{M}(1 \mathrm{mmol}, 210.26 \mathrm{mg}),{ }^{t} \mathrm{BuONa}(10$ $\mathrm{mol} \%, 10 \mathrm{mg})$ and 3a $(10 \mathrm{~mol} \%, 18 \mathrm{mg})$, toluene $(2 \mathrm{~mL}), 70{ }^{\circ} \mathrm{C}, 535 \mathrm{rpm}, 1,3$-dimethoxybenzene $(22$ $\mu \mathrm{L})$ as internal standard.

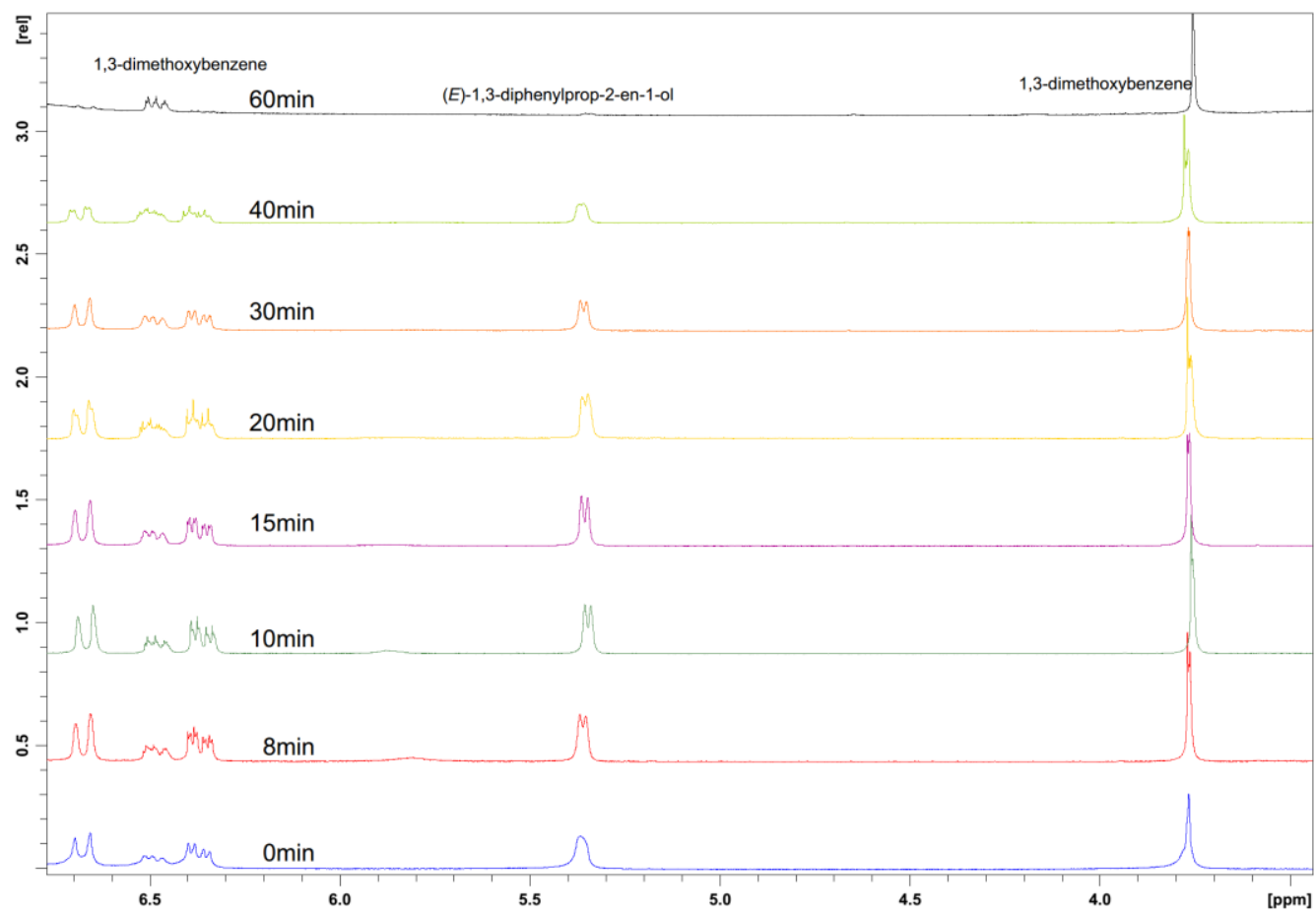

Figure S8. ${ }^{1} \mathrm{H}$ NMR for $[(E)-1,3$-diphenylprop-2-en-1-ol $]=0.5 \mathrm{M}(1 \mathrm{mmol}, 210.26 \mathrm{mg}),{ }^{t} \mathrm{BuONa}(10$ $\mathrm{mol} \%, 10 \mathrm{mg})$ and $\mathbf{3 a}(12.5 \mathrm{~mol} \%, 22.5 \mathrm{mg})$, toluene $(2 \mathrm{~mL}), 70{ }^{\circ} \mathrm{C}, 535 \mathrm{rpm}, 1,3$-dimethoxybenzene $(22 \mu \mathrm{L})$ as internal standard. 

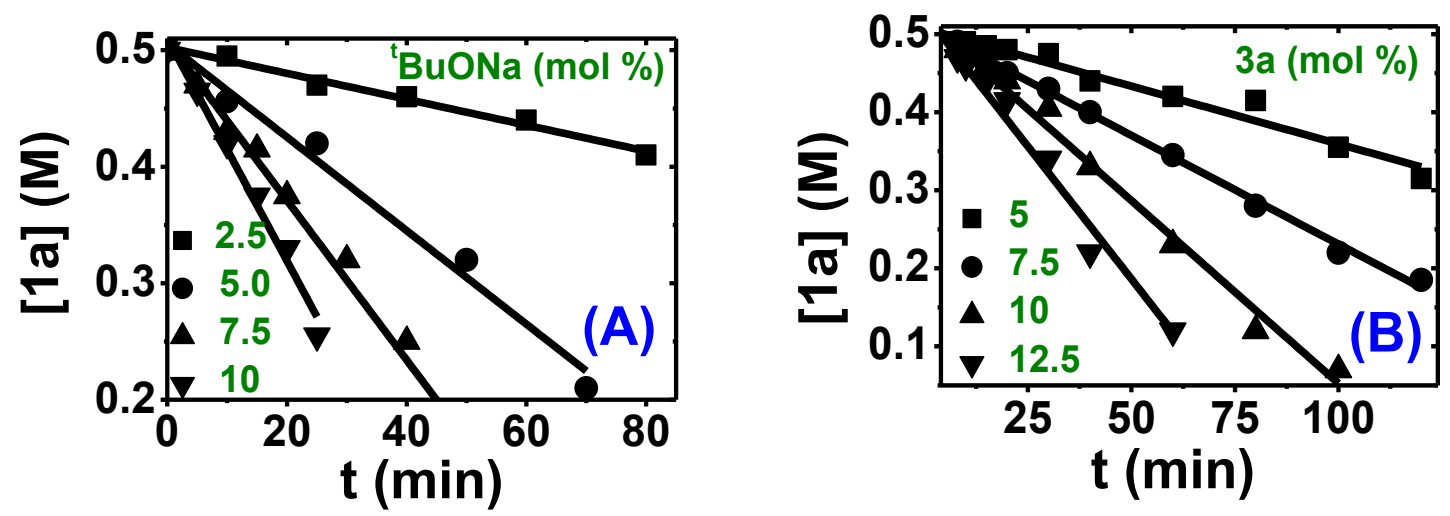

(A) Plots of [1a] v.s. time with different amounts of ${ }^{t} \mathrm{BuONa}$. (B) Plots of [1a] v.s. time with different amounts of $\mathbf{3 a}$.

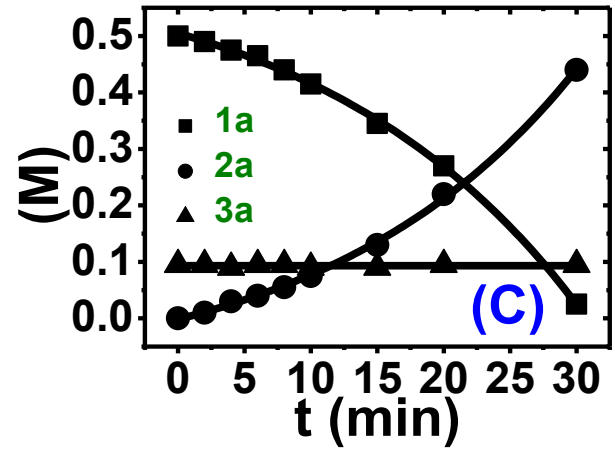

(C) Plots of [1a], [2a], [3a] v.s. time .

\section{EPR experiments and radical trapping experiments}

\subsubsection{EPR experiments for $3 \mathrm{a}-{ }^{t} \mathrm{BuONa}$ system}

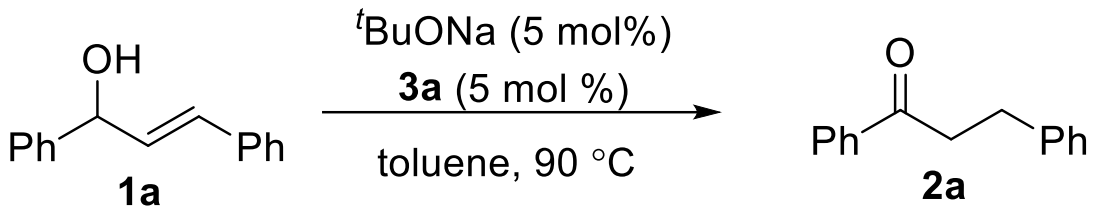

1 a $(0.5 \mathrm{mmol})$ was weighed directly into a Schlenk tube and dried under vacuum for $15 \mathrm{~min}$. Then solvent (toluene, $1 \mathrm{~mL}$ ) was added and stirred. ${ }^{t} \mathrm{BuONa}(5 \mathrm{~mol} \%, 2.5 \mathrm{mg}), 1,10$-phenanthroline $(5 \mathrm{~mol} \%$, $4.5 \mathrm{mg}$ ) were added and stirred at 90 for $1 \mathrm{~h}$. Then the reaction mixture was freezed in liquid nitrogen. The reaction mixture was analyzed by EPR at $-170 \mathrm{~K}$ (Spectra see article).

\subsubsection{EPR experiments for 2 equiv of ${ }^{t} \mathrm{BuOK}$}

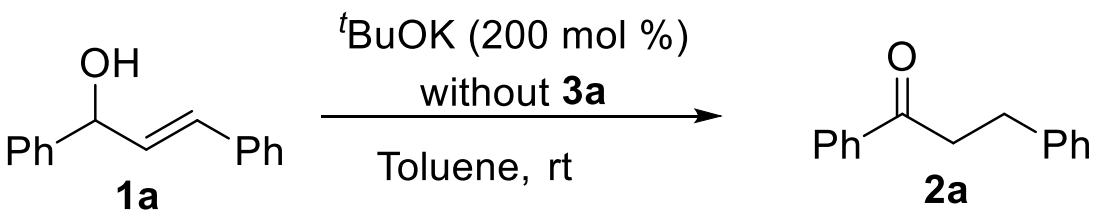


$1 \mathbf{a}(0.5 \mathrm{mmol})$ was weighed directly into a Schlenk tube and dried under vacuum for $15 \mathrm{~min}$. Then solvent (toluene, 1mL) was added and stirred. ${ }^{t} \mathrm{BuOK}(200 \mathrm{~mol} \%, 112.21 \mathrm{mg})$ was added and stirred at $\mathrm{rt}$. Then the reaction mixture was freezed in liquid nitrogen. The reaction mixture was analyzed by EPR at $-170 \mathrm{~K}$. The spectra illustrated below shows that radical can be seen in the reaction with 2 equiv of ${ }^{t} \mathrm{BuOK}$.

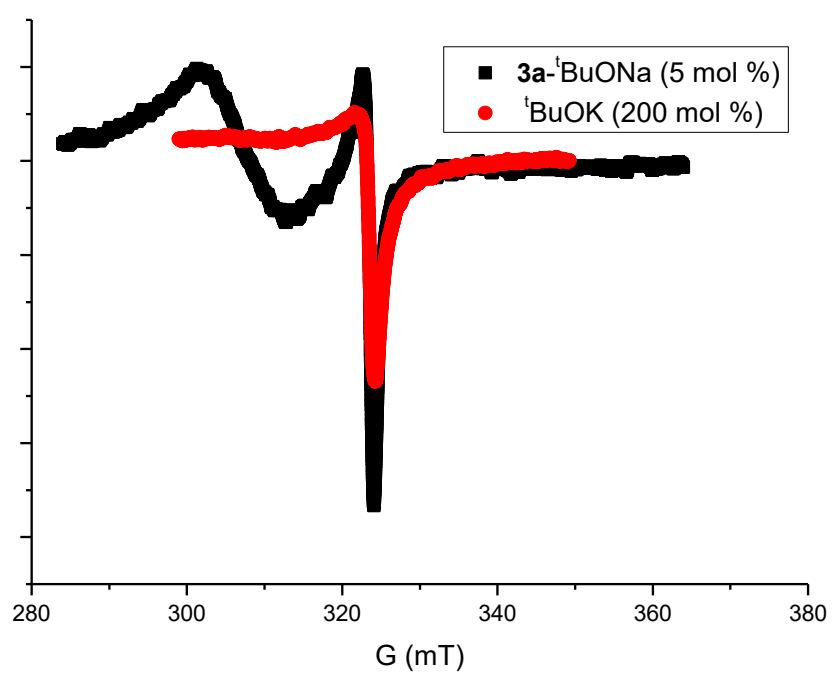

\subsubsection{Radical trapping experiments for $3 \mathrm{a}^{-}{ }^{\mathrm{t}} \mathrm{BuONa}$ system}

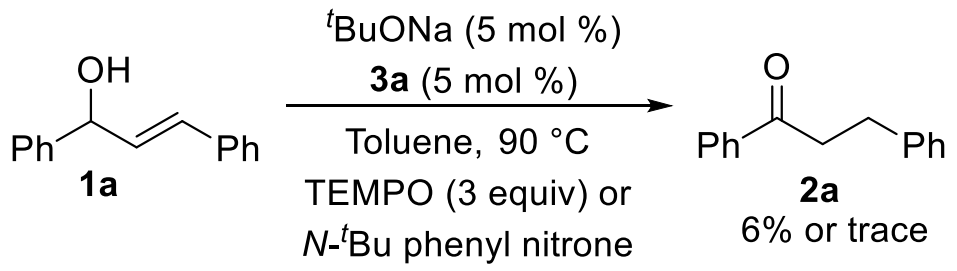

1 a $(0.5 \mathrm{mmol})$ was weighed directly into a Schlenk tube and dried under vacuum for $15 \mathrm{~min}$. Then solvent (toluene $1 \mathrm{~mL}$ ), TEMPO or $N^{-}{ }^{t} \mathrm{Bu}$ phenyl nitrone (3.0 equiv) was added and stirred. ${ }^{t} \mathrm{BuONa}(5$ $\mathrm{mol} \%, 2.5 \mathrm{mg}), 1,10$-phenanthroline $(5 \mathrm{~mol} \%, 4.5 \mathrm{mg}$ ) were added and stirred at 90 for $2 \mathrm{~h}$. The reaction mixture was directly examined on ${ }^{1} \mathrm{H}$ NMR spectrometer to determine the conversion and selectivity by using 1,4-dioxane $(10.7 \mu \mathrm{L})$ and 1,3-dimethoxybenzene $(22 \mu \mathrm{L})$ as internal standards.

\subsubsection{Radical trapping experiments for 2 equiv of ${ }^{t} \mathrm{BuOK}$}

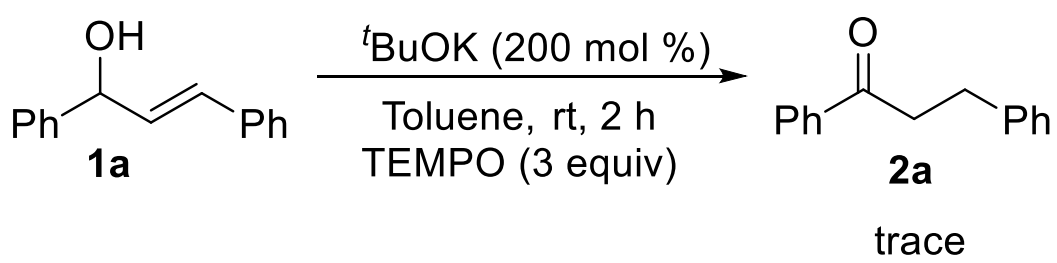

$1 \mathbf{a}(0.5 \mathrm{mmol})$ was weighed directly into a Schlenk tube and dried under vacuum for $15 \mathrm{~min}$. Then 
solvent (toluene $1 \mathrm{~mL}$ ), TEMPO (3.0 equiv) was added and stirred. ${ }^{t} \mathrm{BuOK}$ (200 mol \%, $112.21 \mathrm{mg}$ ) was added and stirred at $\mathrm{rt}$ for $2 \mathrm{~h}$. The reaction mixture was directly examined on ${ }^{1} \mathrm{H}$ NMR spectrometer to determine the conversion and selectivity by using 1,4-dioxane $(10.7 \mu \mathrm{L})$ and 1,3-dimethoxybenzene $(22$ $\mu \mathrm{L})$ as internal standards. There is no $\mathbf{2 a}$ observed and $\mathbf{1 a}$ was recivered. TEMPO was recovered without change. Therefore the stoichiometric ${ }^{t} \mathrm{BuOK}$ promoted isomerization of $\mathbf{1 a}$ probably is radical process as well.

\section{ICP-AES analysis (Table S4)}

${ }^{t} \mathrm{BuONa}$ was dissolved in aqua regia and ICP-AES was used to determine the trace metals and all suspected metals were not detected ( $\mathrm{Pd}, \mathrm{Ir}, \mathrm{Ru}, \mathrm{Ni}, \mathrm{Rh}, \mathrm{Pt}, \mathrm{Fe}, \mathrm{Co}, \mathrm{Os}$ ).

After the reaction mixture baked at $1173 \mathrm{~K}$, the residue was dissolved in aqua regia and ICP-AES was used to determine the trace metals and all suspected metals were not detected (Pd, Ir, $\mathrm{Ru}, \mathrm{Ni}, \mathrm{Rh}, \mathrm{Pt}, \mathrm{Fe}$, Co, Os).

\section{Table S4.}

\begin{tabular}{ccc}
\hline \multirow{2}{*}{ Metallic elements } & \multicolumn{2}{c}{ Content of elements $(\mu \mathrm{g} / \mathrm{g})$} \\
\cline { 2 - 3 } $\mathrm{Pd}$ & ${ }^{t} \mathrm{BuONa}$ & Reaction system \\
$\mathrm{n}$ Ir & 12.80 & N. D. \\
$\mathrm{Ru}$ & N. D. & N. D. \\
$\mathrm{Rh}$ & 0.30 & N. D. \\
$\mathrm{Fe}$ & N. D. & 0.025 \\
$\mathrm{Ni}$ & 80.0 & N. D. \\
$\mathrm{Co}$ & 6.7 & N. D. \\
$\mathrm{Os}$ & 6.9 & N. D. \\
\hline
\end{tabular}




\section{References}

(1) Liu, S.; Liebeskind, L. S. J. Am. Chem. Soc. 2008, 130, 6918.

(2) Fukuzawa, S.; Fujinami, T.; Yamauchi, S.; Sakai, S. J. Chem. Soc. Perkin. Trans. 1986, 1, 1929.

(3) Jautze, S.; Peters, R. Angew. Chem., Int. Ed. 2008, 47, 9284.

(4) Adam, J. S.; McLaughlin, M. G.; Reid, J. P.; Cook, M. J. Org. Biomol. Chem. 2013, 11, 7662.

(5) Akai, S.; Hanada, R.; Fujiwara, N.; Kita, Y.; Egi, M. Org. Lett. 2010, 12, 4900.

(6) Xu, W.; Zhou, Y.; Wang, R.; Wu, G.; Chen, P. Org. Biomol. Chem. 2012, 10, 367.

(7) Ikemoto, H.; Sasaki, M.; Takeda, K. Eur. J. Org. Chem. 2010, 34, 6643.

(8) Arai, N.; Azuma, K.; Nii, N.; Ohkuma, T. Angew. Chem., Int. Ed. 2008, 47, 7457.

(9) Müller, C. A.; Pfaltz, A. Angew. Chem., Int. Ed. 2008, 47, 3363.

(10) Aloise, A. D.; Layton, M. E.; Shair, M. D. J. Am. Chem. Soc. 2000, 122, 12610.

(11) Wang, M.; Xie, M.-H.; Wu, C.-D.; Wang, Y.-G. Chem.Commun. 2009, 2396.

(12) Lafrance, M.; Roggen, M.; Carreira, E. M. Angew. Chem., Int. Ed. 2012, 51, 3470.

(13) Kanbayashi, N.; Onitsuka, K. Angew. Chem., Int. Ed. 2011, 50, 5197.

(14) Marion, N., Gealageas, R.; S. Nolan, P. Org. Lett. 2007, 9, 2653.

(15) Lyothier, I.; Defieber, C., Carreira, E. M. Angew. Chem., Int. Ed., 2006, 45, 6204.

(16) Zhu, A.; Li, L.; Wang, J.; Zhuo, K. Green Chem. 2011, 13, 1244.

(17) Wu, W.; Rao, W.; Er, Y. Q.; Loh, J. K.; Poh, C. Y.; Chan, P. W. H Tetrahedron Lett. 2008, 49, 2620.

(18) Zhuang, M.; Du, H. Org. Biomol. Chem. 2014, 12, 4590.

(19) Manzini, S.; Poater, A.; Nelson, D. J.; Cavallo, L.; Nolan, S. P. Chem. Sci. 2014,5,180.

(20) Ding, B.; Zhang, Z.; Liu, Y.; Sugiya, M.; Imamoto, T.; Zhang, W. Org. Lett. 2013, 15, 3690.

(21) Hay, M. B.; Wolfe, J. P. Tetrahedron Lett. 2006, 47, 2793.

(22) Gooßen, L. J.; Ghosh, K. Eur. J. Org. Chem. 2002, 19, 3254.

(23) Shang, J.-Y.; Li, F.; Bai, X.-F.; Jiang, J.-X.; Yang, K.-F.; Lai, G.-Q.; Xu, L.-W. Eur. J. Org. Chem. 2012, 2809.

(24) Huang, H.; Ji, X.; Wu, W.; Huang, L.; Jiang, H.; J. Org. Chem. 2013, 78, 3774.

(25) Endo, K.; Hamada, D.; Yakeishi, S.; Shibata, T. Angew. Chem., Int. Ed. 2013, 52, 606.

(26) Chan, L. K. M.; Poole, D. L.; Shen, D.; Healy, M. P.; Donohoe, T. J.; Angew. Chem., Int. Ed. 2014, 53,761 .

(27) Katritzky, A. R.; Zhang, G.; Jiang, J. J. Org. Chem., 1995, 60, 7605. 
(28) Mori, Y.; Seki, M. Adv. Synth. Catal. 2007, 349, 2027.

(29) Katritzky, A. R.; Le, K. N. B.; Khelashvili, L.; Mohapatra, P. P. J. Org. Chem. 2006, 71, 9861.

(30) Ueno, S.; Shimizu, R.; Kuwano, R. Angew. Chem., Int. Ed. 2009, 48, 4543.

(31) Uyanik, M.; Suzuki, D.; Yasui, T.; Ishihara, K. Angew. Chem., Int. Ed. 2011, 50, 5331.

(32) Endo, K.; Hamada, D.; Yakeishi, S.; Shibata, T. Angew. Chem., Int. Ed. 2013, 52, 606.

(33) Stroba, A.; Schaeffer, F.; Hindie, V.; Lopez-Garcia, L.; Adrian, I.; Fröhner, W.; Hartmann, R.W.; Biondi, R. M.; Engel, M. J. Med. Chem.2009, 52, 4683.

(34) Saito, S.; Sato, Y.; Ohwada, T.; Shudo, K. J. Am. Chem. Soc. 1994,116, 2312.

(35) Ding, B.; Zhang, Z.; Liu, Y.; Sugiya, M.; Imamoto, T.; Zhang, W. Org. Lett. 2013, 15, 3690.

(36) Kurita, T.; Aoki, F.; Mizumoto, T.; Maejima, T.; Esaki, H.; Maegawa, T.; Monguchi, Y.; Sajiki, H. Chem. Eur. J. 2008, 14, 3371. 


\section{NMR spectra}
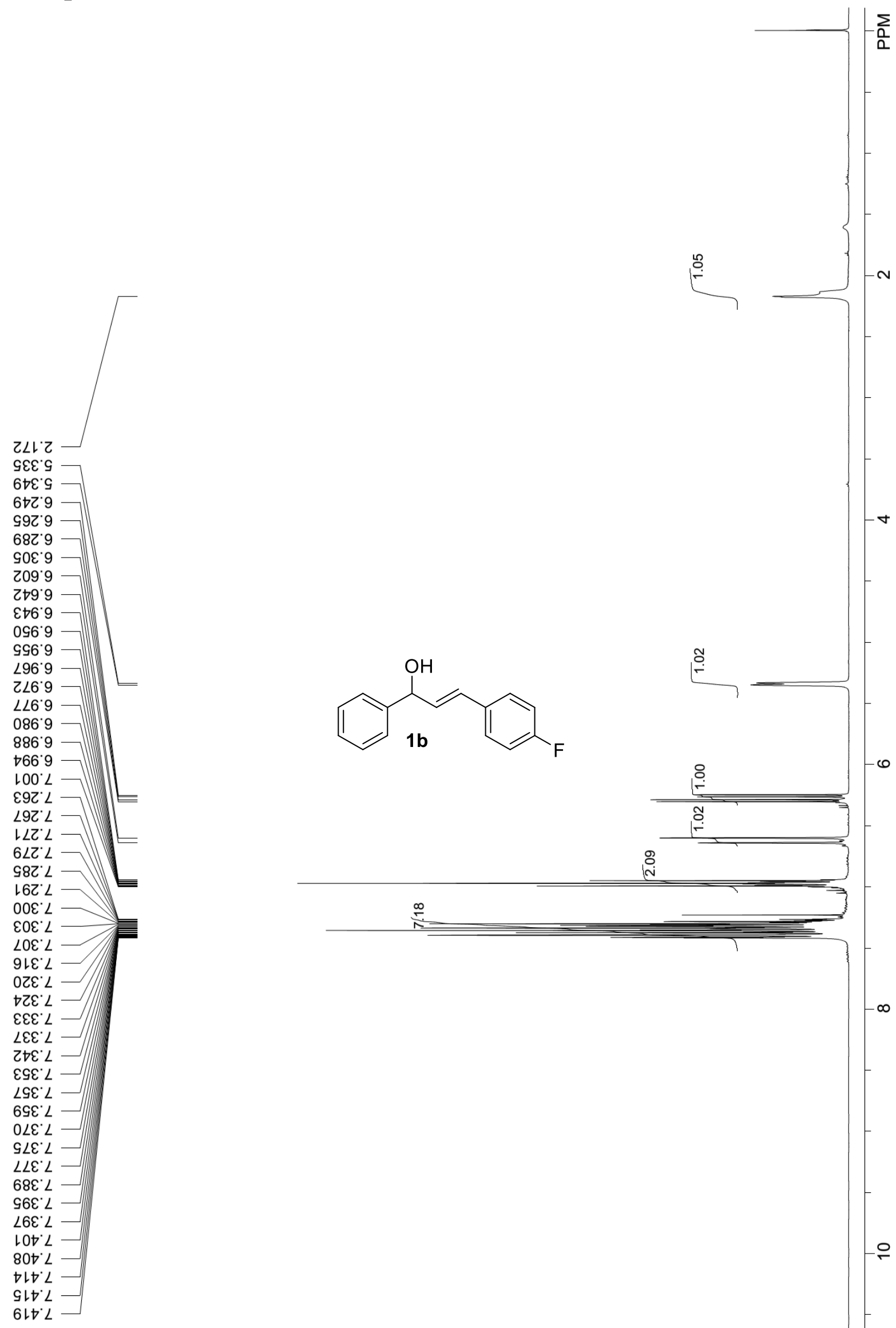

i

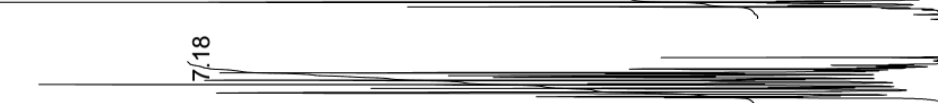

$\infty$

S-33 

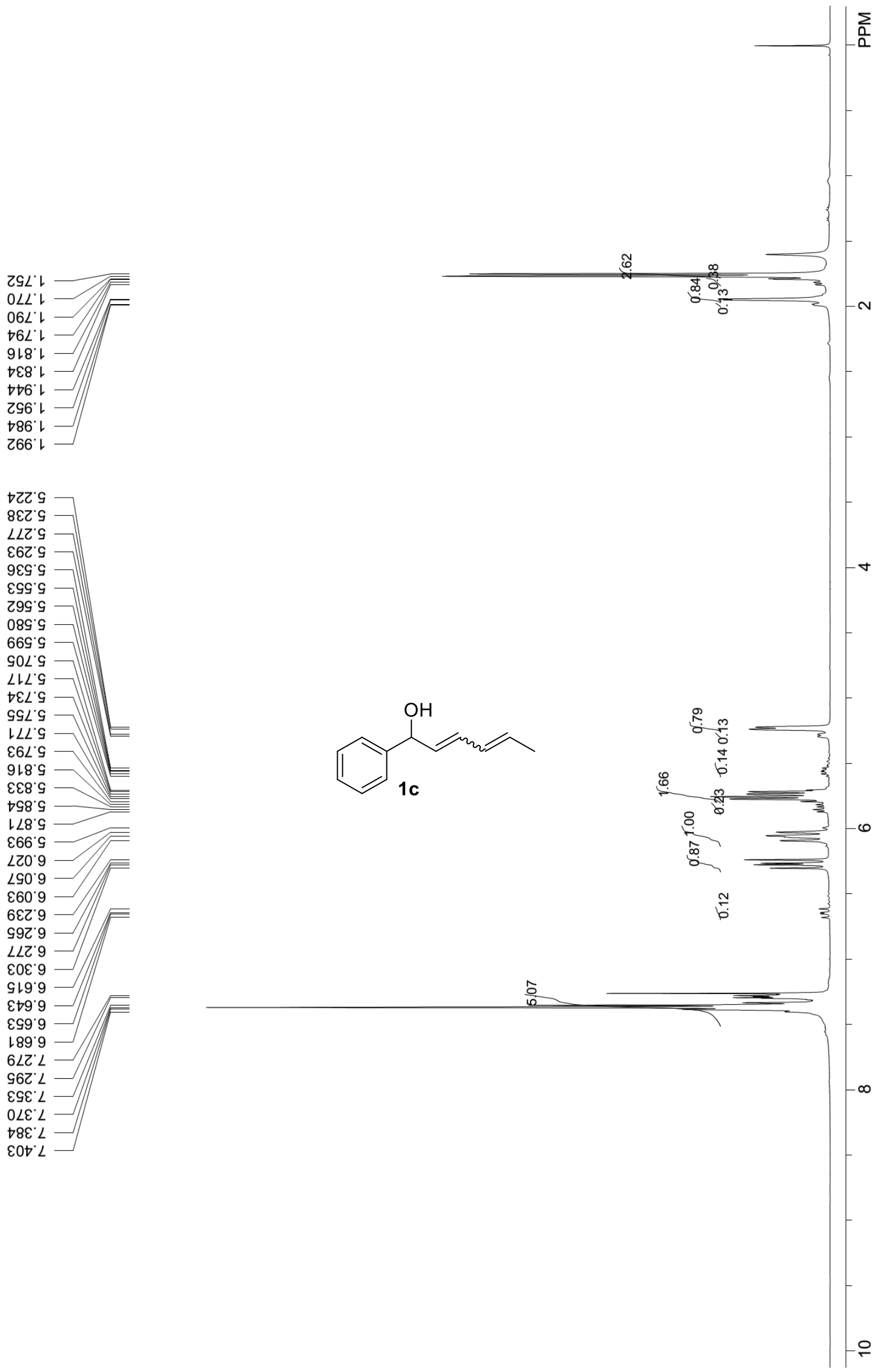

S-34 
$862 \mathrm{C}$

$91 \varepsilon^{\circ} \mathrm{s}$

ยะ๕ 9

$\nabla L Z \cdot 9$

เ6Z. 9

†ाE' 9

०ะย' 9

LZ9. 9

$099^{\circ} 9$

$\nabla \varepsilon Z^{\prime} L$

$\nabla G Z^{\circ}$

892 ' $L$

ह8乙 $L$

G8Z 2

$\angle 8 Z^{\circ} \angle$

$\varepsilon 0 \varepsilon^{\circ} L$

†ZE'

เEE'

$6 \succsim \varepsilon^{\circ} \angle$

乙९६

† $9 \varepsilon^{\circ} L$

$69 \varepsilon^{\circ} L$

$\varepsilon \angle \varepsilon^{\circ} L$ 

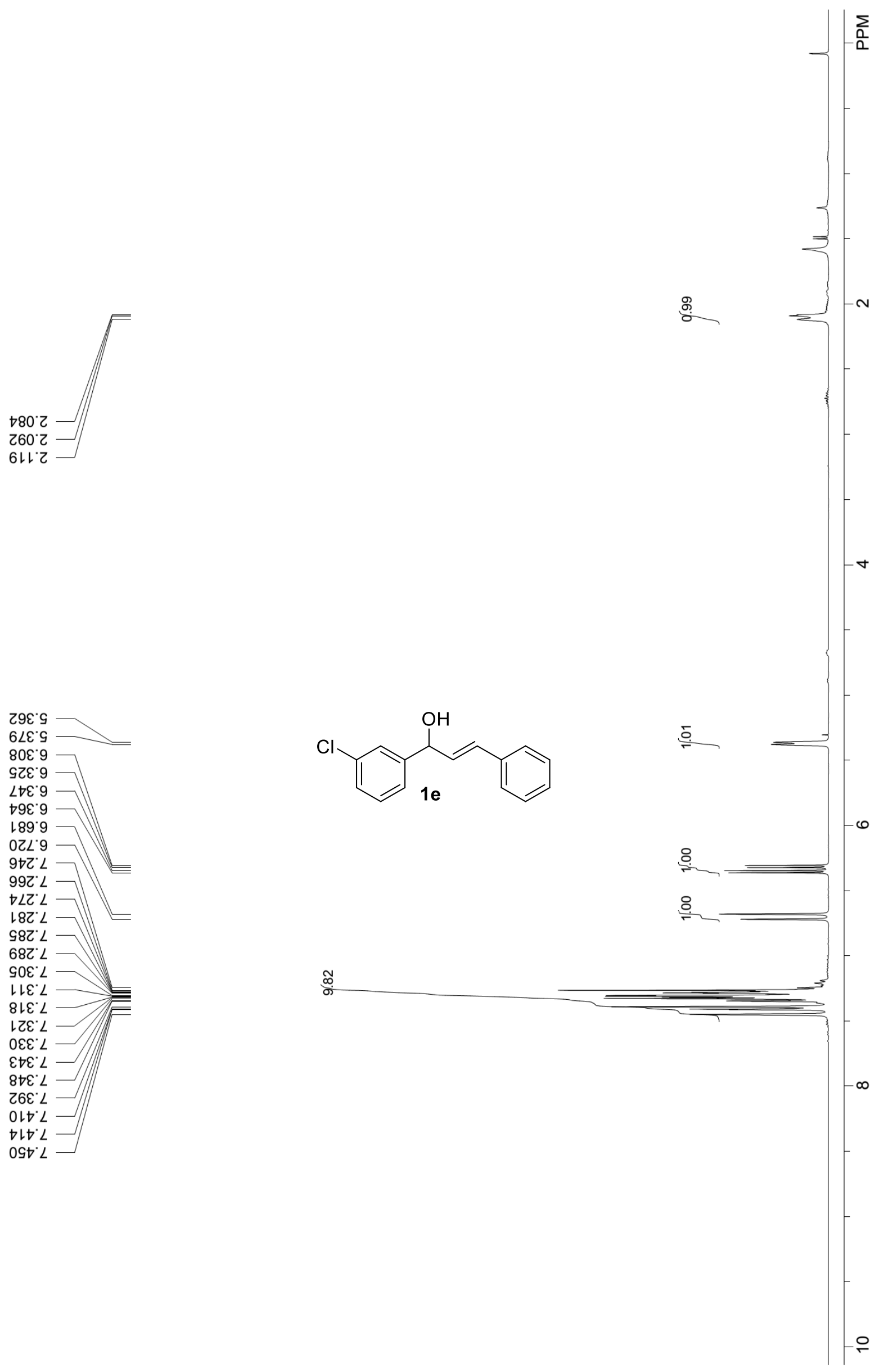

$80 \varepsilon^{\circ} 9$

与乙E'

$\downarrow 9 \varepsilon^{\circ}$

189.9

OZL'9

$9 \nabla Z$

992

$\nabla \angle Z \cdot L$

$18 Z 2$

$98 Z^{\circ} \mathrm{L}$

$68 Z^{\circ} L$

GOE' $L$

ILE. $L$

$8 เ \varepsilon^{\circ} L$

LZE' $L$

0हE' $L$

$\varepsilon \nabla \varepsilon^{\circ} L$

$8 \nabla \varepsilon^{\circ} L$

Z6E' $L$

OLt L
カレV $L$

OSt L 

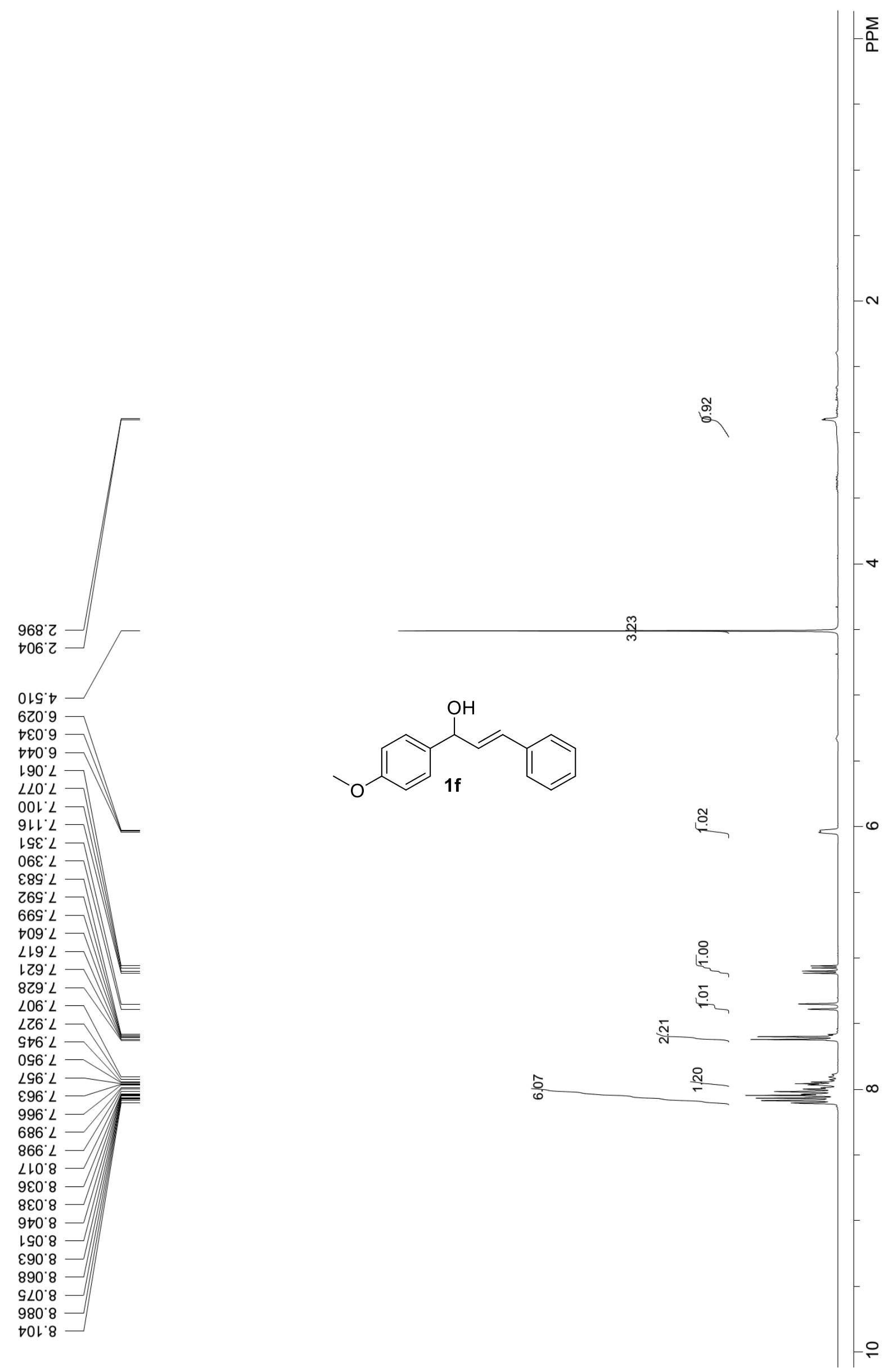

S-37 


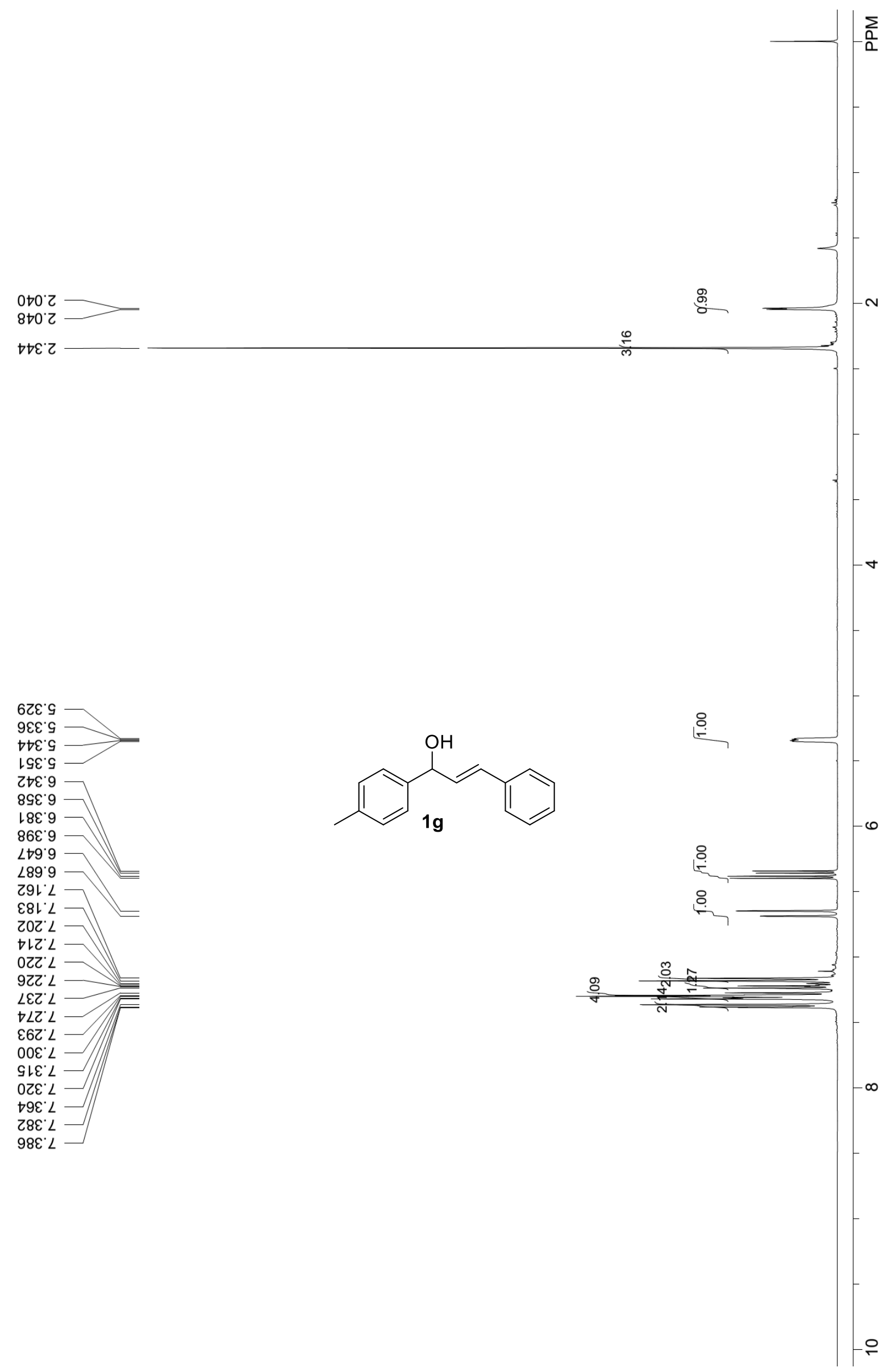

S-38 


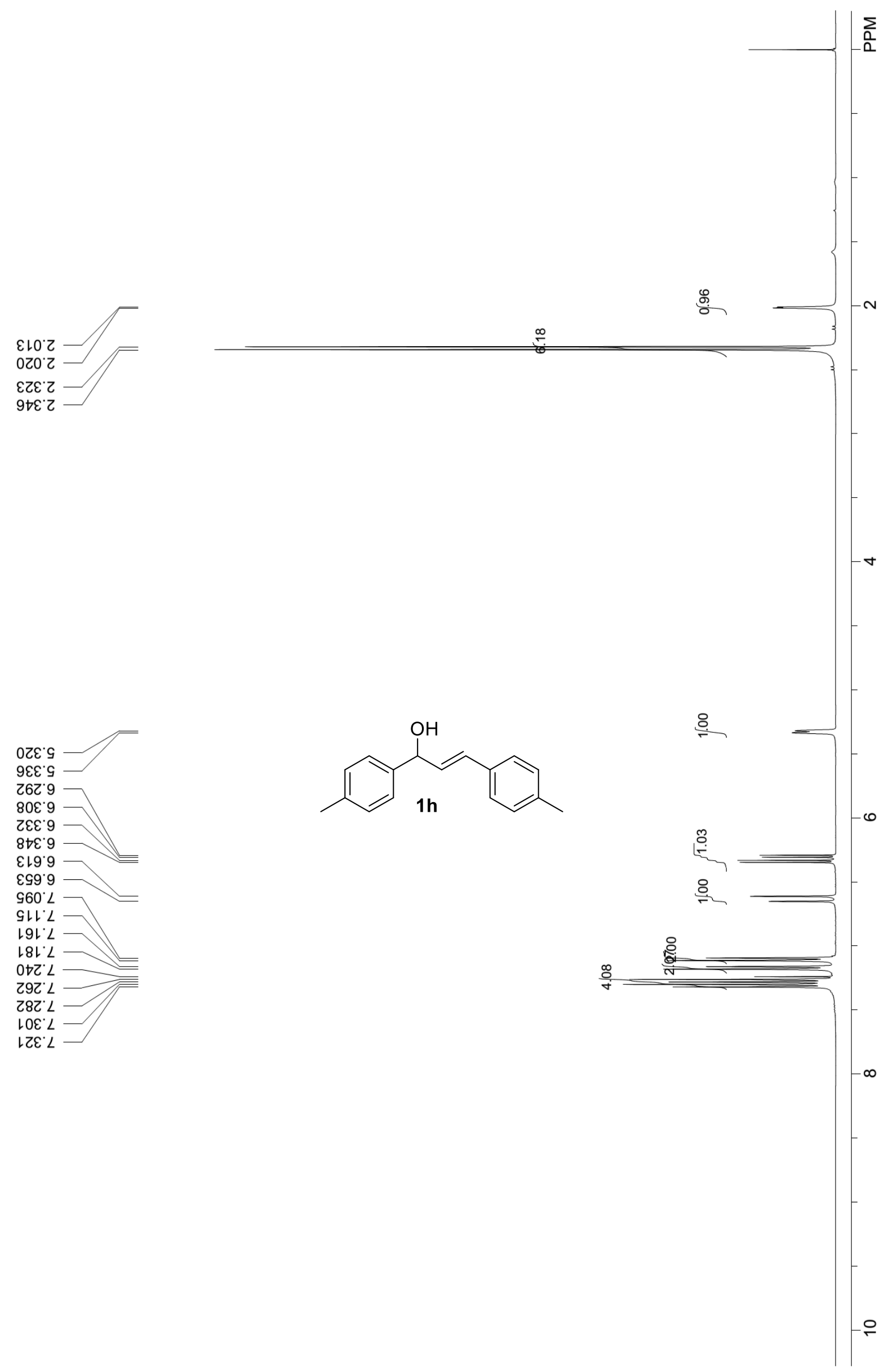

S-39 


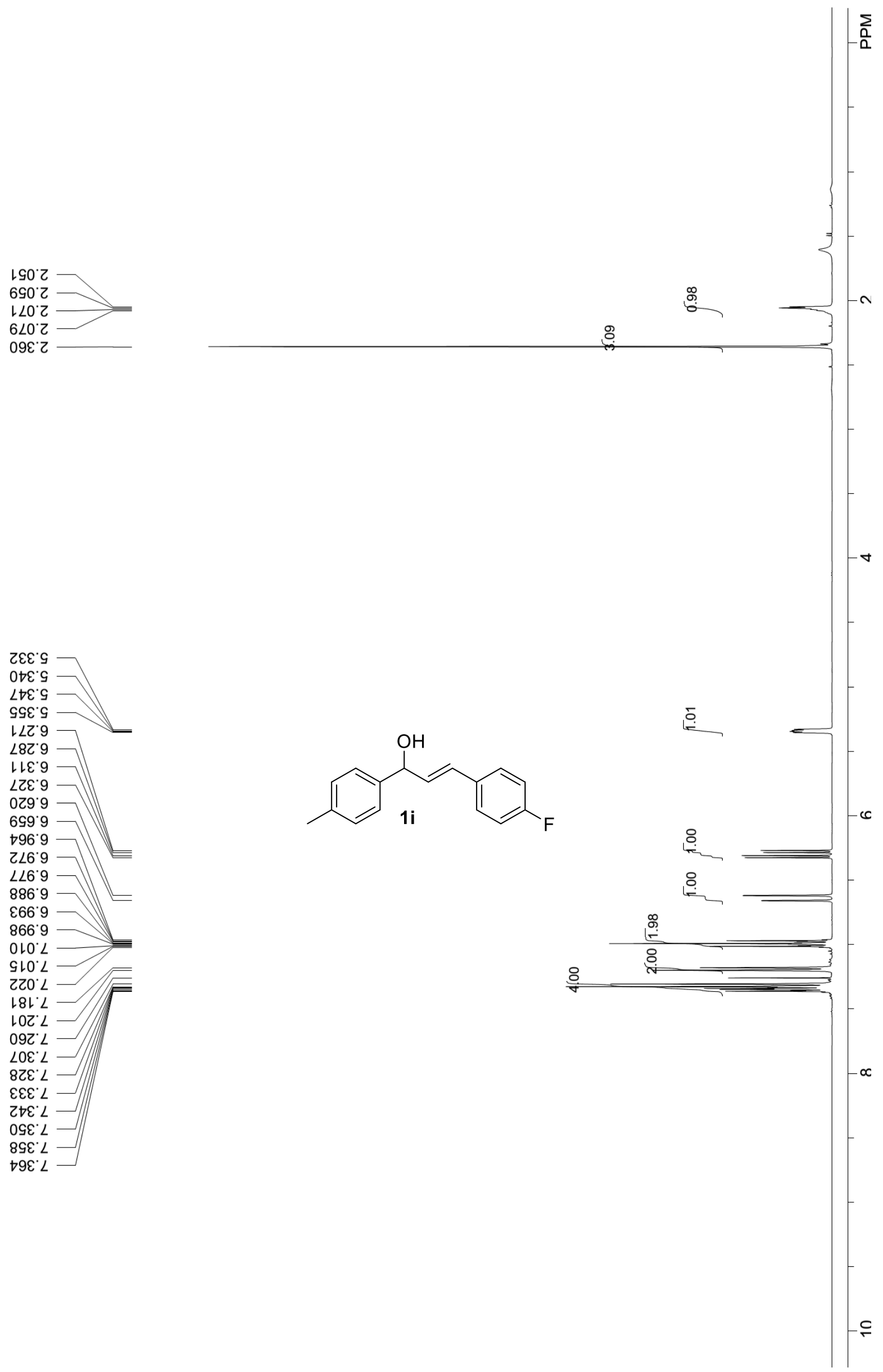



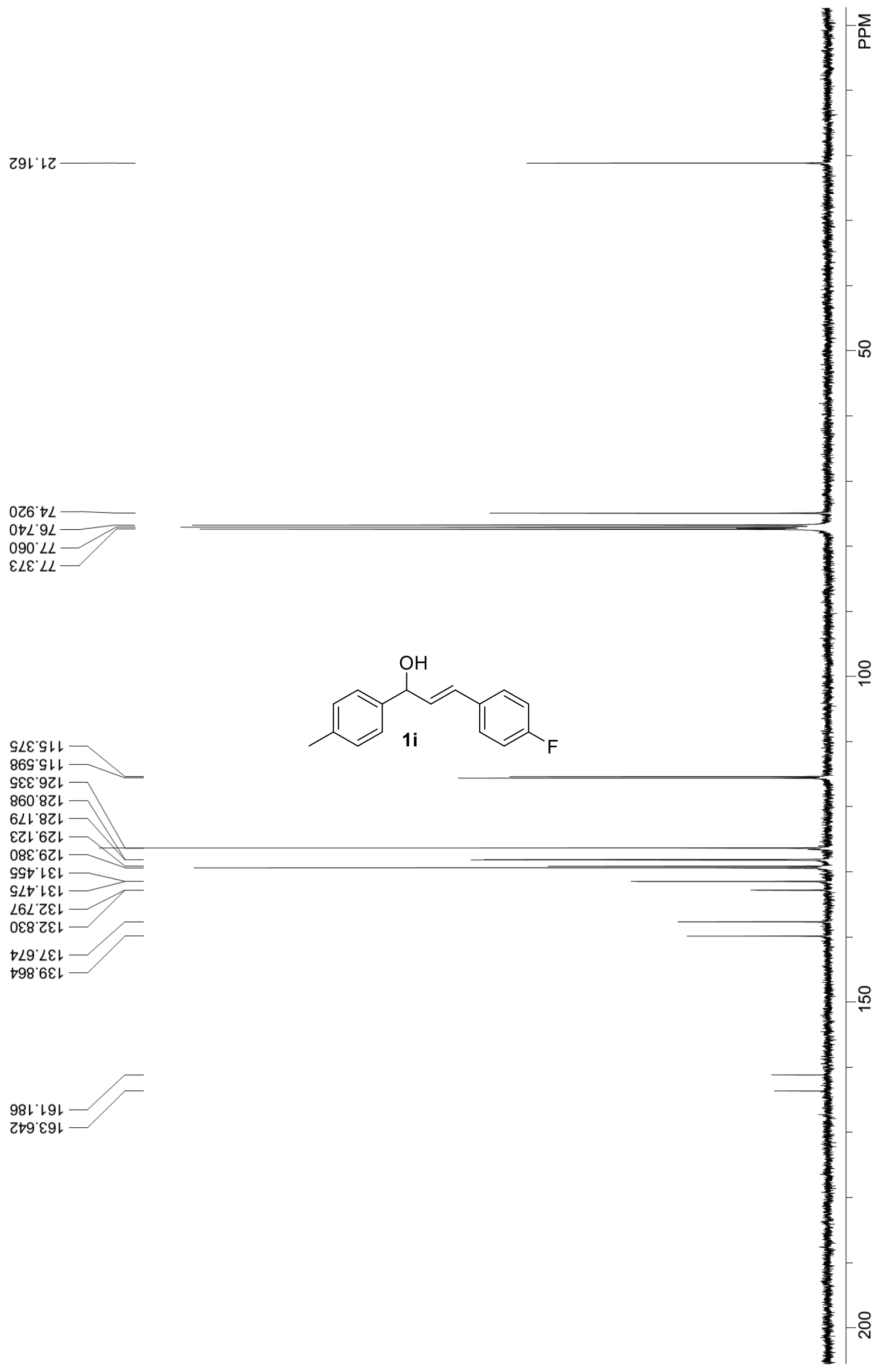


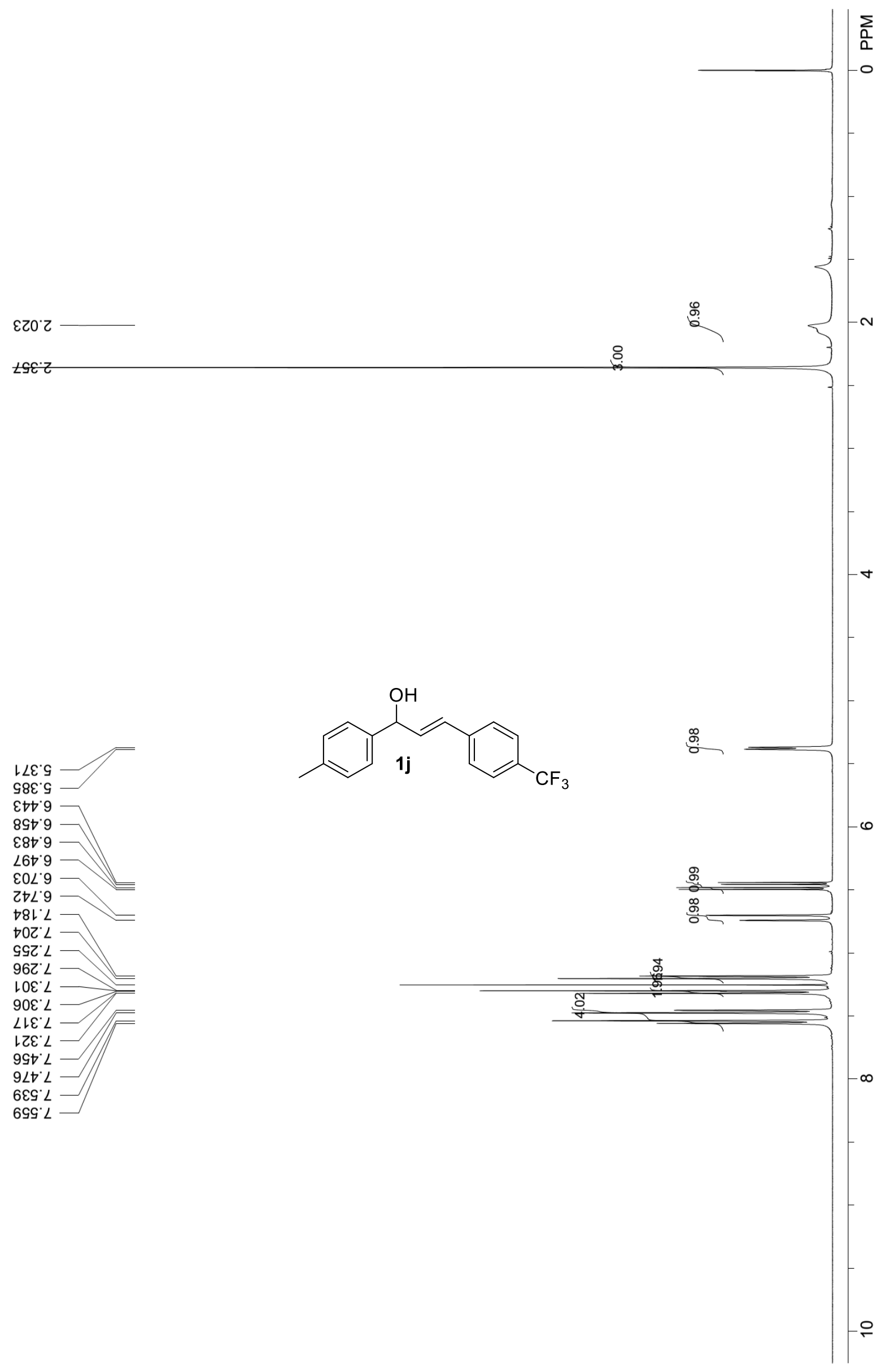



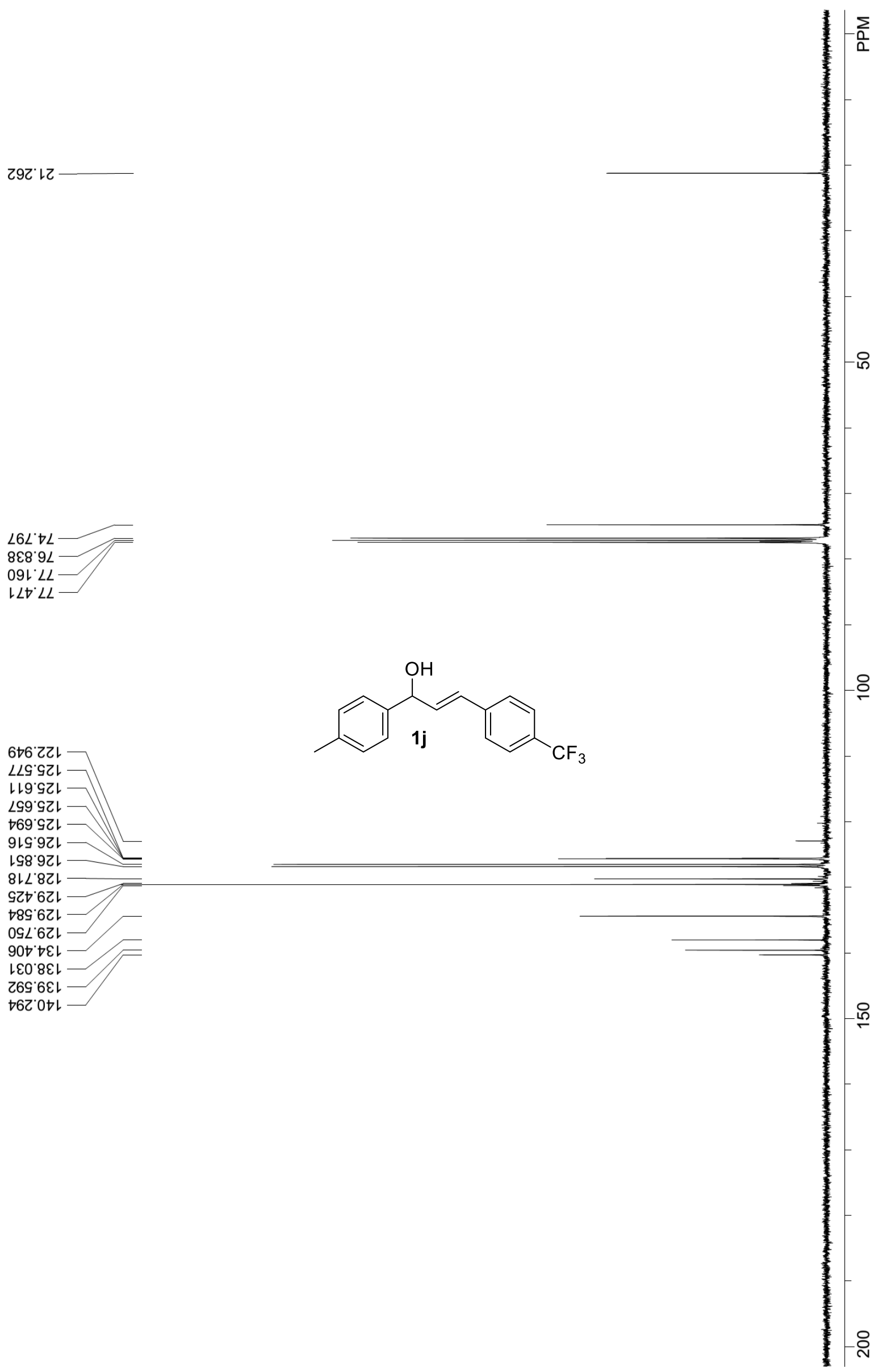

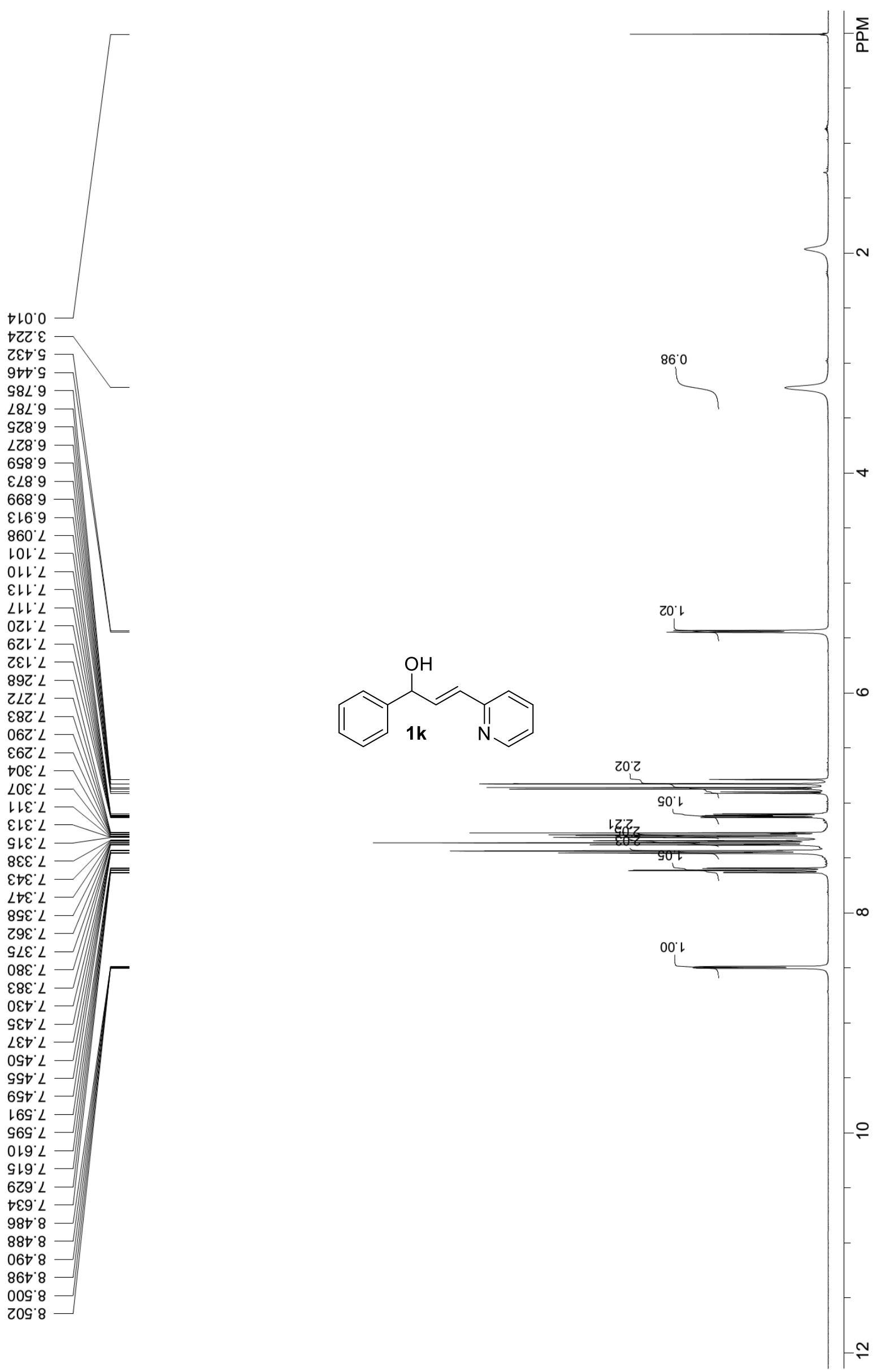


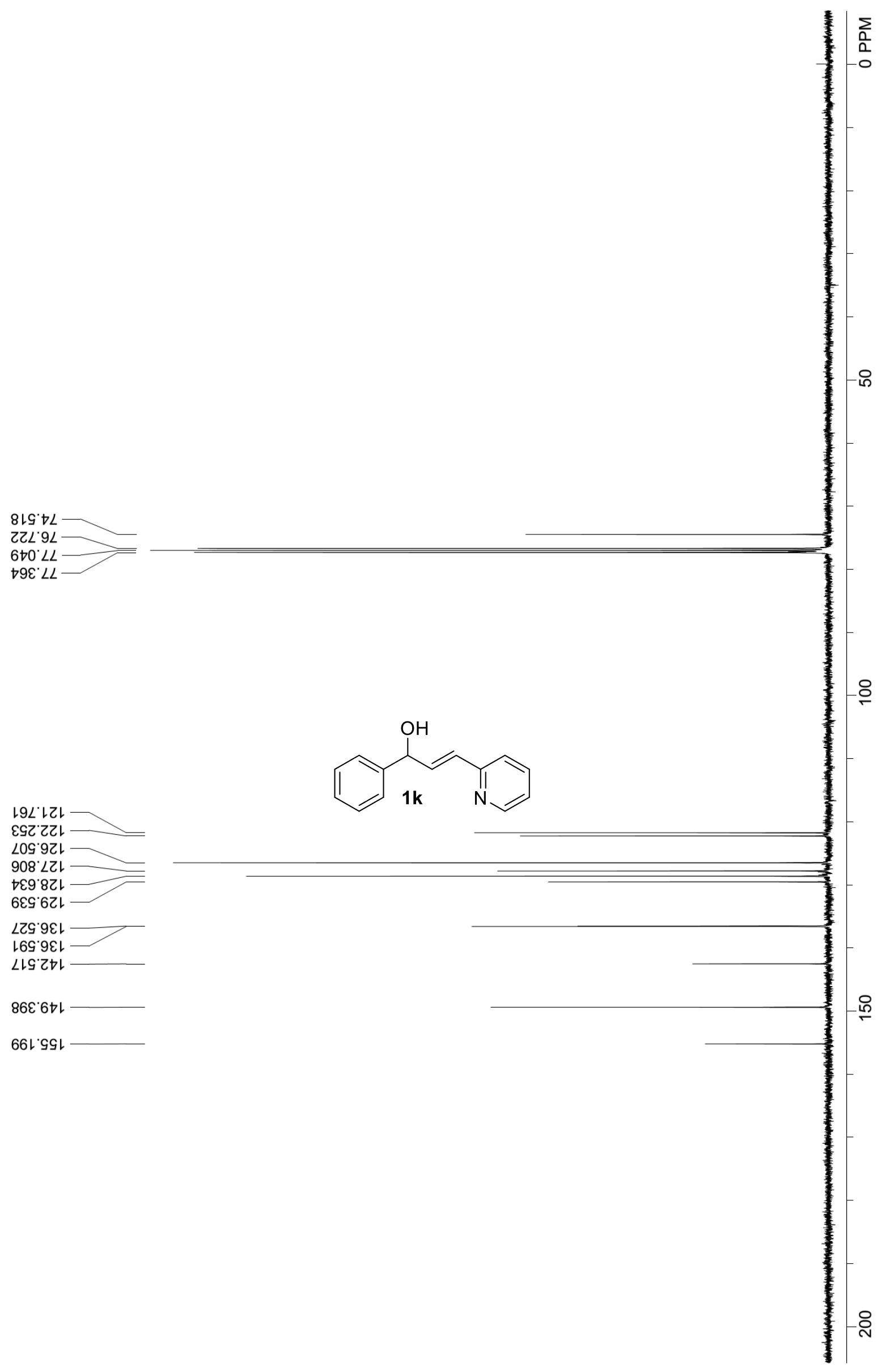




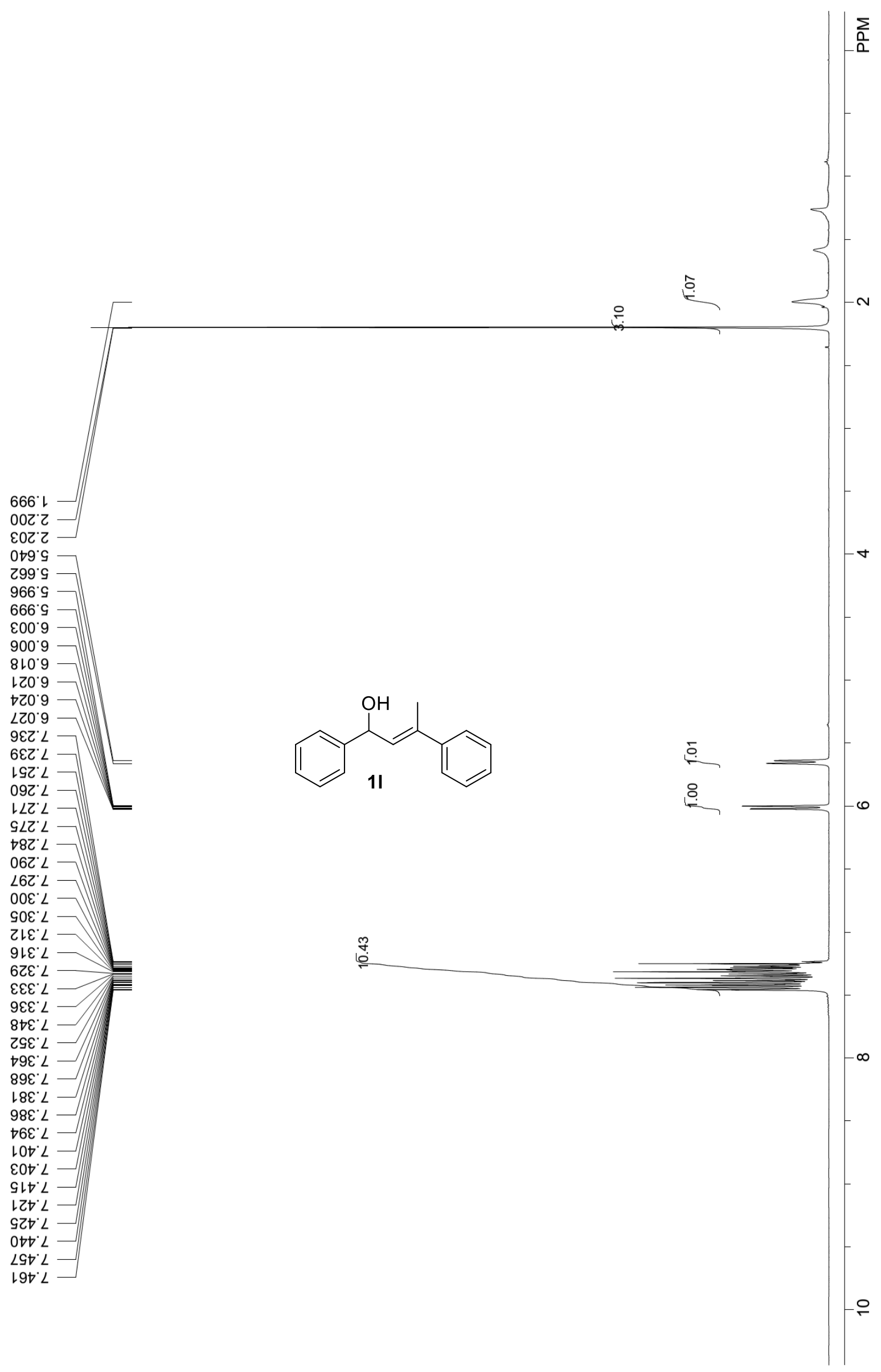



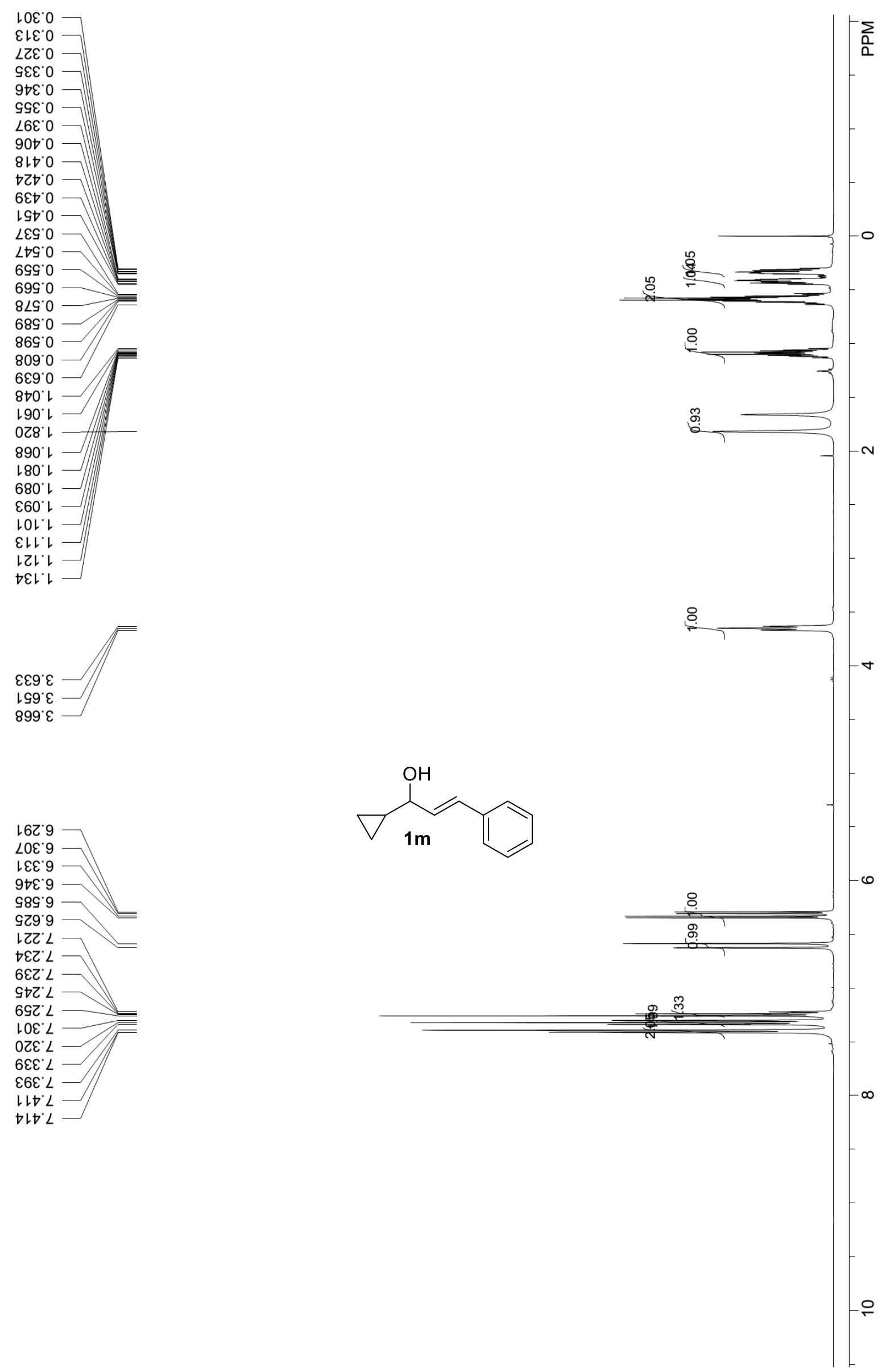

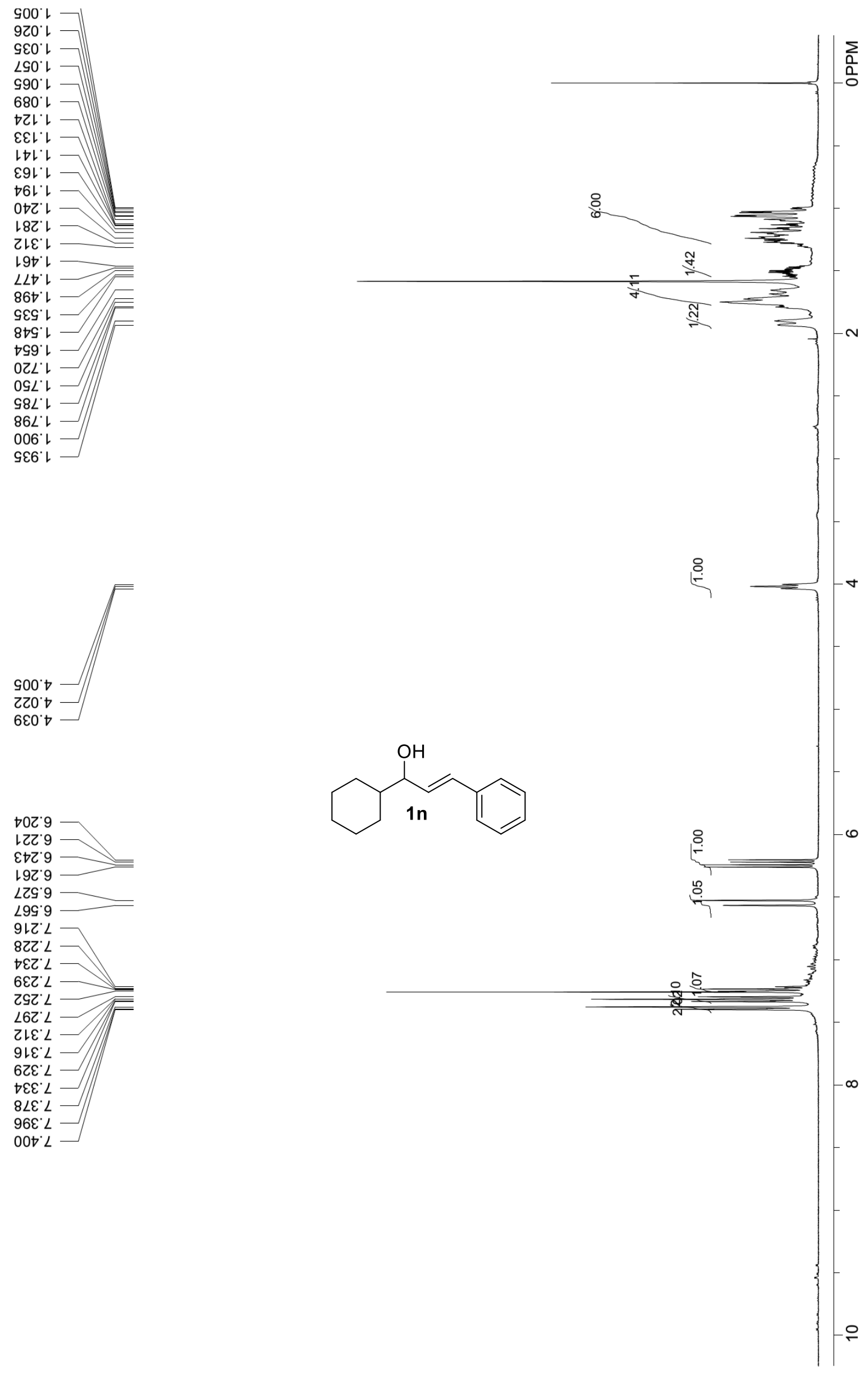


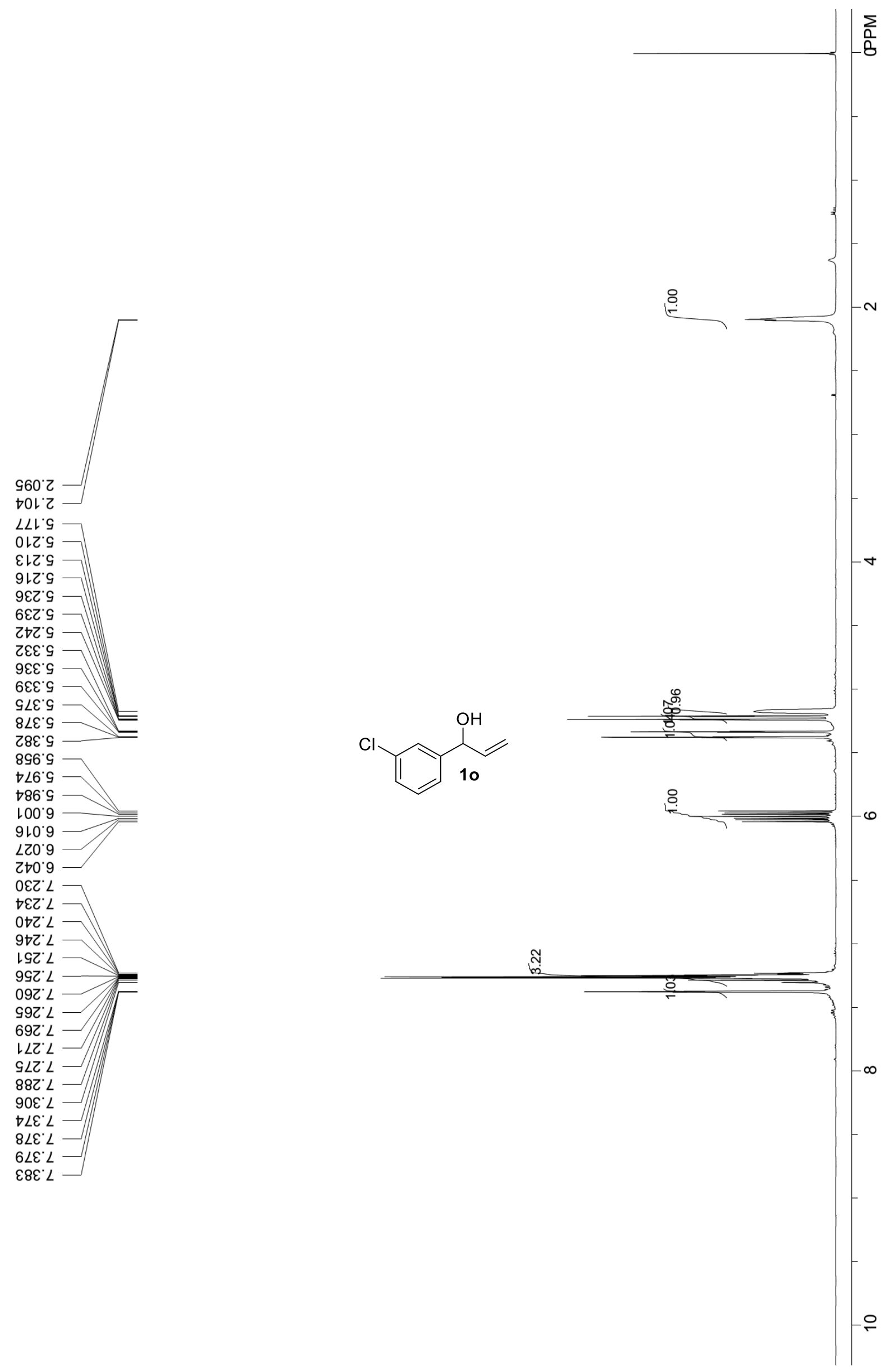


$626^{\circ} \mathrm{L}$

$8 \varepsilon 6^{\circ} \mathrm{L}$

$8 \succcurlyeq \varepsilon ' 乙$
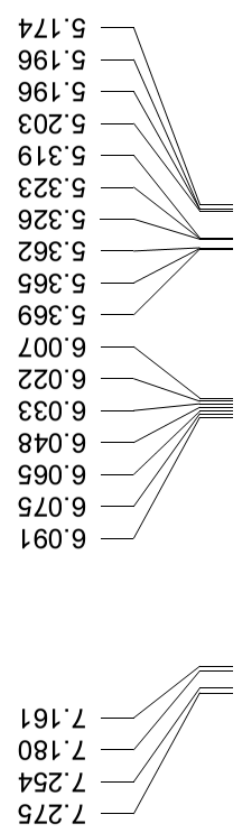

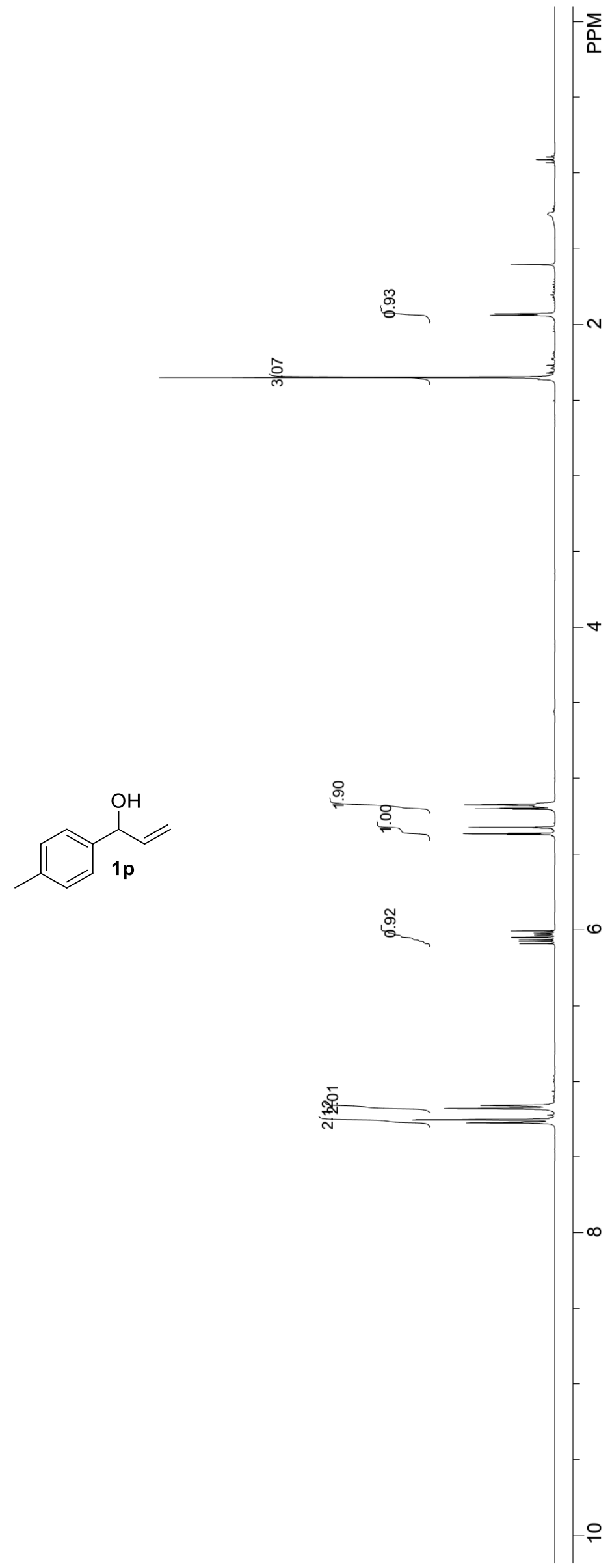

S-50 

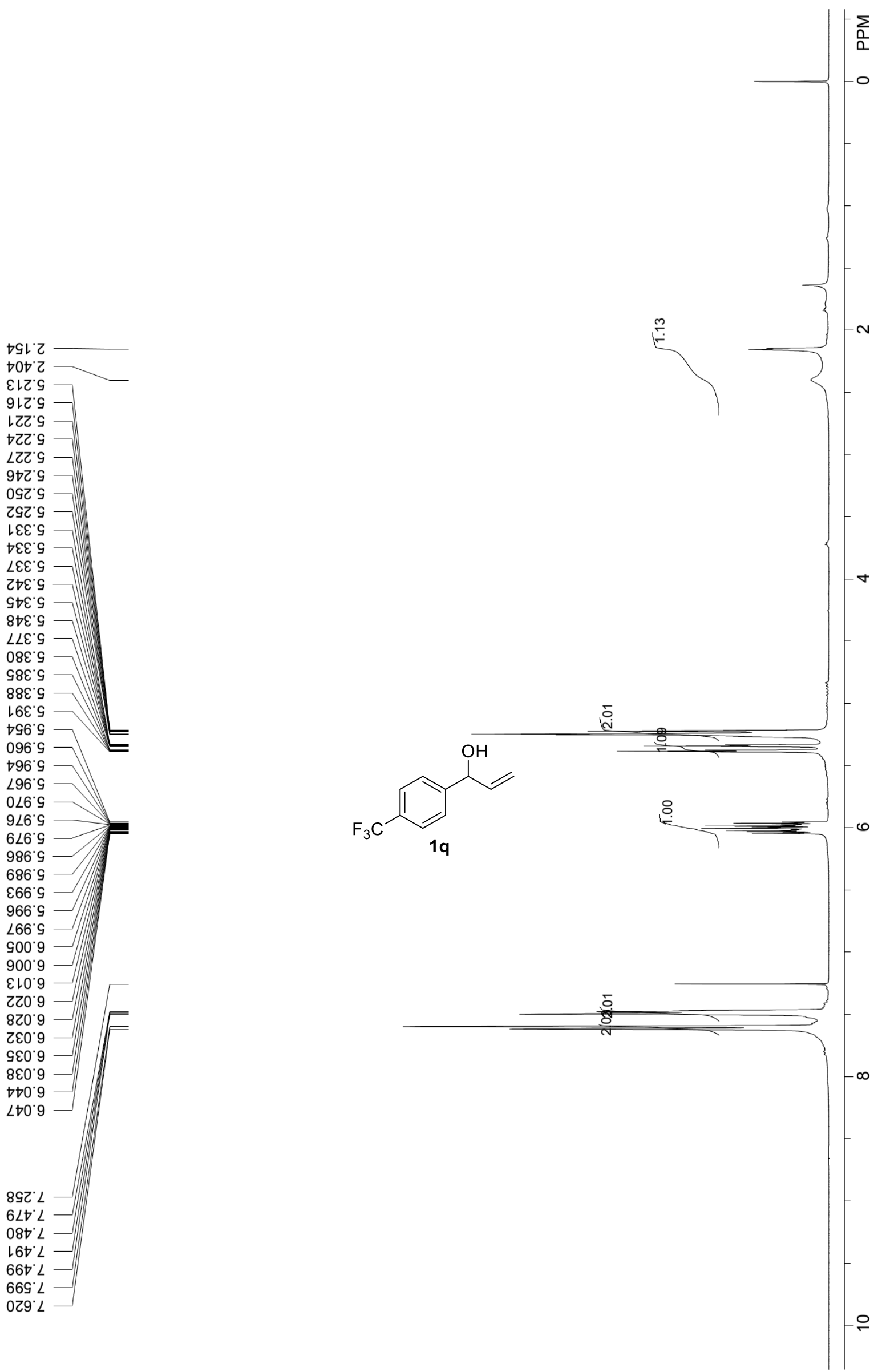

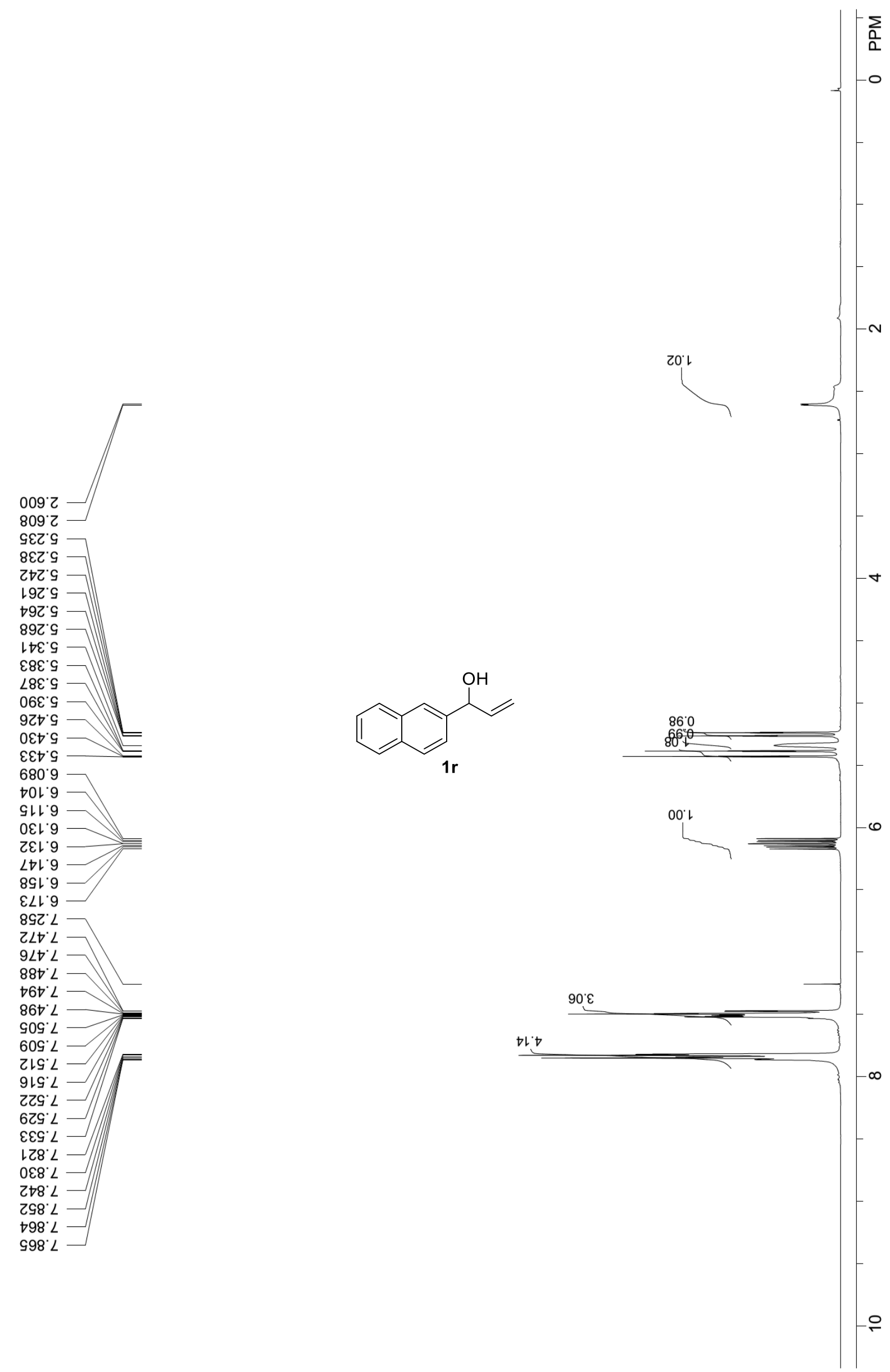

S-52 


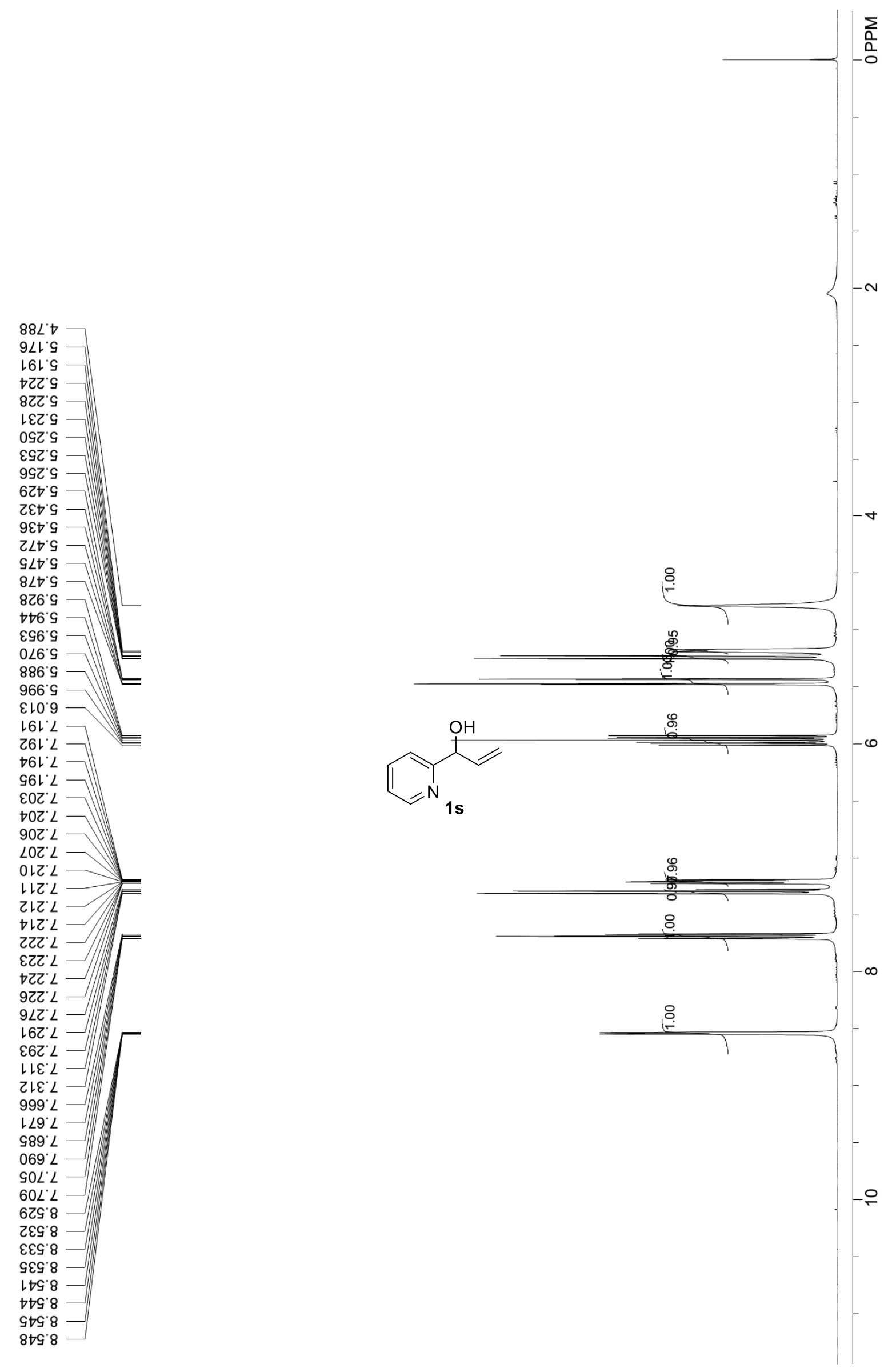

S-53 

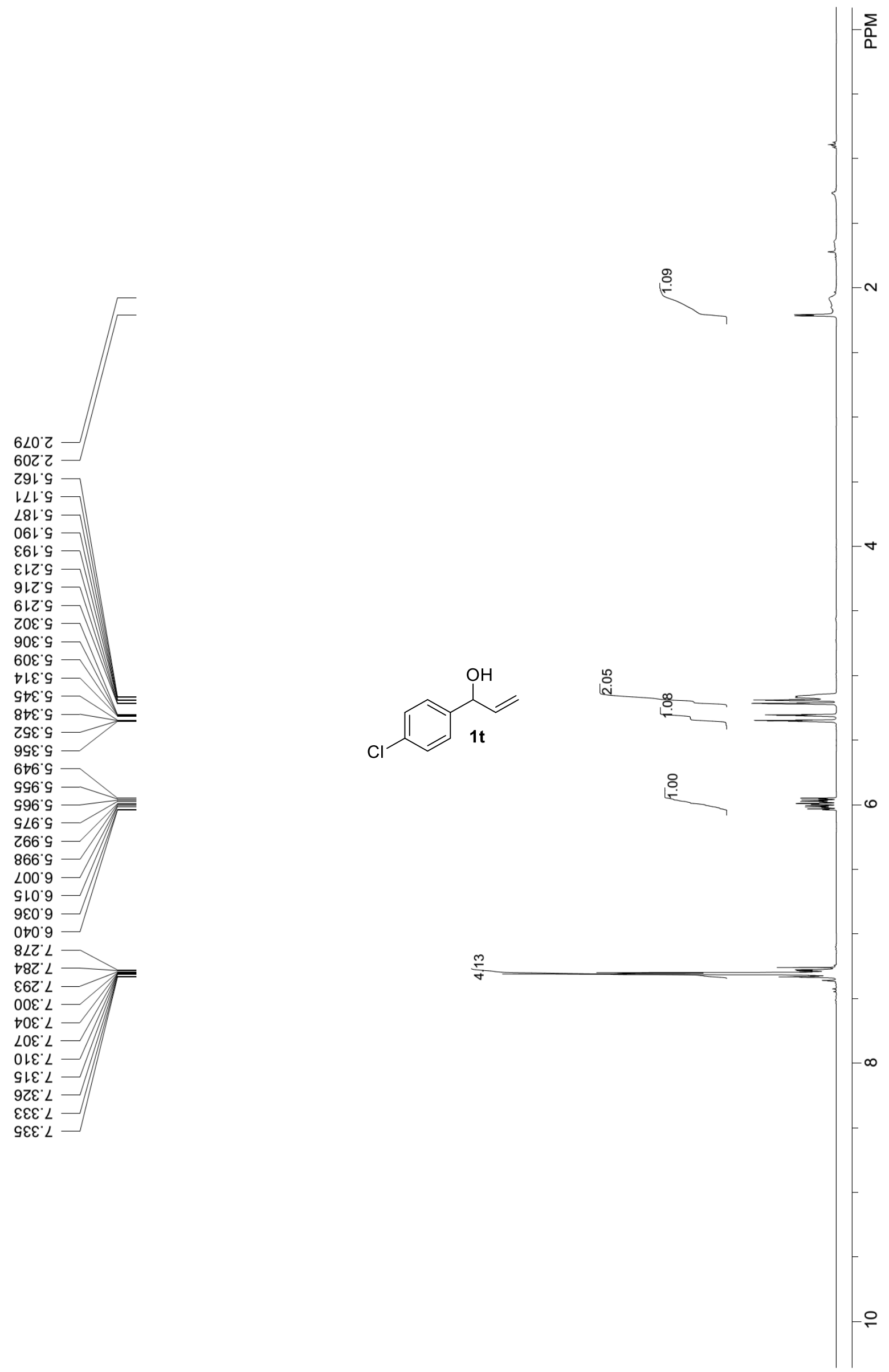

S-54 

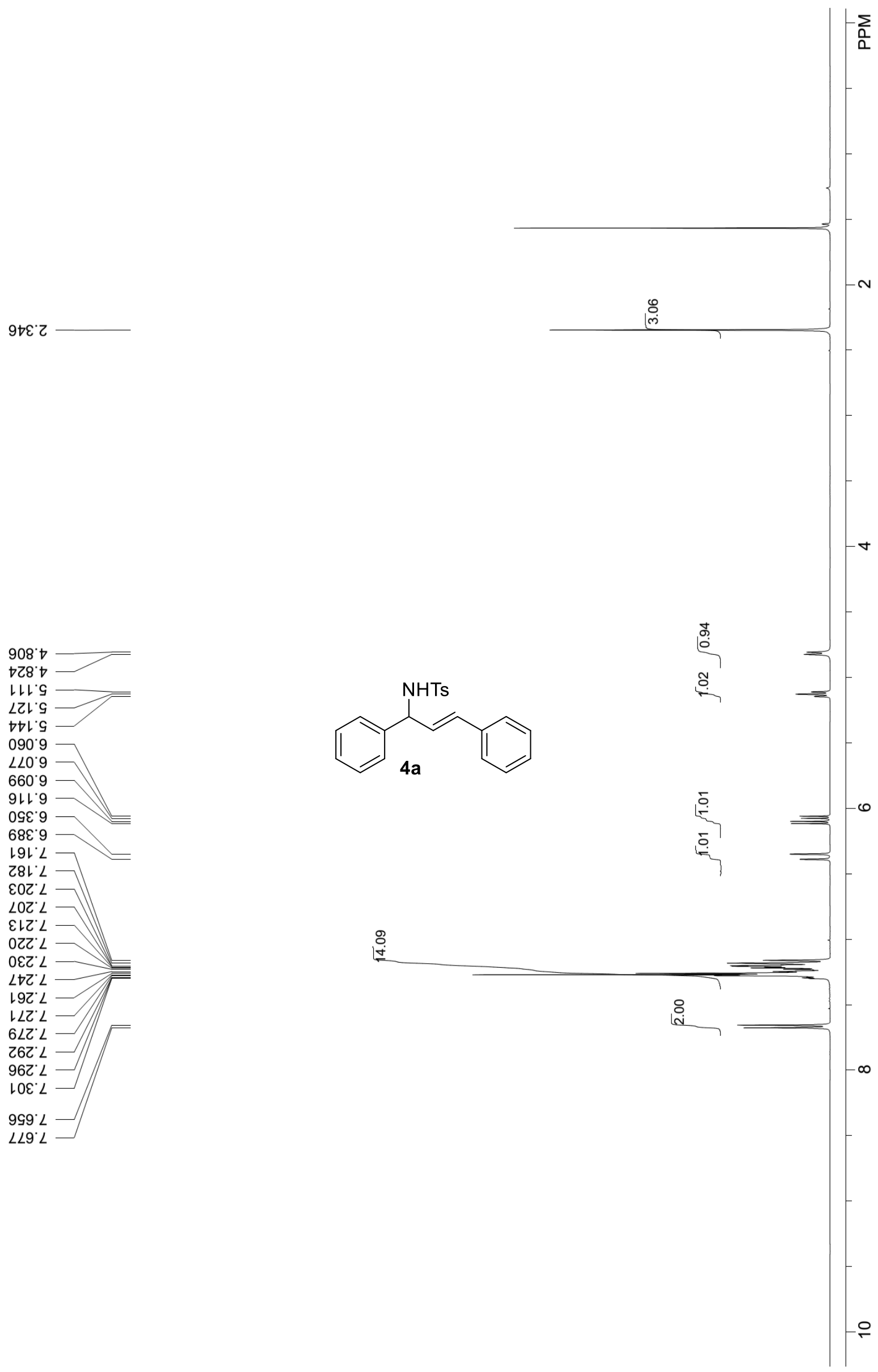


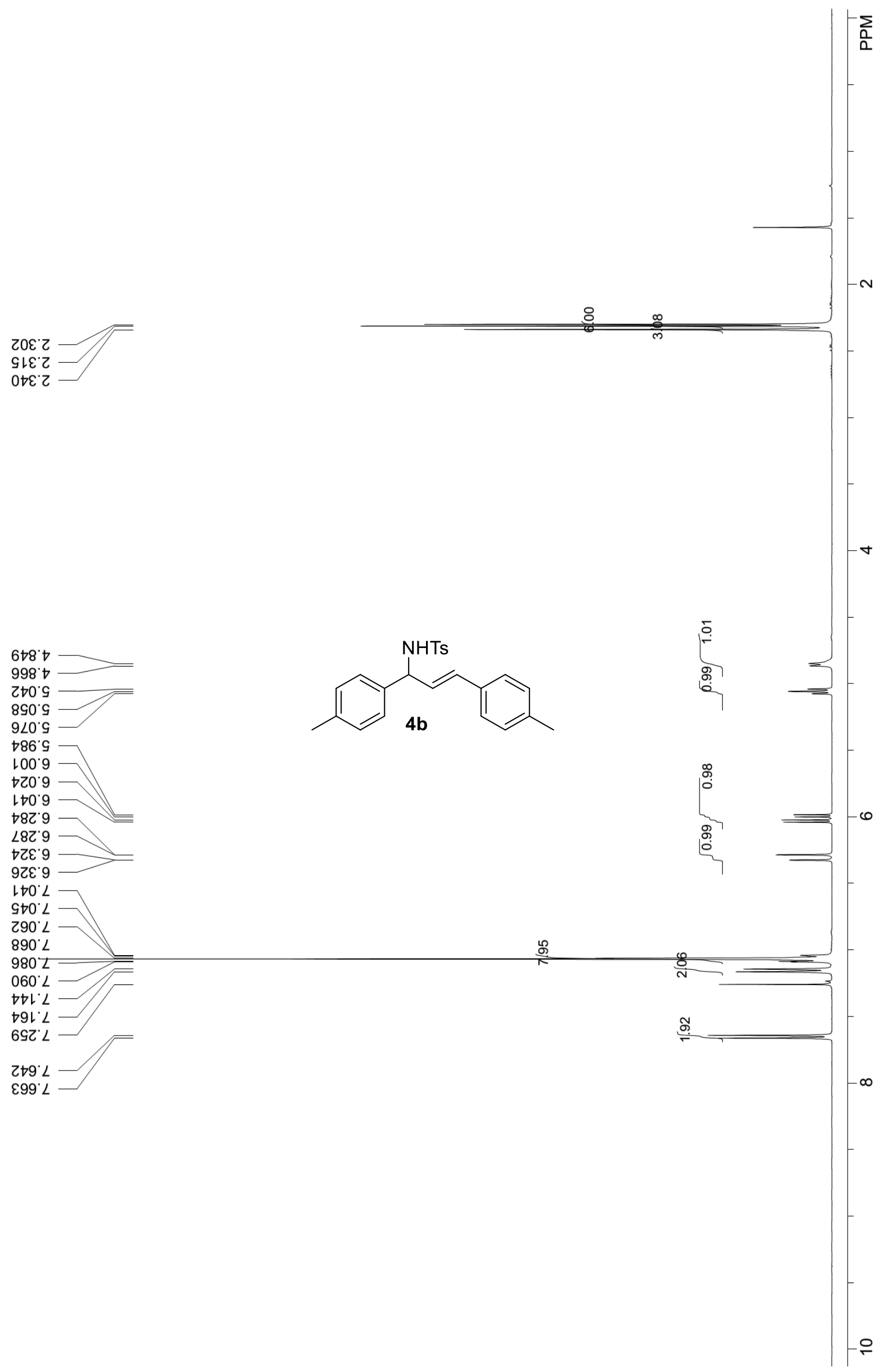



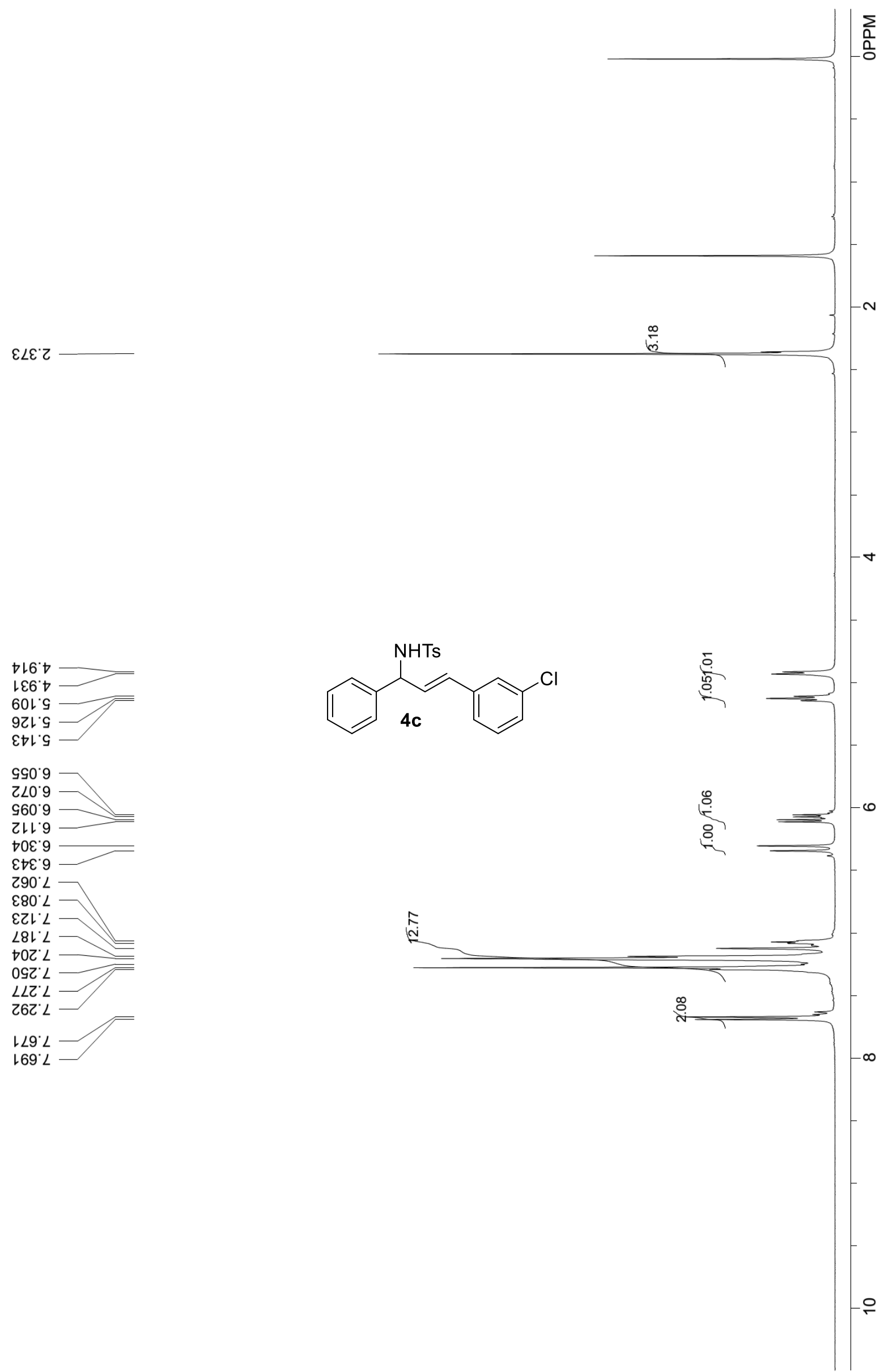

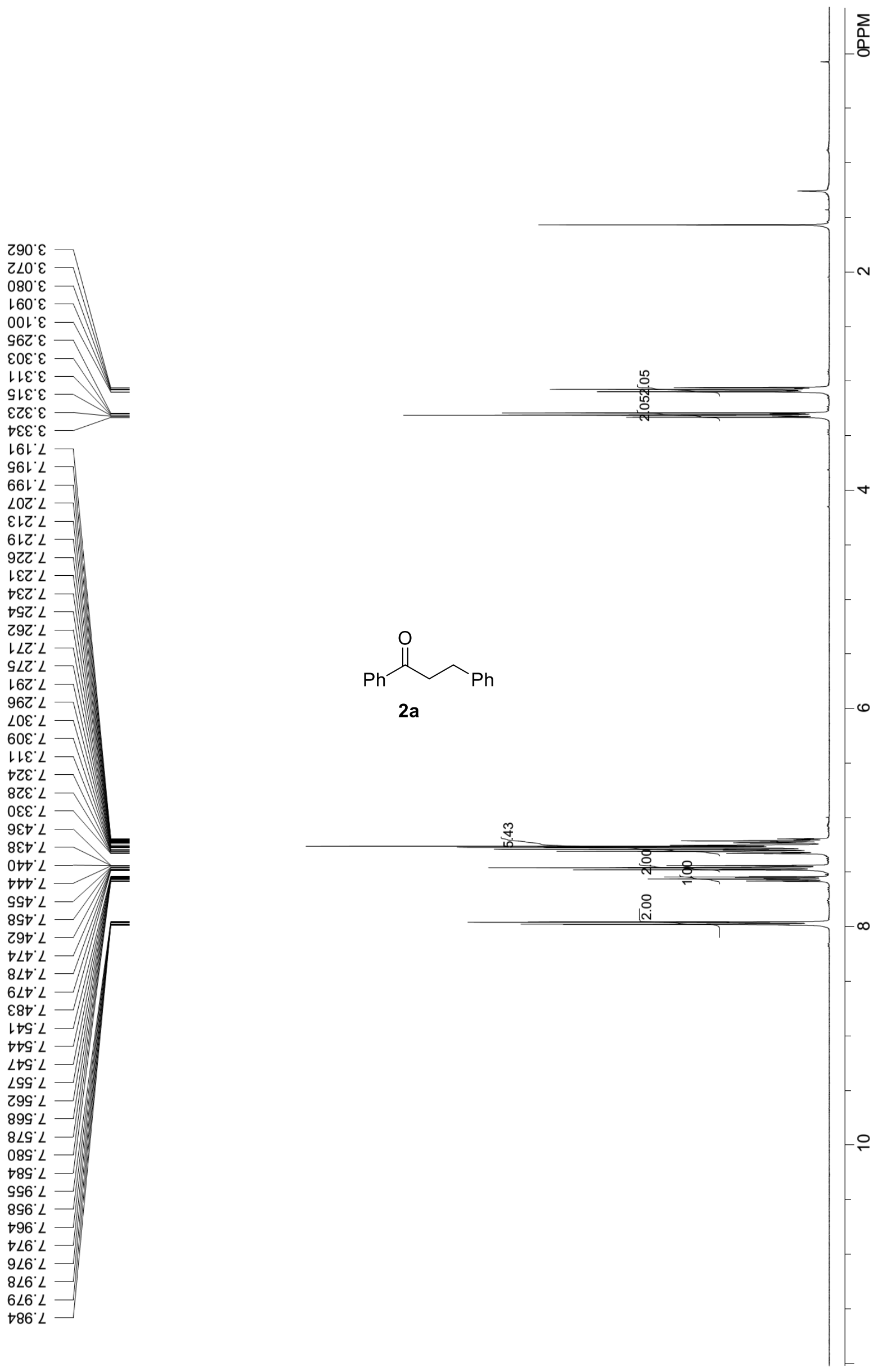


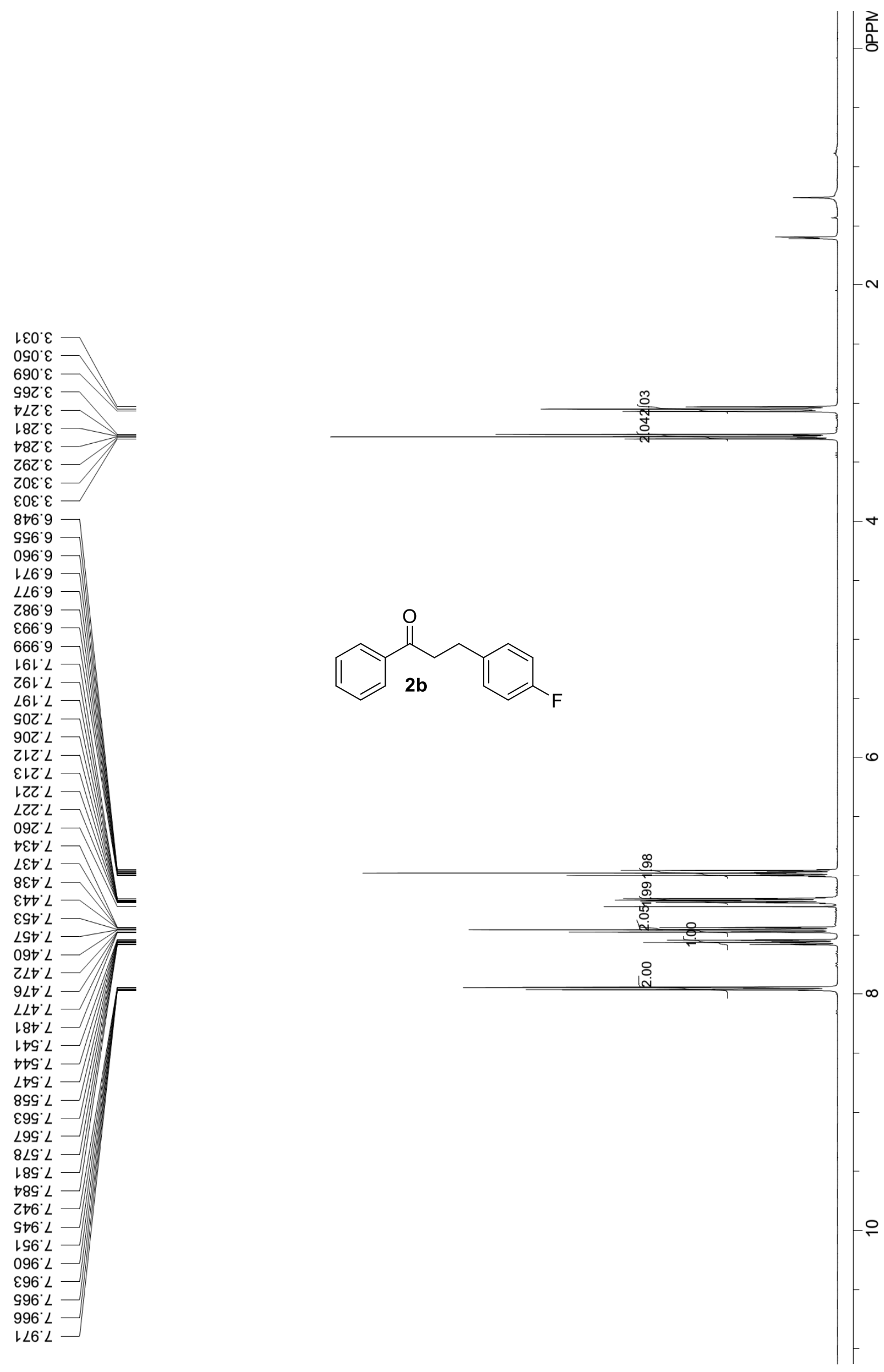



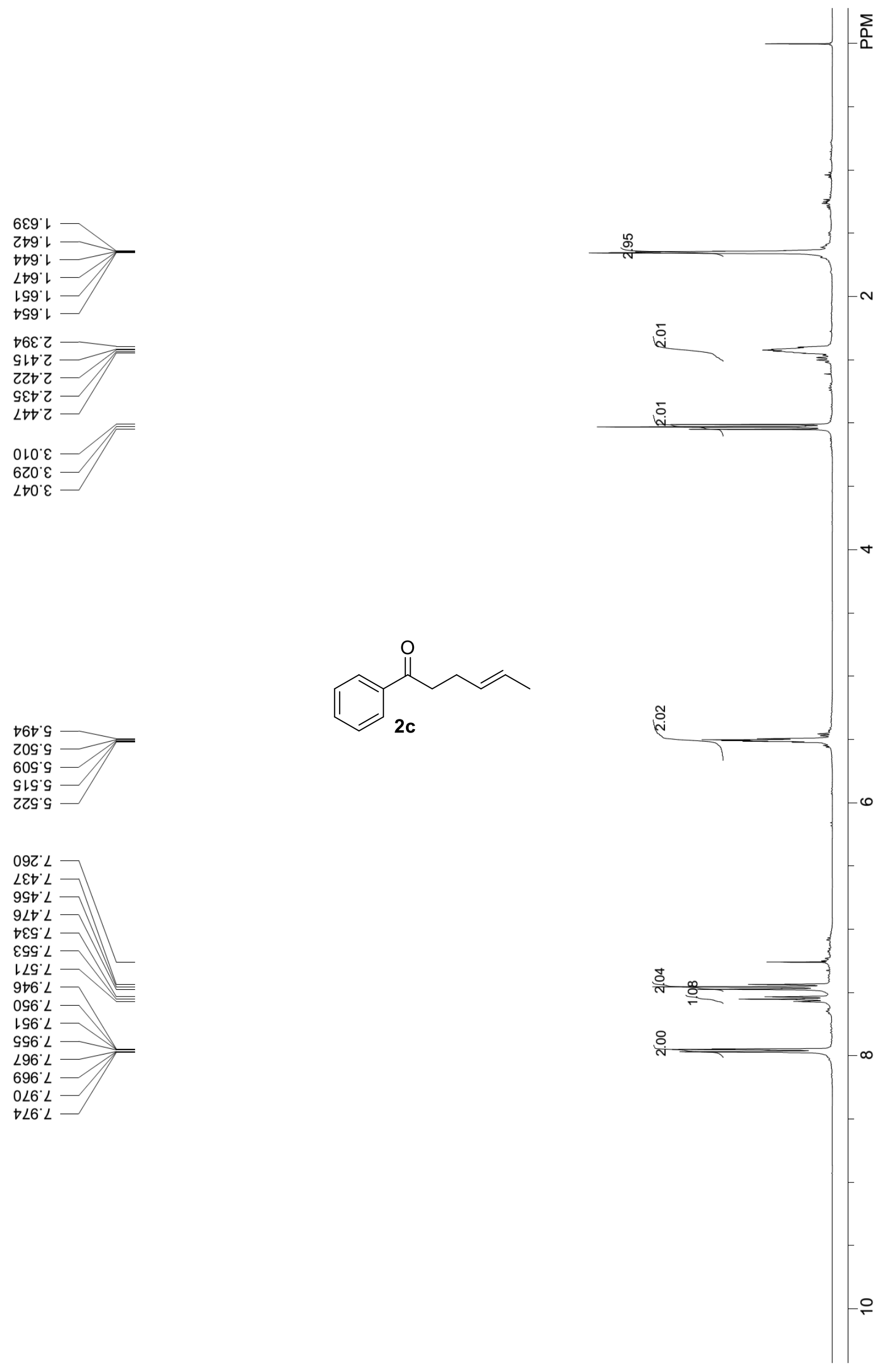

$09 Z^{\circ} \angle$

LEt $L$

$9 \angle \nabla^{\circ}$

$\downarrow \varepsilon \mathcal{G}^{\circ} \angle$

ESG $L$

ILG'

$9766^{\circ} L$

$096 . \mathrm{L}$

$196^{\circ}$

S96. $L$

$\angle 96^{\circ} \angle$

$696^{\circ}$

$0 \angle 6^{\circ} \angle$

$\nabla \angle 6^{\circ} L$
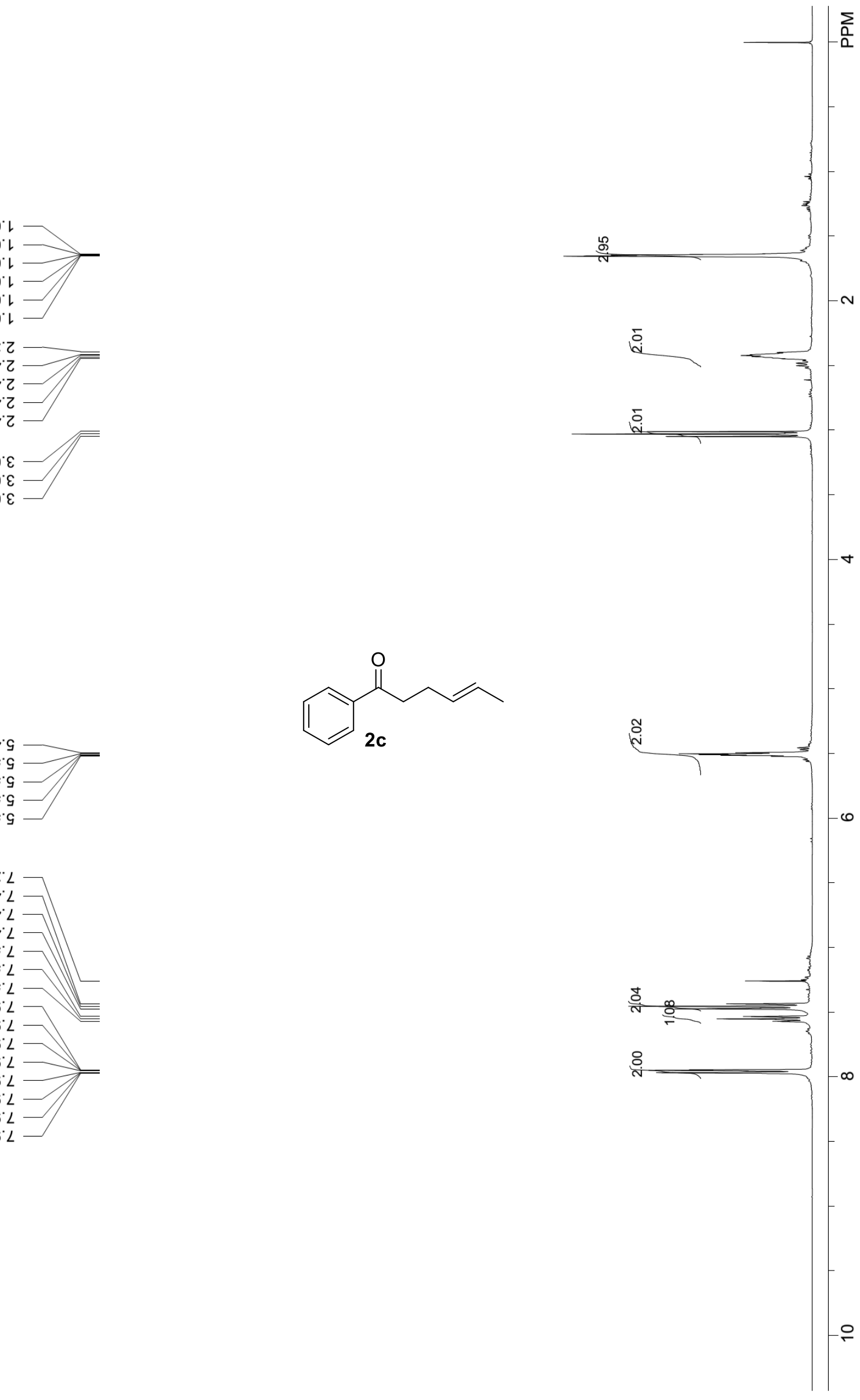

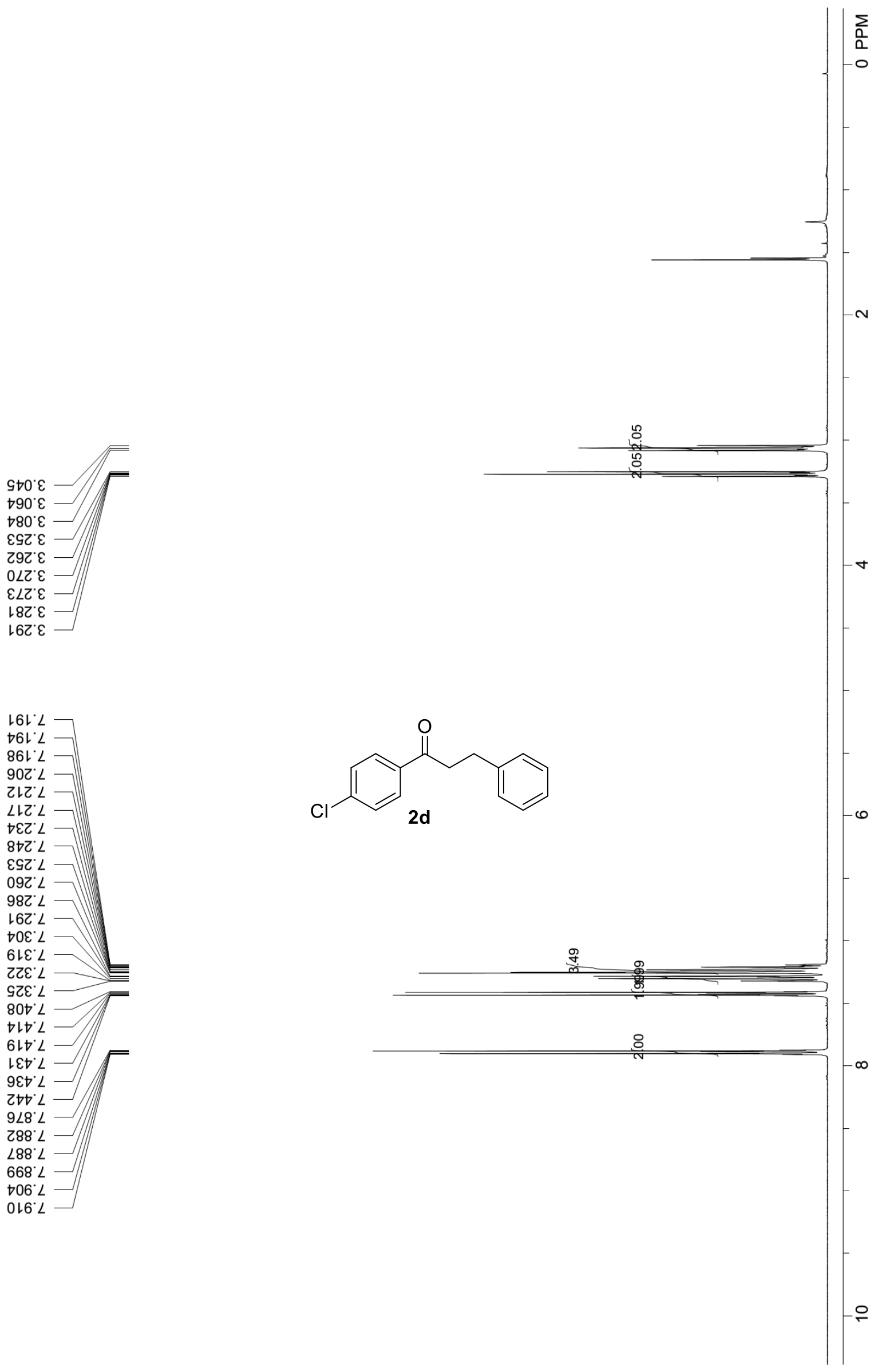


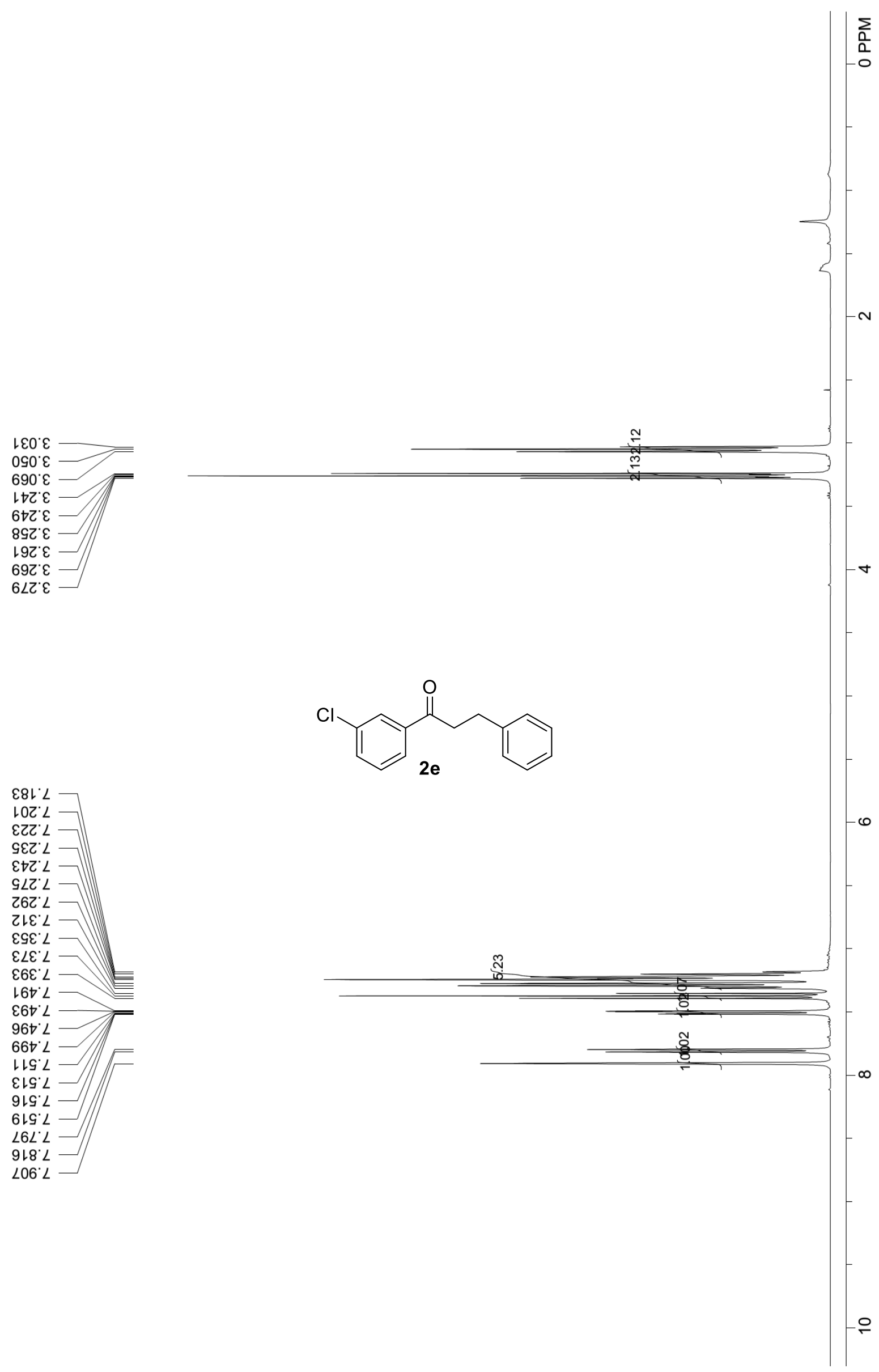



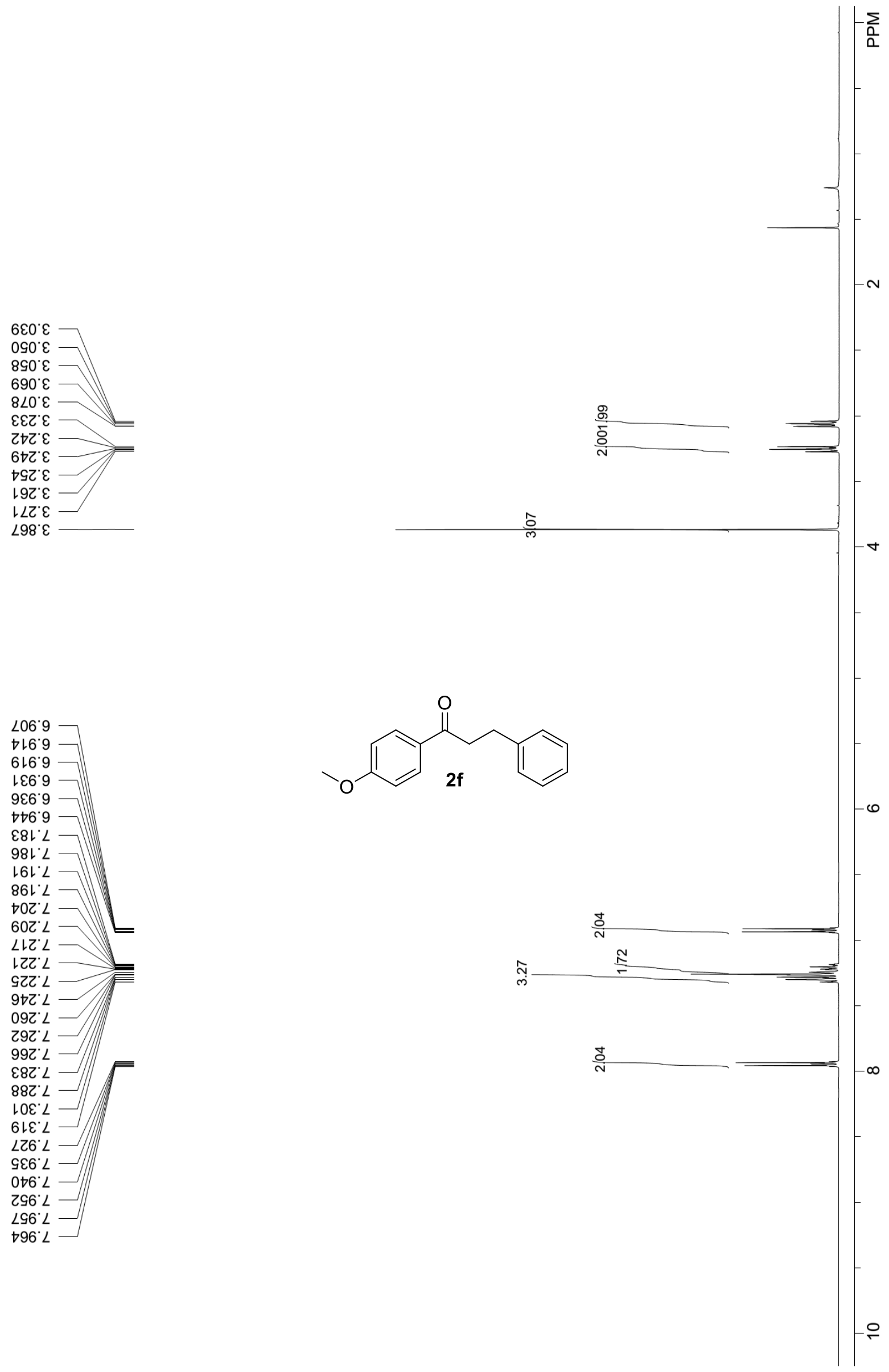


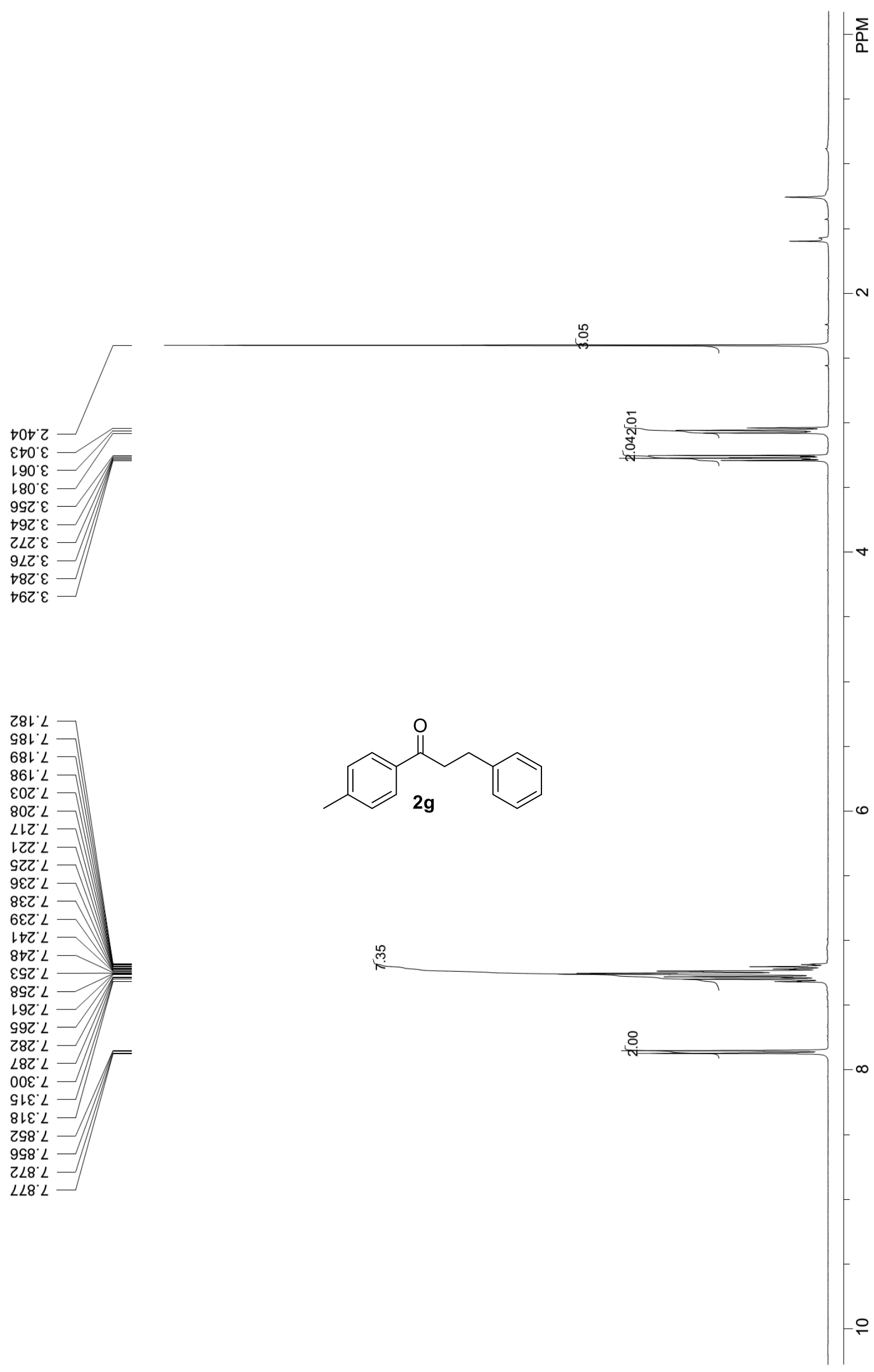




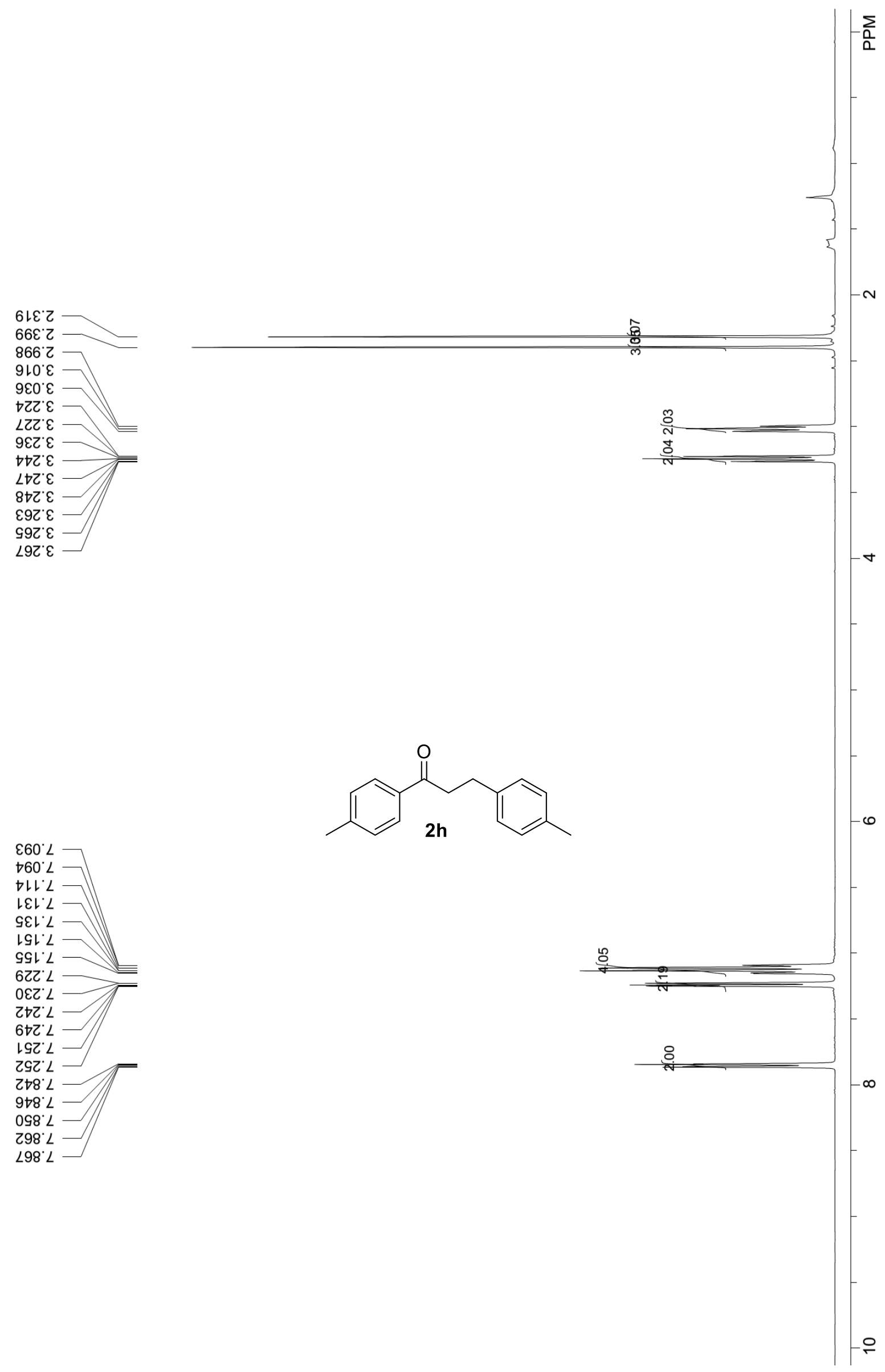




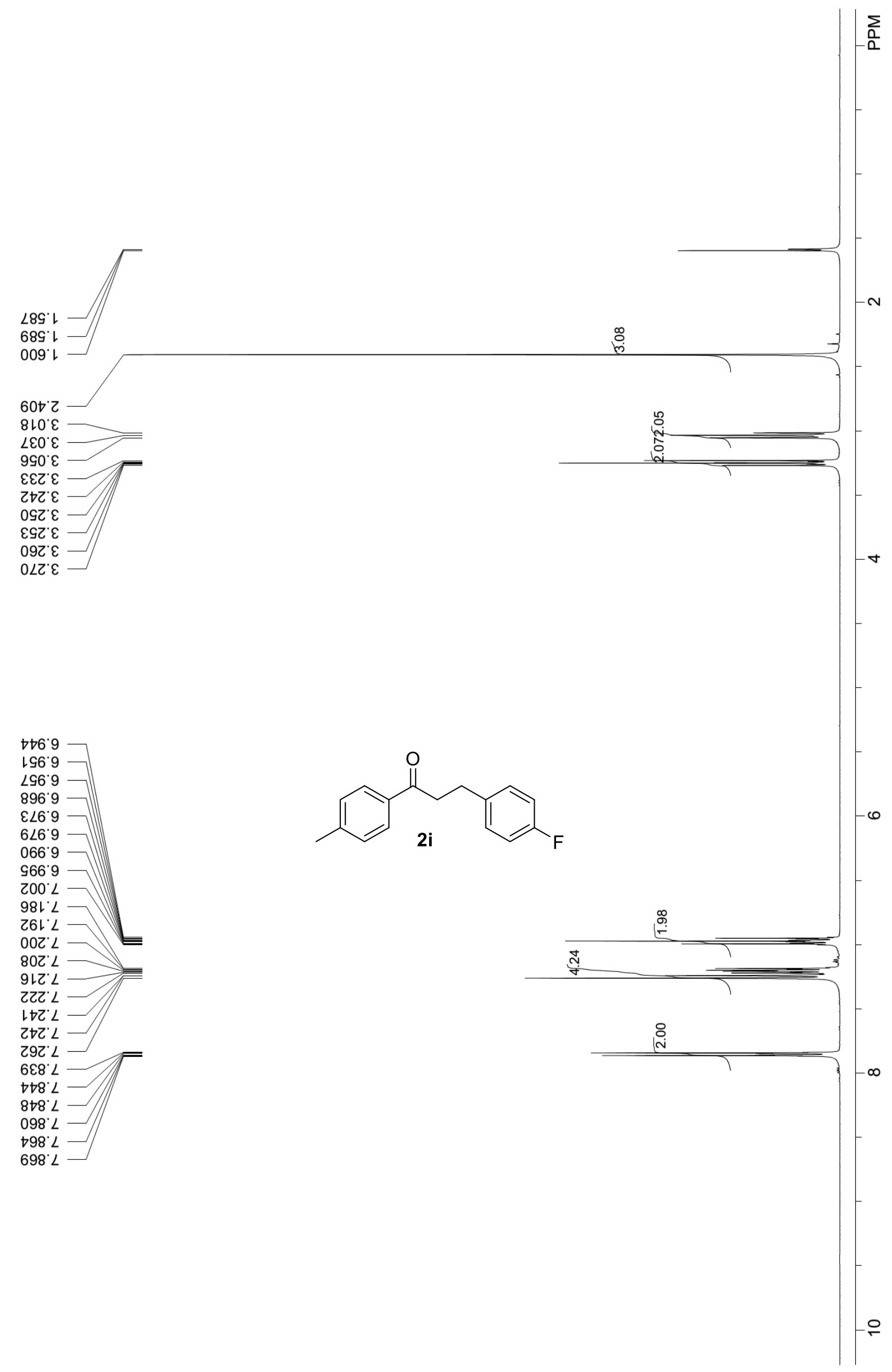


$92 ナ 62$

$8 \angle \varepsilon^{\circ} 0 t$

LLL'9L

$00 L^{\circ} L L$

IVTLL

G6เงเ

60tงเレ

6ZZ'8ZL

乙8६' $62 \mathrm{~L}$

$898^{\circ} 6 \mathrm{ZL}$

t७6 $62 \mathrm{~L}$

เย๋ $ォ \varepsilon L$

$990 . \angle \varepsilon L$

$\neg 60^{\circ} \angle \varepsilon L$

$\angle L O D t$<smiles>Cc1ccc(C(=O)CCc2ccc(F)cc2)cc1</smiles>

५ระ.09L

6 t9. $29 \mathrm{l}$

$\angle 6 L ' 86 L$

2i

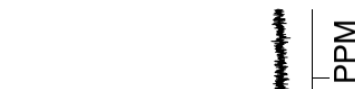

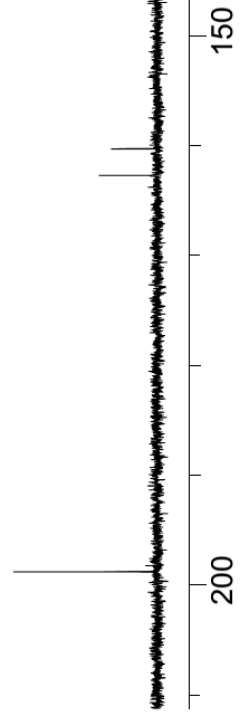

S-67 


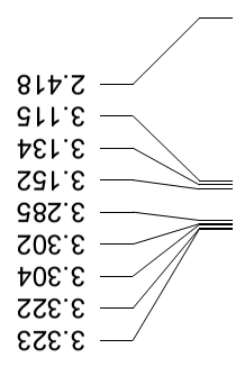

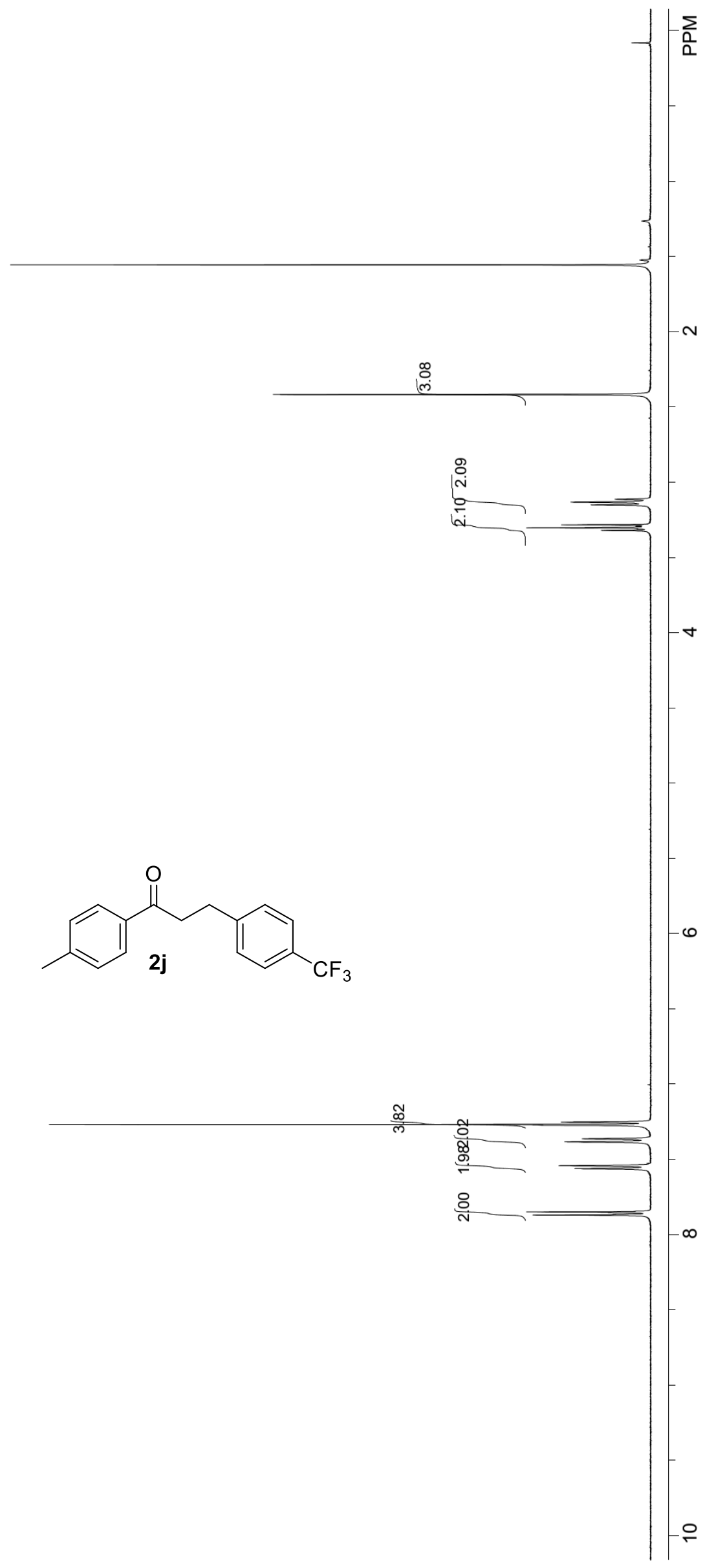

\&Gट L

69Z'L

$\neg 9 \varepsilon^{\circ} L$

$\triangleright 8 \varepsilon^{\circ} L$

$\varepsilon \nabla \mathcal{G}^{\circ}$

E99' $L$

G†8

098.

$\downarrow 98^{\circ}$

$\angle 98^{\circ}$

$1 \angle 8^{\circ} \mathrm{L}$

$9 \angle 8^{\circ} L$ 
$0 \angle 9^{\circ}$ ル

†89 62

ILL6E

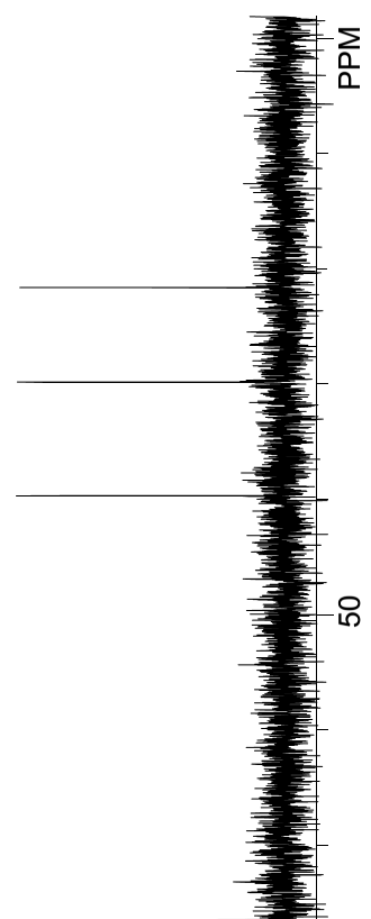

५Et '૬乙।

$980^{\circ} 8 \mathrm{Z}$

6 t9. 82

ISE 62

$980^{\circ} \nabla \varepsilon L$

LLL・DL

$\forall t 9$ 'งt<smiles>Cc1ccc(C(=O)CCc2ccc(C(F)(F)F)cc2)cc1</smiles>

LEะ'86L 


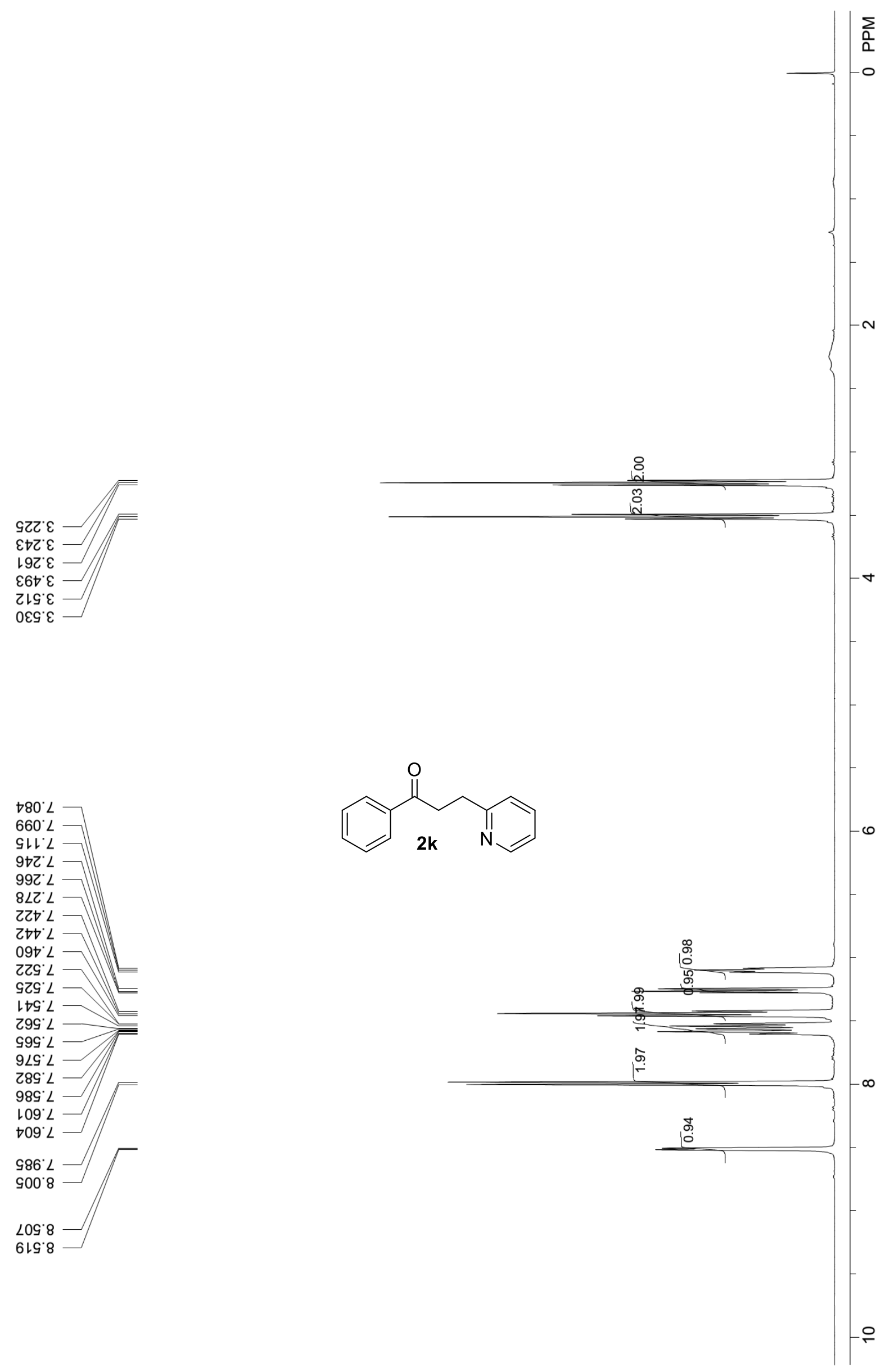




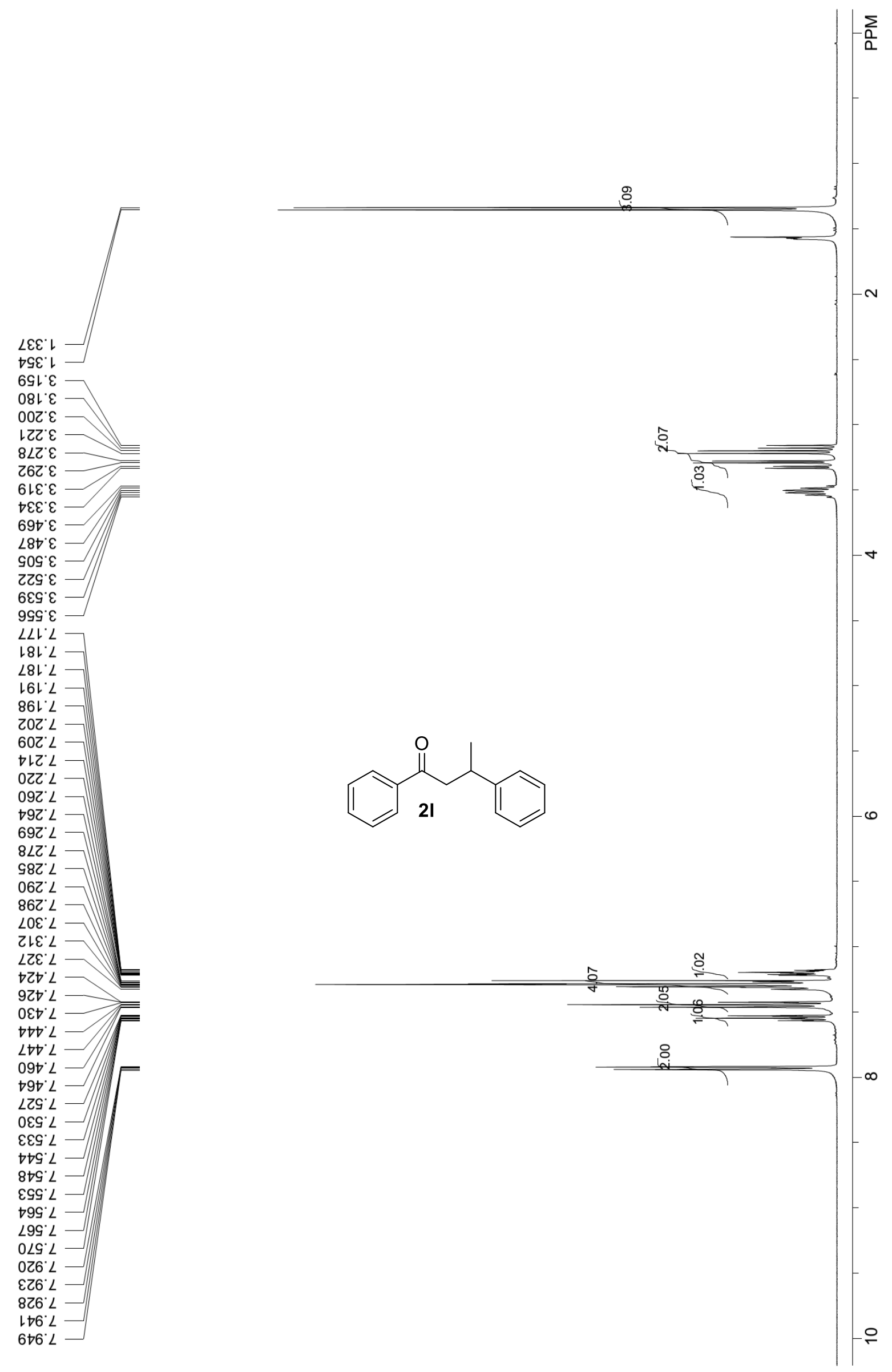



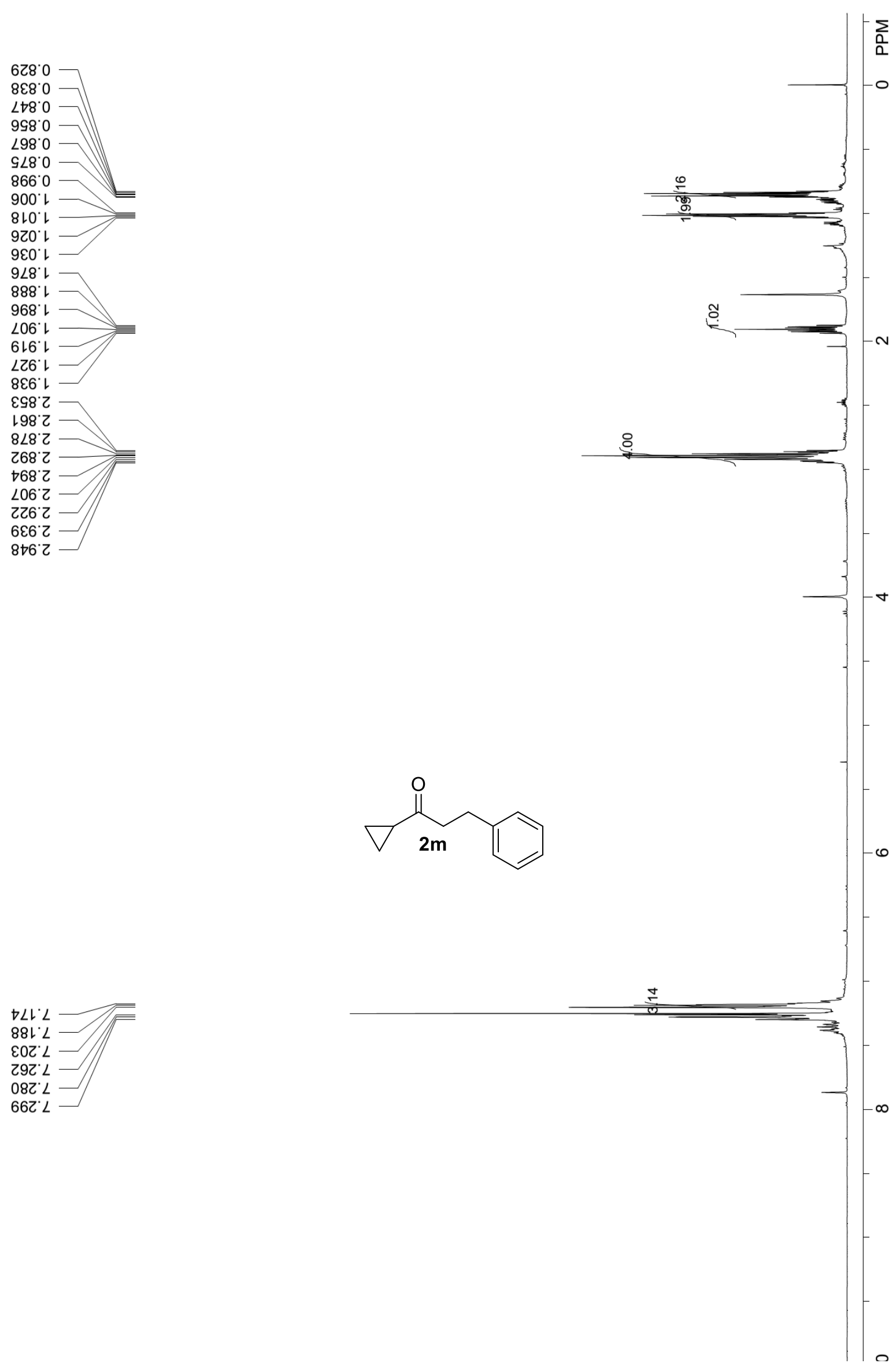

$\checkmark L L L \longrightarrow$

$88 \mathrm{~L} L$

EOZ' $L$

Z9Z'

082

$66 Z^{\circ} \mathrm{L}$

S-72 

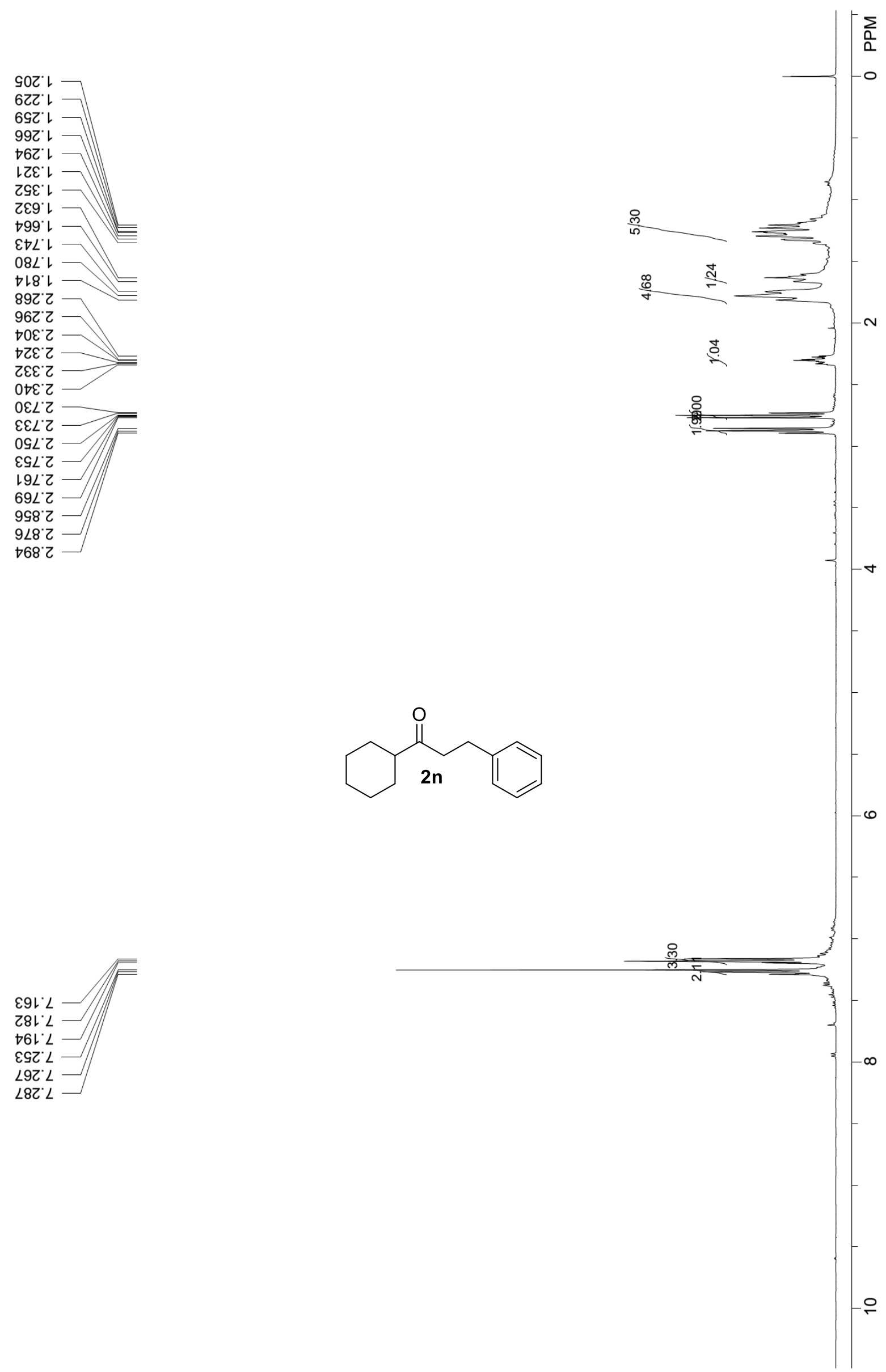

S-73 


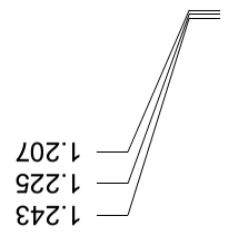

|| \begin{tabular}{l|l}
$-\infty$ \\
$-a$
\end{tabular}
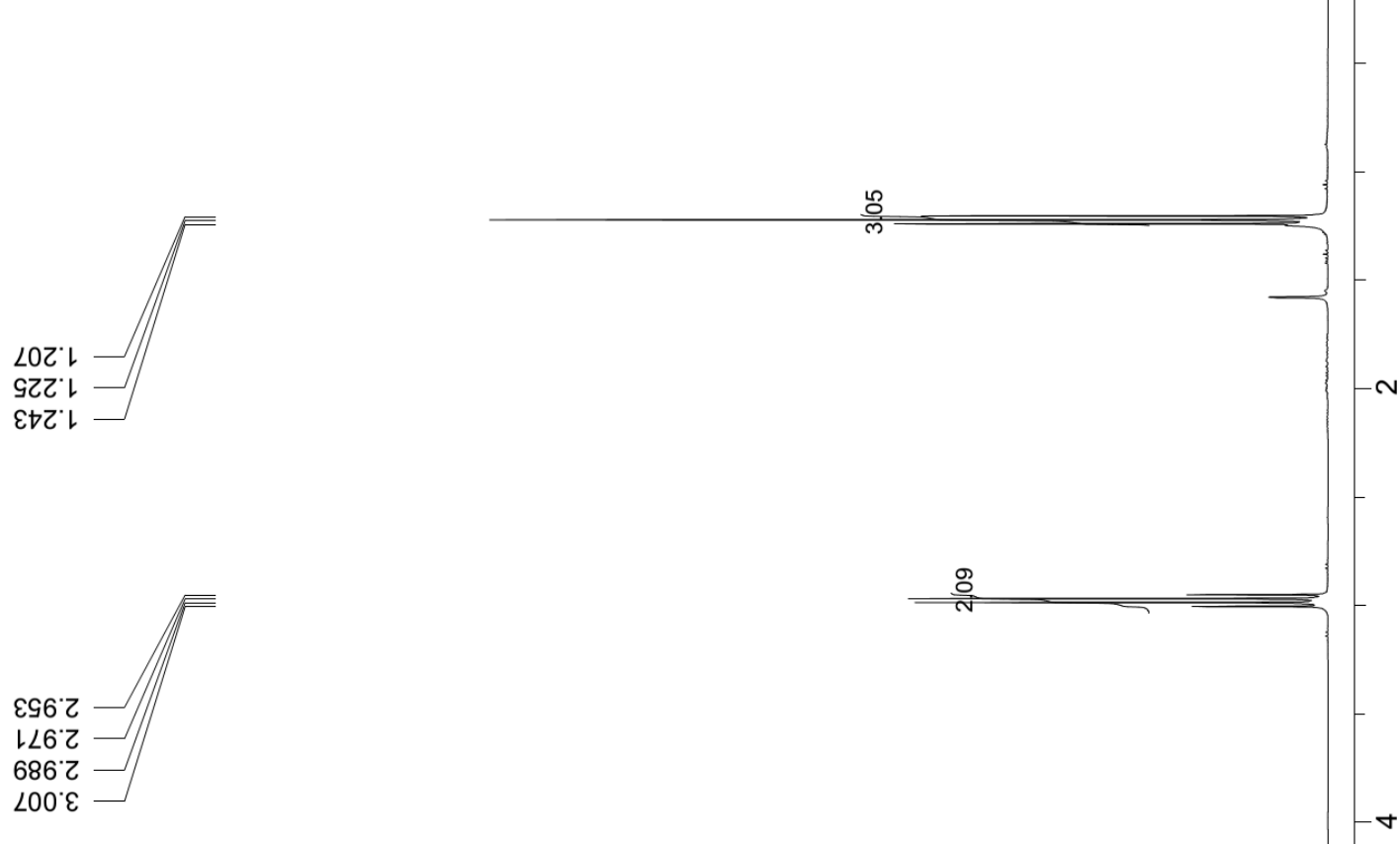

6SZ L

$08 \varepsilon^{\circ} L$

$00 t^{\circ} L$

$0 Z \nabla^{\circ} \mathrm{L}$

$809^{\circ} \mathrm{L}$

OLG'

ELS. $L$

SIG' $L$

$829^{\circ} \mathrm{L}$

IES $L$

EES $L \longrightarrow$

9E9. $L$

$0 Z 8^{\circ} L$
$\varepsilon Z 8^{\circ} L$

¿Z८.

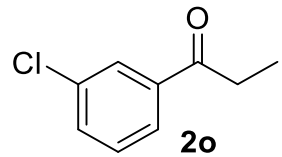

$0 \rightarrow 8$.

乙†8.

$978 . \mathrm{L}$

GZ6.

$626^{\circ} \mathrm{L}$

$\neg \varepsilon 6^{\circ} \angle$

20 
802

†ZZ L

$\nabla \nabla Z$ '

9 เt'

096.2

$8 \angle 6^{\circ} \mathrm{Z}$

$966^{\circ} \mathrm{C}$

$\varepsilon\left\llcorner 0^{\circ} \varepsilon\right.$

OSZ L

$69 Z^{\circ} L$

$798^{\circ} \mathrm{L}$

G88.

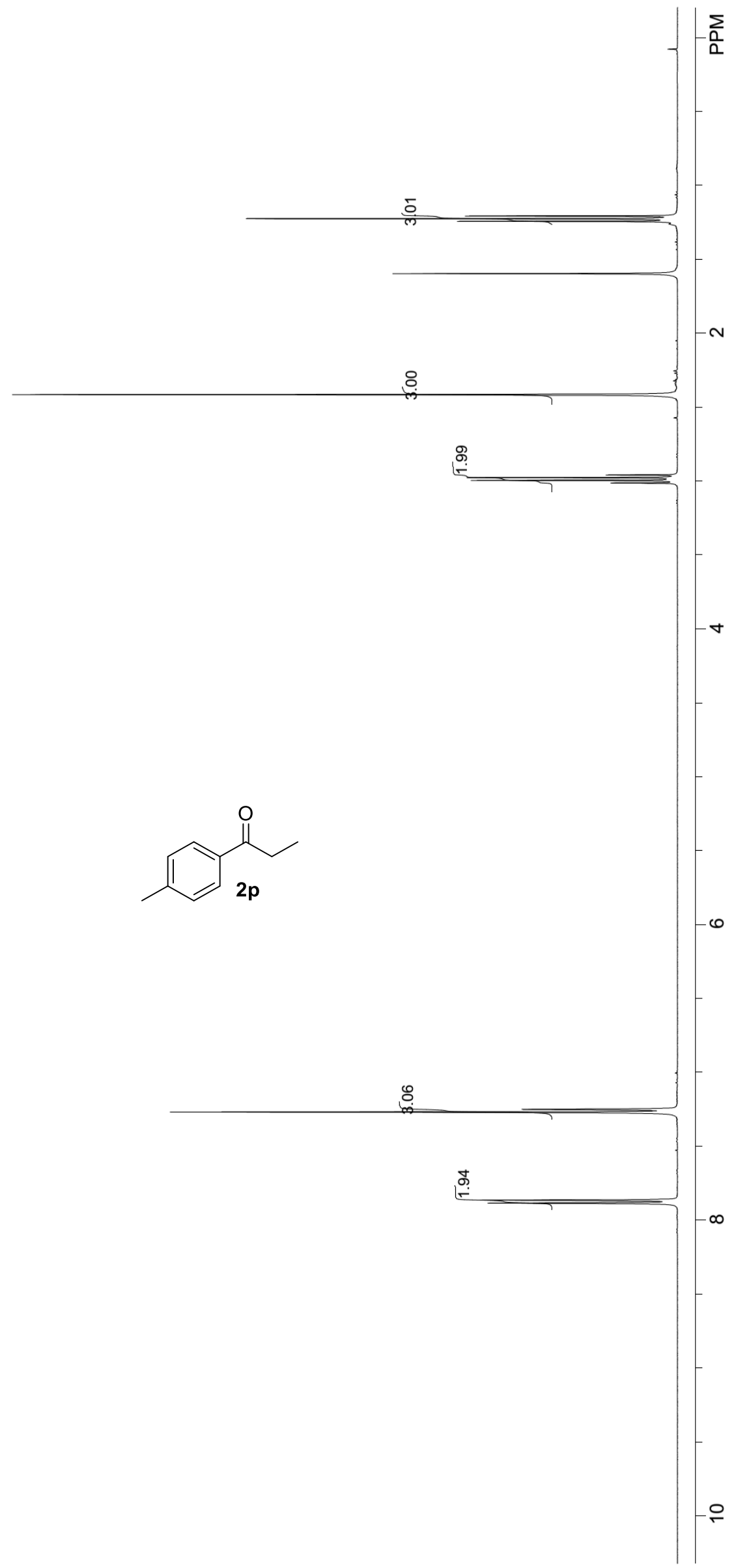

S-75 


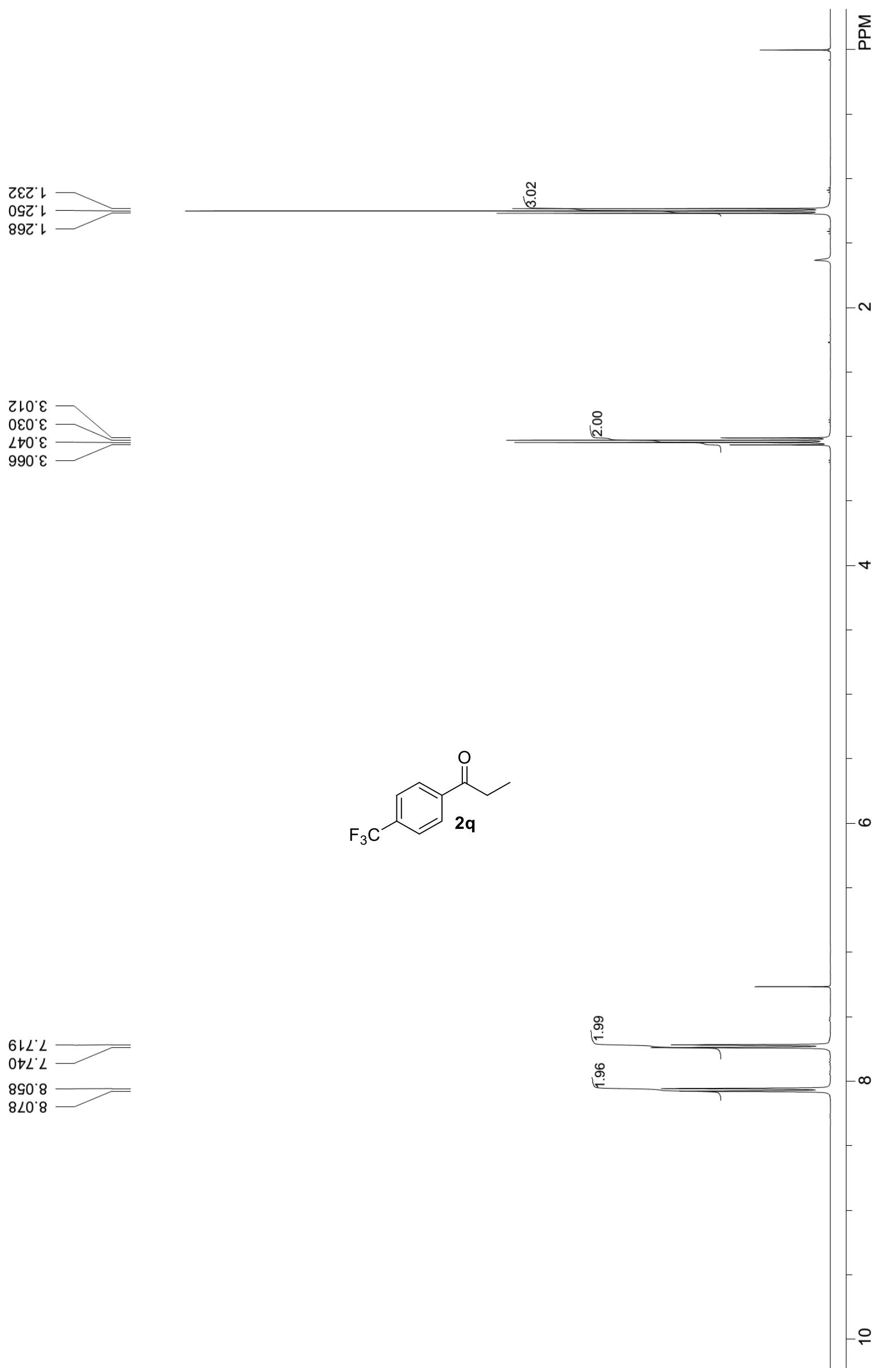

S-76 

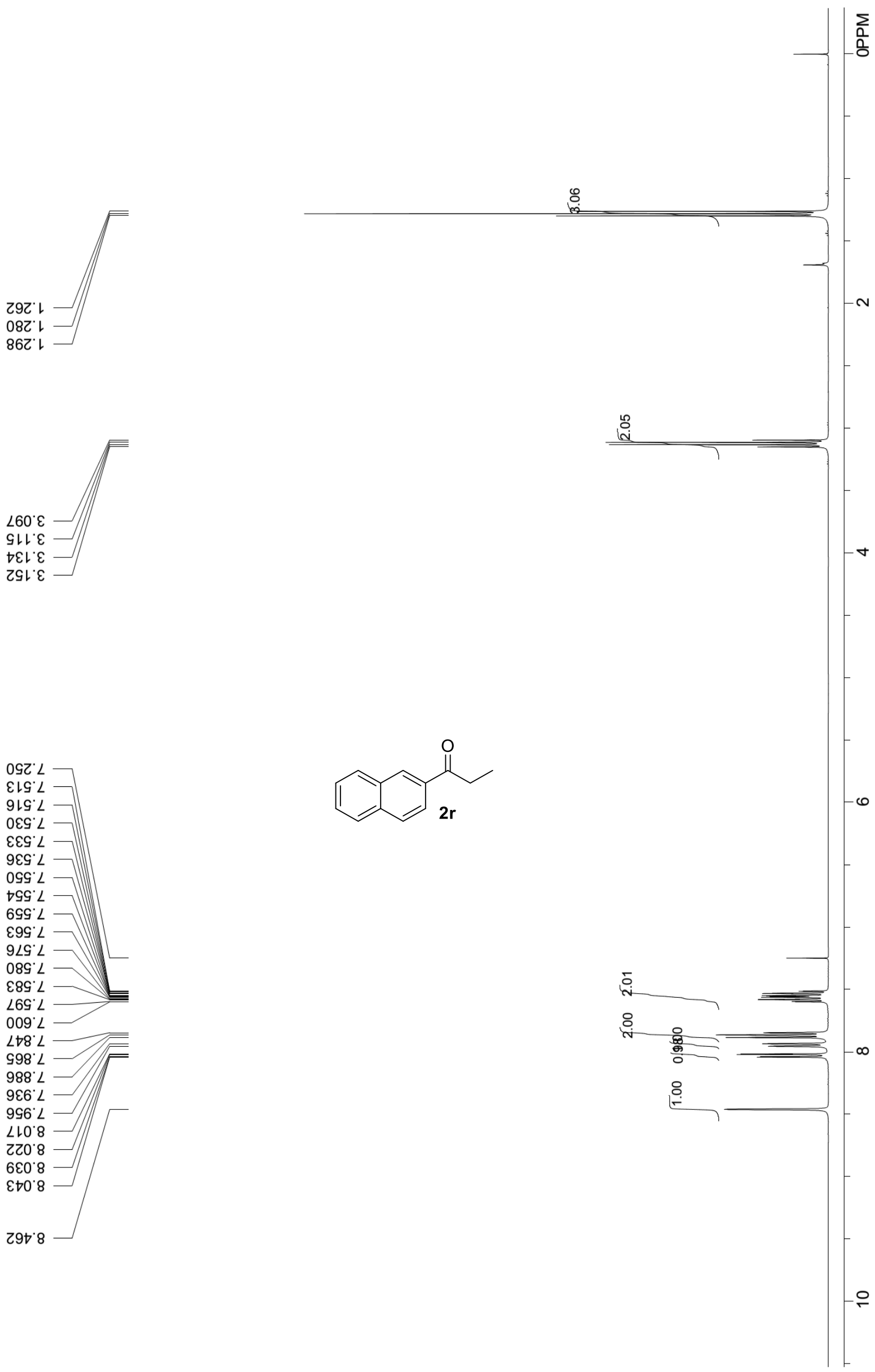


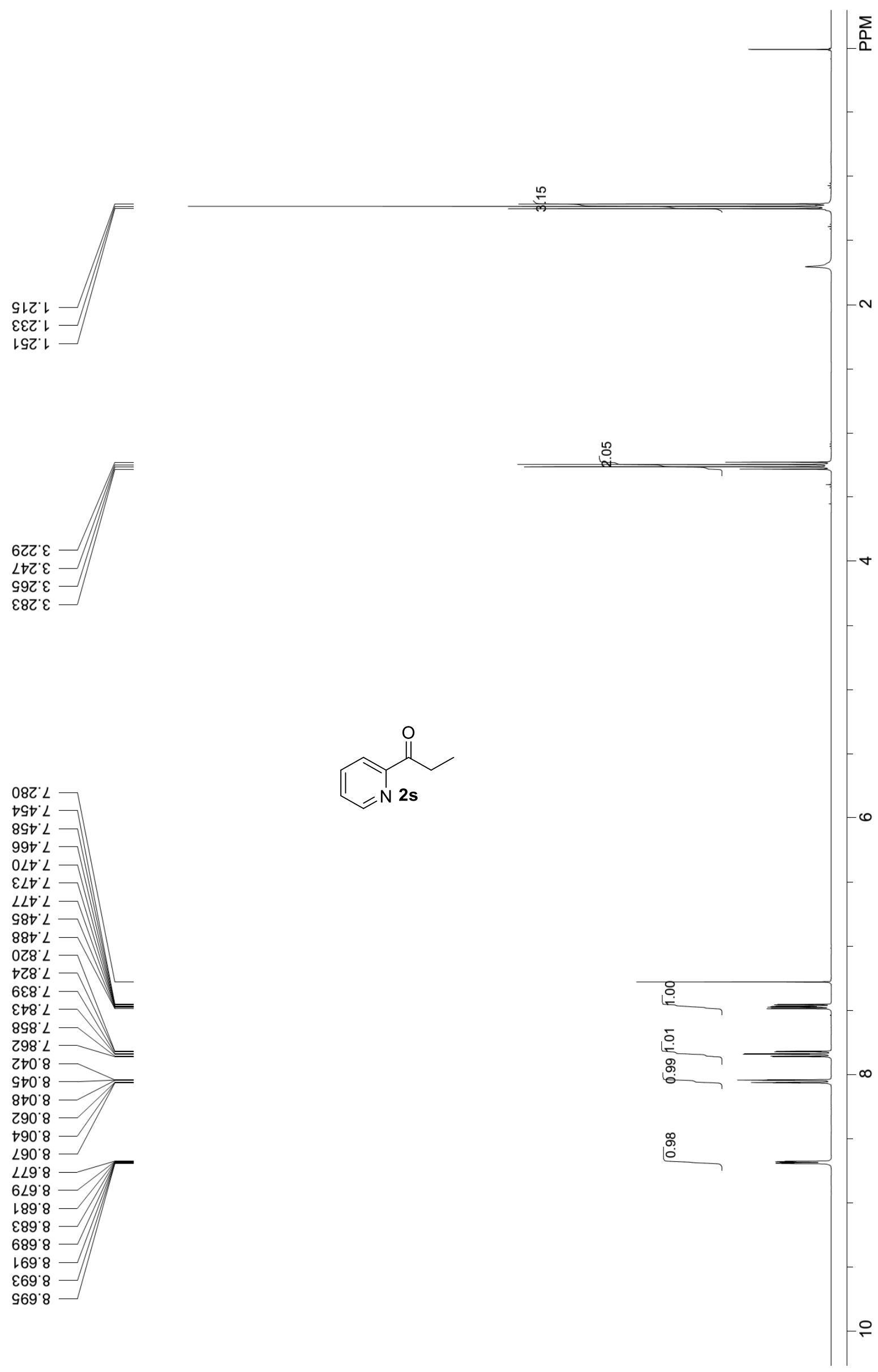

S-78 


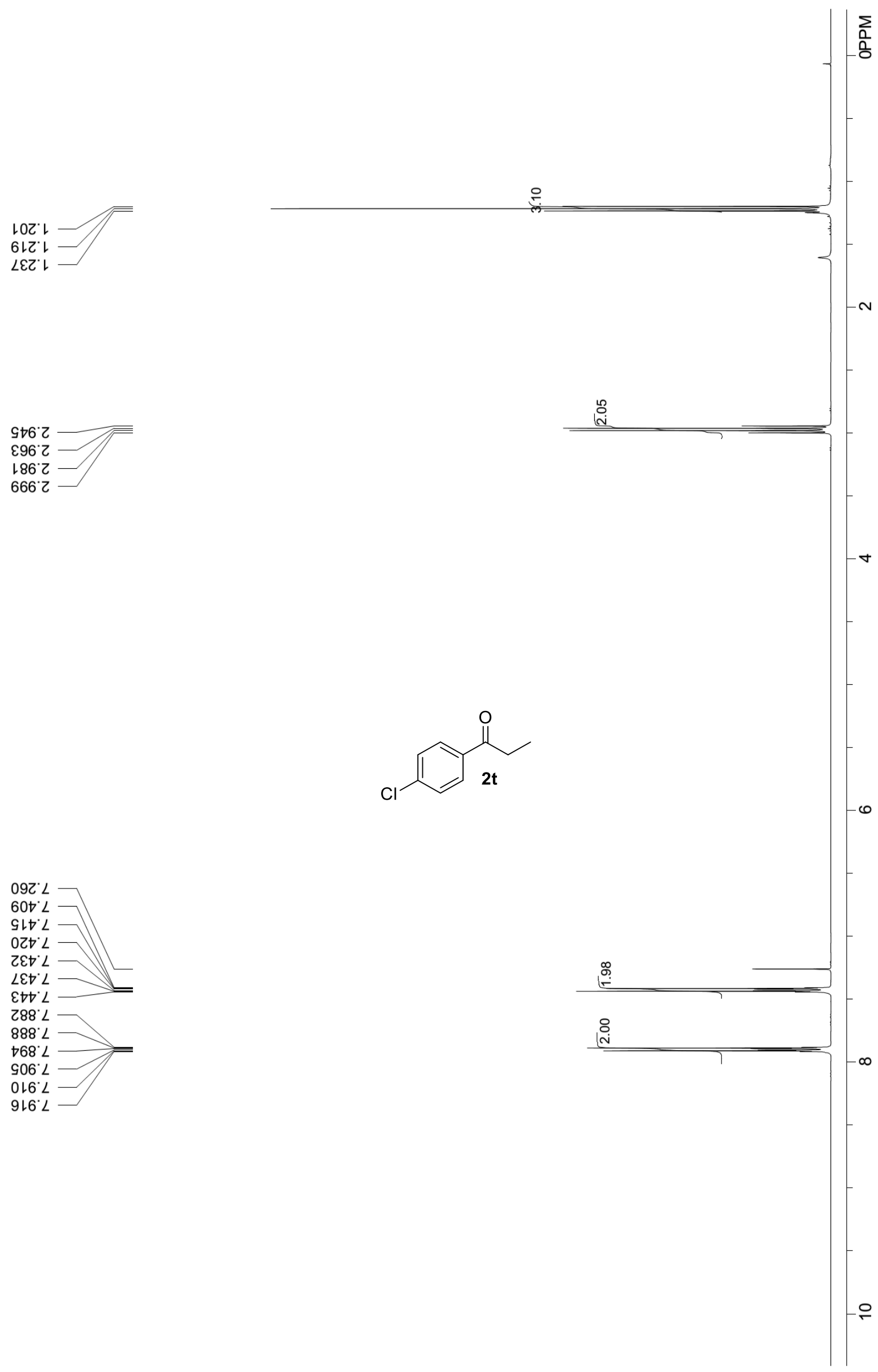



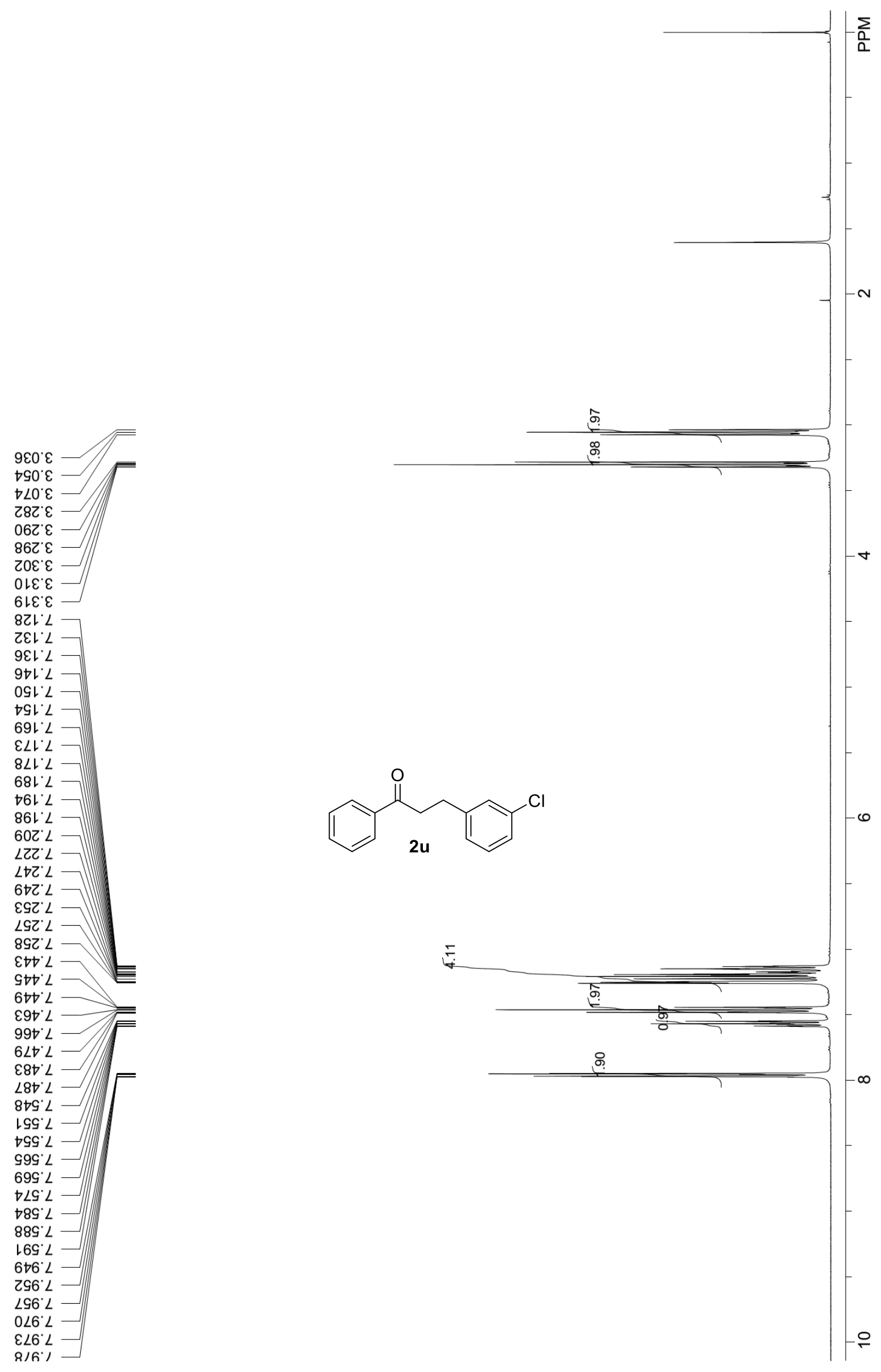


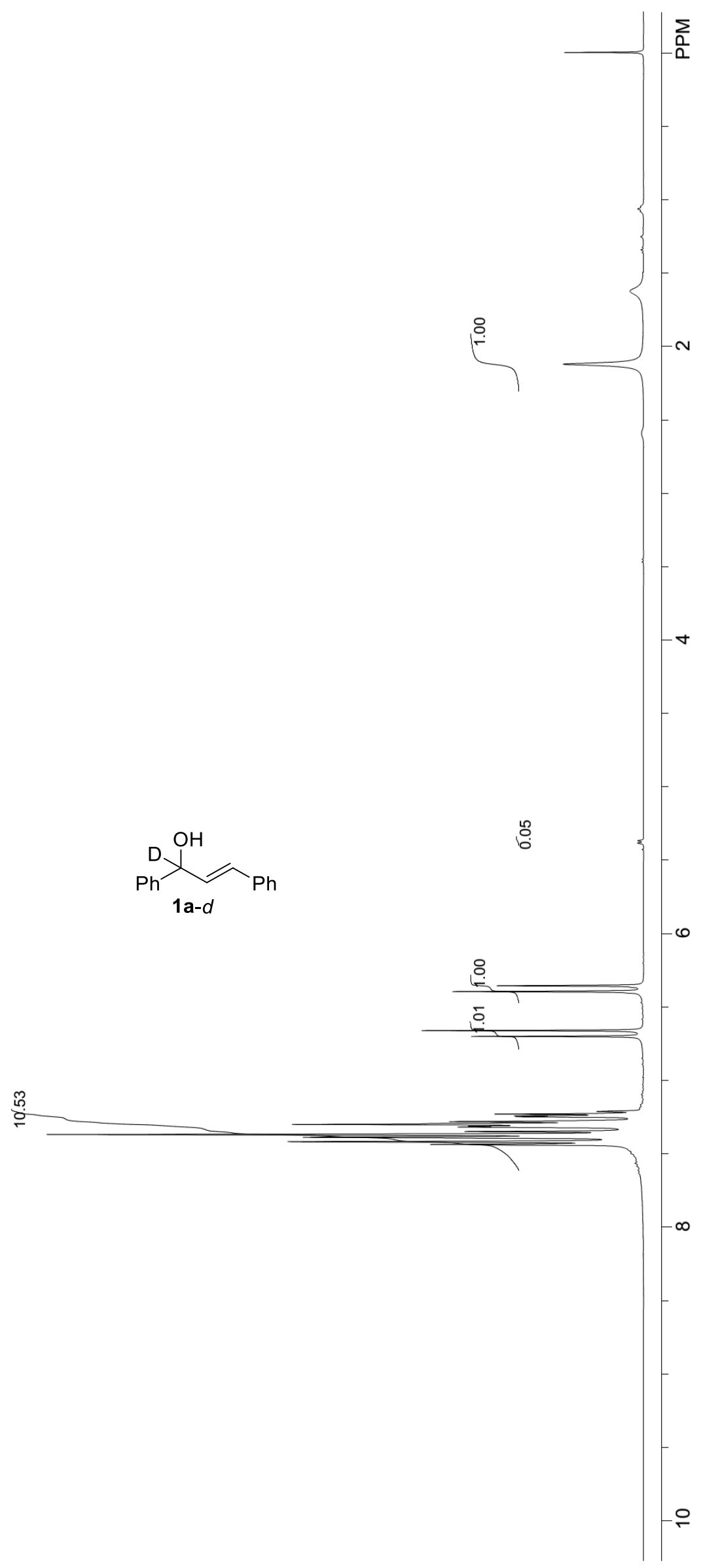

